\title{
Plant communities of moist rock crevices with endemic Primula carniolica in the (sub)montane belt of western Slovenia
}

\author{
Igor Dakskobler ${ }^{1} \&$ Andrej Martinčič ${ }^{2}$
}

Key words: phytosociology, synsystematics, calcareous rock faces, Astrantia carniolica, Pinguicula alpina, Primula carniolica, Saxifraga aizoides, Natura 2000, Julian Alps, Dinaric Alps, Slovenia.

Ključne besede: fitocenologija, sinsistematika, karbonatno skalovje, Astrantia carniolica, Pinguicula alpina, Primula carniolica, Saxifraga aizoides, Natura 2000, Julijske Alpe, Dinarsko gorstvo, Slovenija.
Received: 6. 2. 2020

Revision received: 30. 3. 2020

Accepted: 6. 4. 2020

\begin{abstract}
Based on our analysis of a large number of relevés of communities of moist rock crevices in western Slovenia (southern Julian Alps, northern part of the Trnovski Gozd Plateau) we described several new syntaxa with the endemic Primula carniolica. The most unique in terms of ecology is a hygrophytic chasmophytic community classified into the association Astrantio carniolicaePrimuletum carniolicae, which is, together with similar hygrophytic associations Astrantio carniolicae-Pinguiculetum alpinae and Campanulo cespitosae-Saxifragetum aizoidis, associations Phyteumato columnae-Primuletum carniolicae, Primulo carniolicae-Potentilletum clusianae and the subassociation Primuletum carniolicae violetosum biflorae, temporarily classified into the alliance Cystopteridion and order Potentilletalia caulescentis. Only the new association Paederoto luteae-Potentilletum caulescentis is classified into the alliance Physoplexido comosae-Saxifragion petraeae. The elevational range of chasmophytic communities with Primula carniolica is 200 to $1460 \mathrm{~m}$ a.s.l. Species that most commonly accompany Primula carniolica in rock crevices include Paederota lutea, Phyteuma scheuchzeri subsp. columnae, Sesleria caerulea, Aster bellidiastrum and moss Orthothecium rufescens.
\end{abstract}

Izvleček

Z obdelavo obsežnega števila fitocenoloških popisov združb vlažnih skalnih razpok v zahodni Sloveniji (južne Julijske Alpe, severni del Trnovskega gozda) smo opisali nekatere nove sintaksone, v katerih uspeva endemit Primula carniolica. Ekološko najbolj posebna je vlagoljubna združba skalnih razpok, ki jo uvrščamo v asociacijo Astrantio carniolicae-Primuletum carniolicae. Skupaj s podobnima vlagoljubnima asociacijama Astrantio carniolicae-Pinguiculetum alpinae in Campanulo cespitosae-Saxifragetum aizoidis ter tudi asociacijama Phyteumato columnae-Primuletum carniolicae in Primulo carniolicae-Potentilletum clusianae ter subasociacijo Primuletum carniolicae violetosum biflorae jih za zdaj uvrščamo v zvezo Cystopteridion in red Potentilletalia caulescentis. Le novo asociacijo Paederoto luteae-Potentilletum caulescentis uvrščamo v zvezo Physoplexido comosae-Saxifragion petraeae. Razpon nahajališč združb skalnih razpok z vrsto Primula carniolica je od $200 \mathrm{~m}$ do $1460 \mathrm{~m} \mathrm{~nm}$. v. Vrste, ki v skalnih razpokah najbolj pogosto rastejo skupaj z njo pa so Paederota lutea, Phyteuma scheuchzeri subsp. columnae, Sesleria caerulea, Aster bellidiastrum in mah Orthothecium rufescens.

\footnotetext{
1 Scientific Research Centre of the Slovenian Academy of Sciences and Arts, Institute of Biology, Regional Unit Tolmin, Brunov drevored 13, 5220 Tolmin, Slovenia E-mail: igor.dakskobler@zrc-sazu.si

2 Zaloška 78 a, SI-1000 Ljubljana, Slovenia. E-mail: andrej.martincic@siol.net
} 


\section{Introduction}

Primula carniolica is endemic to Slovenia and has a relatively small distribution area in the pre-Alpine and $\mathrm{Di}$ naric phytogeographical region of Slovenia, which spans the foothills of the southern Julian Alps and NW Dinaric Alps. Its northernmost localities are in the northeastern edge of the Šentviška Gora Plateau at the village Bukovski Vrh, in the gorge Selska Voda under the village of Bukovo and in Orehovska Grapa gorge under the hamlet of Nemci at the foothills of Mt. Kojca. The southeasternmost locality is under Pšeničev Vrh above Sodražica and the ridge of Velika Gora above Ribnica (Figure 1, see also Dakskobler et al. 2004, Dolinar et al. 2013, Dakskobler \& Vreš 2014). There are many localities in its distribution area and they frequently feature many specimens. The Carniolan primrose occurs in different plant communities, but mainly on dolomite or dolomite-limestone bedrock, most often on rocky and at least slightly moist sites, in narrow, hard-to-access ravines, more rarely on karstified Dinaric plateaus, at elevations spanning 200 to $1460 \mathrm{~m}$. Numerous plant communities featuring this species have already been supported by phytosociological tables. It has been identified (by the authors of this study and others) in stands of the following forest syntaxa: $\mathrm{Om}$ phalodo-Fagetum rhododendretosum hirsuti (Dakskobler et al. 2000, Surina \& Dakskobler 2013), Rhododendro hirsuti-Fagetum (Dakskobler 2003, Accetto 2015), AruncoFagetum (Dakskobler 2015a), Polysticho lonchitis-Fagetum (Dakskobler \& Rozman 2020, in preparation), Lamio orvalae-Aceretum pseudoplatani (Dakskobler 2007), Lamio orvalae-Salicetum eleagni caricetosum albae (Dakskobler 2010), Fraxino orni-Ostryetum phyteumatetosum columnae (Dakskobler 2015b), Rhododendro hirsuti-Ostryetum (Dakskobler 2015b, Accetto 2015), Ostryo carpinifoliaePiceetum (Accetto (2013), Fraxino orni-Pinetum nigrae var. geogr. Primula carniolica (Dakskobler 1998, 1999, Accetto 2015), Primulo carniolicae-Pinetum nigrae (Accetto 2008, 2015), Genisto januensis-Pinetum sylvestris campanuletosum cespitosae (Rozman et al. 2020). It has been recorded in several grassland communities as well: Primulo carniolicae-Caricetum firmae, Primulo carniolicae-Seslerietum calcariae (Surina \& Dakskobler 2005, Dakskobler 2006), Saxifrago squarrosae-Caricetum mucronatae var. geogr. Primula carniolica (Dakskobler 2006, Dakskobler \& Surina 2017a), Primulo carniolicae-Caricetum sempervirentis (Accetto 2010), in a frost pocket community of moist rocky hollows Drepanoclado uncinati-Heliospermetum pusillae (Surina \& Vreš 2009), in a community of moist screes Astrantio carniolicae-Adenostyletum glabrae (Dakskobler 2008), community of dolomite headwaters Astrantio carniolicae-Schoenetum nigricantis (Dakskobler
\& Martinčič 2018) and in two shrub communities of rocky sites, namely Heliospermo pusillae-Rhododendretum hirsuti and Rhododendro hirsuti-Salicetum appendiculatae (Dakskobler \& Surina 2017b).

In most of the listed forest and grassland communities Primula carniolica occurs with a lower frequency and cover than in chasmophytic (rock-face) communities, where its optimal sites are. Only two such communities have been described to date: Primulo carniolicae-Potentilletum caulescentis (Dakskobler 1998, Accetto 2008) and Primuletum carniolicae (Accetto 2008, 2015, Dolinar et al. 2013, 2015, 2017). Most of our relevés of chasmophytic communities with Primula carniolica have not yet been processed and published. In this article we provide a brief description of the identified communities and try to find an appropriate syntaxonomic classification for some of them.

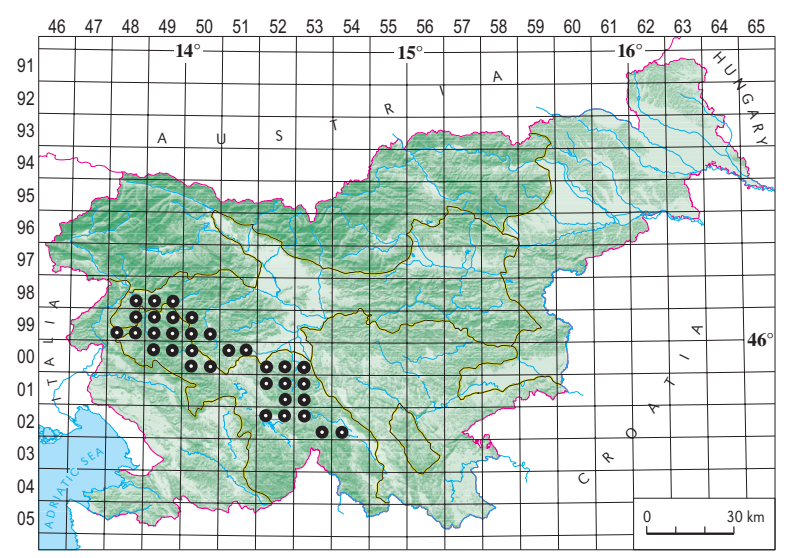

Figure 1: Distribution of Primula carniolica in Slovenia (FloVegSi database).

Slika 1: Razširjenost vrste Primula carniolica v Sloveniji (podatkovna baza FloVegSi).

\section{Methods}

Our analysis was based on the phytosociological relevés recorded on the sites of Primula carniolica using the Central-European (Braun-Blanquet 1964) approach. The relevés were made in the northwestern part of its distribution area, between Čepovan, Slap ob Idrijci, Bukovski Vrh, Bukovo, Orehek in the northwest and north, and the Golaki range, Črni Vrh nad Idrijo and the Žirovnica valley near Žiri in the south and southeast. The plot size was determined based on site homogeneity. Relevés were entered into the FloVegSi database (Fauna, Flora, Vegetation and Paleovegetation of Slovenia) of the Jovan Hadži Institute of Biology at ZRC SAZU (Seliškar et al. 2003). In further analyses we focused only on the relevés of chasmophytic communities in this area (altogether about 320 - Figure 2) and ordered them using hierarchical classifica- 
tion, unweighted average linkage method - UPGMA and Wishart's similarity ratio, using SYN-TAX 2000 program package (Podani 2001). We transformed the combined cover-abundance values with numerical values (1-9) according to van der Maarel (1979). The UPGMA method was used because it better recognises the relevés that stand out from others. The results of the UPGMA correspond to our experience in the field, as the clusters comprise the relevés (stands) that are in fact ecologically similar.

The relevés where Primula carniolica is mostly accompanied by distinctly hygrophytic species Pinguicula al- pina, Astrantia carniolica, Hymenostylium recurvirostre and Palustriella commutata (the smaller group) clustered separately from all other relevés (the bigger group), which either comprised only some of the above-listed species, usually with low mean coverage, or none of these species (Dendrogram, Figure 14 in Appendix). In the next step, these two, distinctly different groups of relevés were processed separately, but without taking into account all similar relevés in the bigger group, in order to avoid the tables being too extensive.

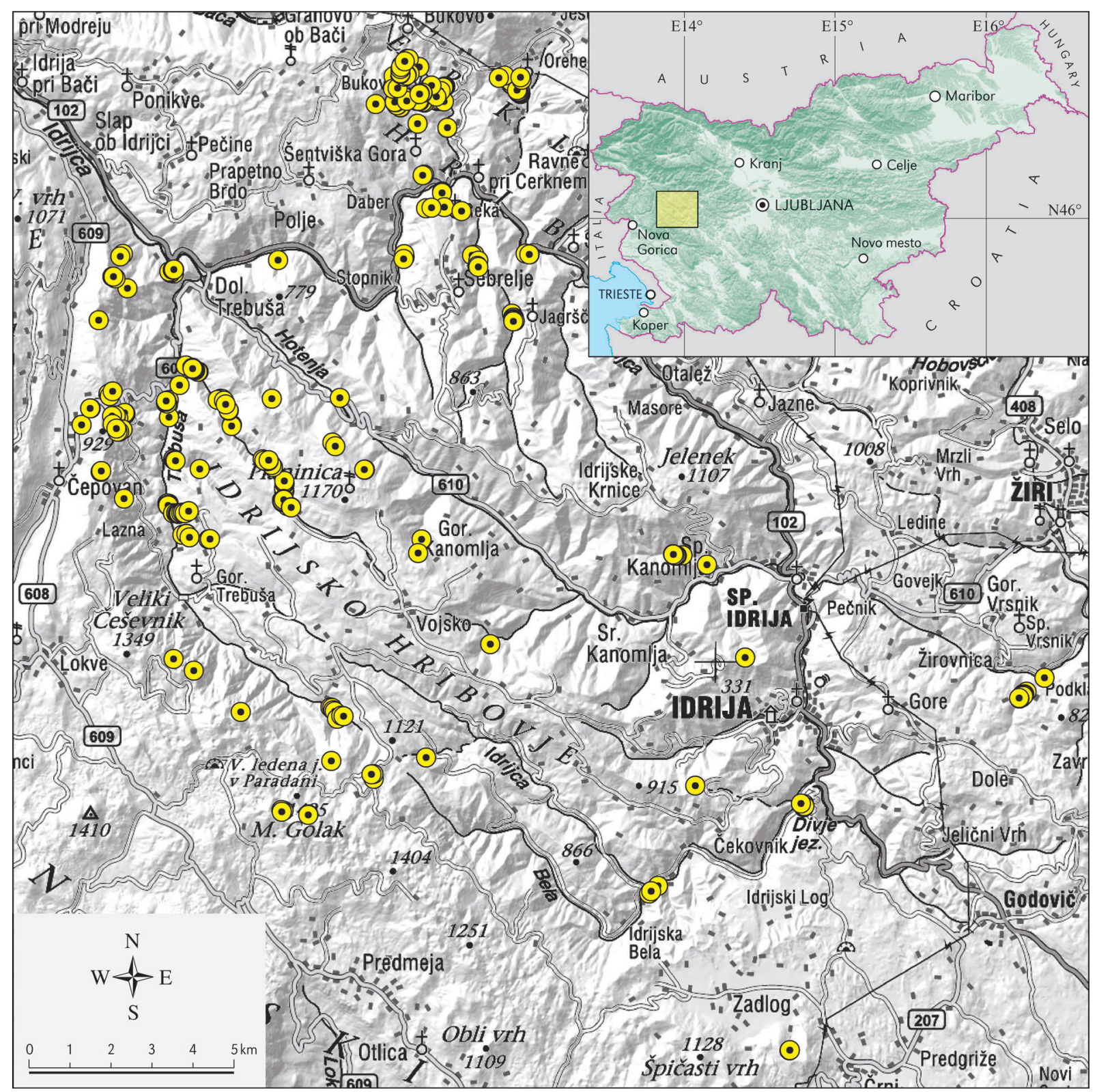

Figure 2: Localities of rock crevice communities with Primula carniolica in western Slovenia.

Slika 2: Nahajališča združb skalnih razpok z vrsto Primula carniolica v zahodni Sloveniji. 
For the sake of comparison our analysis included also several other communities of moist rock crevices in the submontane-montane belt of the southern Julian Alps and the northern part of the Dinaric Alps, which are dominated by Pinguicula alpina and (or) Saxifraga aizoides, have a rich moss layer, but do not comprise Primula carniolica (these communities are partly distributed outside its distribution area). These relevés were arranged into tables in the same manner, through hierarchical classification, the same as the columns in the synoptic table. Numerical comparisons were performed with the SYN-TAX 2000 program package (Podani 2001). When describing new associations of plant communities with poor species composition and few dominant species, we focused on the latter and their mean coverage, rather than only on floristic similarity between the compared syntaxa.

The nomenclatural source for the names of vascular plants was the Mala flora Slovenije (Martinčič et al. 2007), except for the name Molinia arundinacea Schrank. The nomenclature of Flora alpina (Aeschimann et al. 2004) - Sesleria caerulea was used for the taxon Sesleria caerulea subsp. calcaria (MFS). Ros et al. (2007) was the nomenclatural source for the names of liverworts (Marchanthiophyta) and Ros et al. (2013) for the names of mosses. Šilc \& Čarni (2012), Accetto (2015), Mucina et al. (2016), Dakskobler \& Surina (2017a, b) and Dakskobler \& Martinčič (2018) served as nomenclatural sources for the names of the syntaxa. The data source for geological bedrock was Buser (2009). The geographic coordinates of the relevés were determined according to the Slovenian geographic coordinate system D 48 (Zone 5) on the Bessel ellipsoid and with Gauss-Krüger projection.

\section{Results and discussion}

\section{Arrangement of relevés into tables}

Our analysis of 348 relevés of rock crevices with Primula carniolica (from its entire distribution area), showed that even though they comprise about 300 species of vascular plants and mosses, the species with constancy higher than 50\% are (in addition to Primula carniolica) only Paederota lutea, Phyteuma scheuchzeri subsp. columnae, Sesleria caerulea, Aster bellidiastrum and moss Orthothecium rufescens. Seven species have the constancy of $40 \%$ or higher: Astrantia carniolica, Asplenium ruta-muraria, Valeriana saxatilis, Asplenium trichomanes, Calamagrostis varia and mosses Neckera crispa and Ctenidium molluscum. The entire species composition of these communities features less than $10 \%$ of the species with constancy higher than
$20 \%$ and only 42 species ( 35 vascular plants and 7 mosses) with constancy more than $10 \%$ (the list of the most common species in chasmophytic communities with Primula carniolica is in Table 11). This clearly demonstrates that the decisive factor when classifying communities with the average number of only 15 to 20 species per relevé into a syntaxonomic system are in the first place the species with constancy of at least $40 \%$ or higher. Some species with the constancy of 10 to $35 \%$, but high mean coverage can be in the first place differential species or species that indicate entirely different sites.

The relevés of distinctly moist calcareous rock crevices, with occasional tufa formations, were arranged into two tables. Table 1 comprises hygrophilous communities where Primula carniolica has similar constancy and mean coverage as Pinguicula alpina and Astrantia carniolica, while Hymenostylium recurvirostre in Palustriella commutata stand out in the moss layer, both with their frequency and mean coverage.

With hierarchical clustering we arranged the stands that were the most similar to these in terms of site moisture (stands with dominating Pinguicula alpina and Astrantia carniolica, with some stands comprising also Saxifraga aizoides) in Table 2 and Table 3 (stands with dominating Saxifraga aizoides).

Table 5 comprises the relevés with three dominant species alongside Primula carniolica: Orthothecium rufescens, Paederota lutea and Phyteuma scheuchzeri subsp. columnae. The latter subspecies is not included in the phytosociological table of the original description of the association Primuletum carniolicae (Accetto 2008), because its distribution area (Figure 3) does not overlap with the entire distribution area of Primula carniolica, but only with its western part, primarily the Idrijca river basin with the Trnovski Gozd Plateau. Ecology of Phyteuma columnae is slightly different from the ecology of Primula carniolica. As it requires less moisture it is more common in very dry rock crevices. It is a character species of the association Phyteumato-Potentilletum caulescentis, whose stands dominate on the southern rocky edge of the Trnovski Gozd and Nanos plateaus (Poldini 1978), more rarely also on their northern side, and of the association Phyteumato-Moehringietum villosae from the foothills of the southern Julian Alps (Dakskobler 2000). In stands with Primula carniolica it is therefore above all a phytogeographical differential species. The entire species composition of the community in Table 5 is not considerably different from the floristic composition of the community in Table 1, but there are obvious differences in terms of constancy and mean coverage of the most frequent species, and in the percentage of groups of diagnostic species (Table 10). 


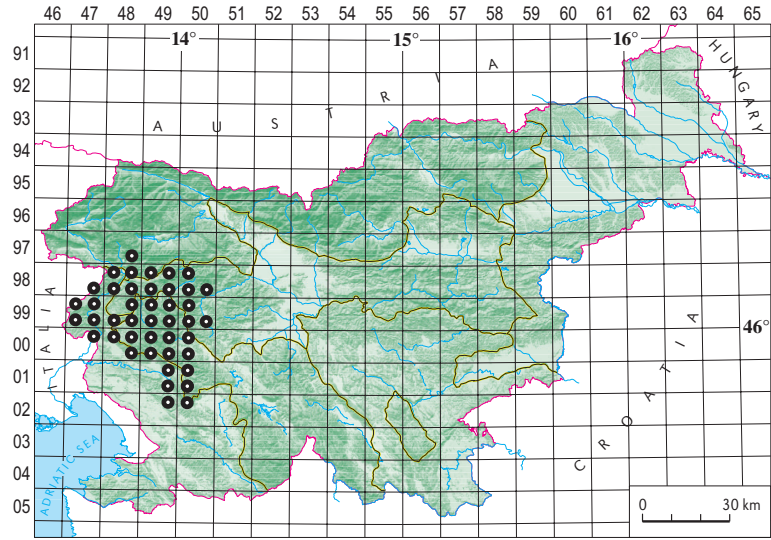

Figure 3: Distribution of Phyteuma scheuchzeri subsp. columnae in Slovenia (FloVegSi database).

Slika 3: Razširjenost taksona Phyteuma scheuchzeri subsp. columnae v Sloveniji (podatkovna baza FloVegSi).

While the relevés arranged in Table 6 are similar to the relevés in Table 5, many of them were made at higher elevations and have a higher frequency of more hygrophytic species such as Astrantia carniolica and Viola biflora. In terms of ecological conditions these relevés indicate a certain similarity also with the community in Table 1 (compare structure by groups of diagnostic species, Table 10).

Table 7 comprises the relevés that grouped separately in the comparison of the relevés of the association Primuletum carniolicae s. lat. and have until now been classified into the association Primulo carniolicae-Potentilletum caulescentis.

Table 8 comprises six relevés from Govci (rock faces under the top of Zeleni Rob and Poldanovec on the northern edge of the Trnovski Gozd Plateau above the Trebuša valley), characterised by Potentilla clusiana, Carex firma, Rhodothamnus chamaecistus and Campanula cochleariifolia.

The relevés in Table 4 grouped separately and were mainly made on the fringe of the distribution area of Primula carniolica, in the gorge Raskovec and the Železnica valley (municipality of Idrija, although historically this territory belongs to Žiri and Gorenjska regions). Viola biflora and Pinguicula alpina are frequent here, but Paederota lutea and Phyteuma scheuchzeri subsp. columnae are very rare.

\section{Syntaxonomic classification of of recorded communities based on the synoptic table}

Table 9 was decisive in our assessment and synsystematic classification of the studied communities. The synoptic table was used because the syntaxa were described with more than one relevé. As no single relevé is fully representative of a syntaxon it is only in a synthetic form (a cluster of relevés) that the author can convey how he understands a certain syntaxon. Individual stands (communities) that occur in nature and are depicted by our relevés can never fully correspond to what the author recognised as a (specific) abstract syntaxon. We therefore compared the syntaxa described in this article, which were obtained with the cluster analysis of individual relevés (see the dendrogram, Figure 14 in the Appendix), with other, already published syntaxa, which were considered in a synthetic form. In addition to most of the communities in the listed tables (except the community in Table 3, which is obviously different from other chasmophytic communities with dominant Pinguicula alpina and/or Primula carniolica) it comprises, in synoptic form, also the following, already published communities: four tables of the association Primuletum carniolicae s. lat. (Dolinar et al. 2013, 2015, 2017) and two published tables of the same association (Accetto 2008, 2015). These six synoptic columns present chasmophytic communities in the central and southeastern part of the distribution area of Primula carniolica (the above-mentioned tables have no relevés from this area). We also added two other forms of the association Primulo carniolicae-Potentilletum caulescentis (Dakskobler 1998, Accetto 2008) to the synoptic table. The table comprises 15 syntaxa. By comparing them we obtained the dendrogram in Figure 4, in which we took into account the species' constancy, and not only their presence or absence. This decision is based on the already mentioned observation that the species that play the decisive role in these communities, which are distributed across small areas and have few recorded species, are in the first place the species with higher constancy and mean coverage. Some researchers assess mean coverage also with a specific cover index (for communities in the territory of Slovenia comp. Surina 2005).

Based on the synoptic table and hierarchical classification we can describe two new associations, Astrantio carniolicae-Primuletum carniolicae (Table 1) and Astrantio carniolicae-Pinguiculetum alpinae (Table 2) whose stands occur on apparently moister sites than those of the communities from previously described associations Primuletum carniolicae and Primulo carniolicae-Potentilletum caulescentis. They are similar also in terms of constancy of diagnostic species, except that the stands of the first association differ so significantly from the stands of the other in terms of certain community-defining species (see also Table 10) that we find the classification of the community in Table 1 into the subassociation Astrantio-Pinguiculetum alpinae primuletosum carniolicae less appropriate.

In terms of entire species composition the stands of the association Astrantio-Pinguiculetum alpinae are slightly similar to the stands of the association Hymenostylio recur- 


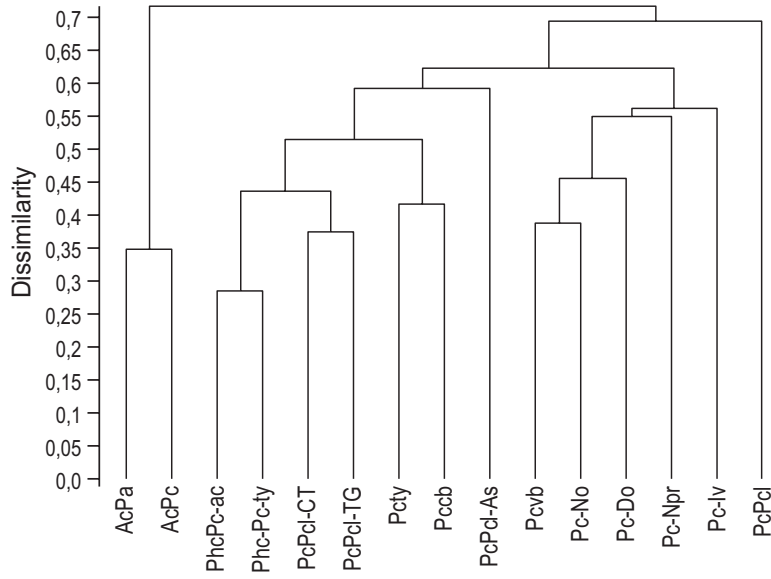

Figure 4: Dendrogram of rock crevices communities with endemic Primula carniolica, UPGMA, 1-similarity ratio.

Slika 4: Dendrogram združb skalnih razpok z endemitom Primula carniolica (UPGMA, 1-similarity ratio).

\section{Legend / Legenda:}

AcPa Astrantio carniolicae-Pinguiculetum alpinae, this article, Table 2.

AcPc Astrantio carniolicae-Primuletum carniolicae, this article, Table 1.

PhcPc-ac Phyteumato columnae-Primuletum carniolicae astrantietosum carniolicae, this article, Table 6.

Phc-Pc-ty Phyteumato carniolicae-Primuletum carniolicae typicum, this article, Table 5.

PcPcl-CT Phyteumato columnae-Primuletum carniolicae potentilletosum caulescentis, this article, Table 7 (CT - Cerkno and Trebuša regions).

PcPcl-TG Phyteumato columnae-Primuletum carniolicae potentilletosum caulescentis (Primulo carniolicae-Potentilletum caulescentis), Dakskobler (1998, Table 1, pp. 286-287) - TG (Trnovski Gozd).

Pcty Primuletum carniolicae, Accetto (2008, Table 1, pp. 42-44).

Pccb Primuletum carniolicae caricetosum brachystachyos, Accetto (2015, Table 27, pp. 98-99).

PcPcl-As Paederoto luteae-Potentilletum caulescentis (Primulo carniolicae-Potentilletum caulescentis var. Asplenium seelosii), Accetto (2008, Table 5, pp. 49-50).

Pcvb Primuletum carniolicae violetosum biflorae, this article, Table 4.

Pc-No Primuletum carniolicae s. lat., Dolinar et al. (2017, Table 1, pp. 30-32) - No - Notranjska region

Pc-Do Primuletum carniolicae s. lat., Dolinar et al. (2013, Table 1, pp. 13-16) - Do - Dolenjska region.

Pc-Npr Primuletum carniolicae s. lat., Dolinar et al. (2015, Table 1, pp. 40-43) - Notranjski Park Cerknica.

Pc-Iv Primuletum carniolicae s. lat., Dolinar et al. (2013: Table 2, pp. 17-18) - Iv - Iški Vintgar.

PcPcl Primulo carniolicae-Potentilletum clusianae, this article, Table 8.
virostri-Pinguiculetum poldinii from the Venetian Prealps, which its authors Giovagnoli \& Tassinazzo (2012) classify into the alliance Adiantion and class Adiantetea. Given the presence of certain bryophytes, character species of this class (Hymenostylium recurvirostre, Preissia quadrata, Pellia endiviifolia and Eucladium verticillatum) in the stands of the associations Astrantio-Pinguiculetum alpinae and Astrantio-Primuletum carniolicae, these associations could be classified in the same way. Nevertheless, the fact that among the listed moss species only Hymenostylium recurvirostre has higher constancy and mean coverage, the absence of Adiantum capillus-veneris from our relevés as well as the entire species composition speak against such classification.

(Sub)montane stands of moist rock crevices with Saxifraga aizoides, which according to the hierarchical classification do not belong to the association Astrantio carniolicae-Pinguiculetum alpinae, are a curiosity in the Southeastern Alps, and we therefore classify them into the new association Campanulo cespitosae-Saxifragetum aizoidis.

The second group with the highest degree of similarity features two forms of the association Primuletum carniolicae s. lat. with the taxon Phyteuma scheuchzeri subsp. columnae and two forms of the association Primulo carniolicae-Potentilletum caulescentis. This means that similar stands from the northwestern part of Primula carniolica's distribution area are grouped together. These communities could be treated as a special geographical variant of the association Primuletum carniolicae. According to our criteria the rank of geographical variant (a small or regional association) is higher than the rank of subassociation. Since the applicable Code of Phytosociological Nomenclature (Weber et al. 2000) does not deal with this rank, we have two options for a valid typification:

- to reduce the rank of geographical variant to the rank of subassociation, or

- to bring the rank of geographical variant to the rank of association.

The results of hierarchical classification speak in favour of the latter option (see also Tables 9 and 10), as they point to certain floristic differences between the studied stands and the stands described by Accetto (2008, 2015) and Dolinar et al. $(2013,2015,2017)$ as the association Primuletum carniolicae, also in terms of the updated selection of diagnostic species (in both cases the common species are Primula carniolica, Orthothecium rufescens and Valeriana tripteris). Based on these criteria it makes sense to classify our stands into the new association Phyteumato columnae-Primuletum carniolicae. Only in this case can we take into account the results of hierarchical classification (Figure 4), which demonstrate considerable similar- 
ity of the stands of the association Primulo carniolicaePotentilletum caulescentis from the northwestern part of the distribution area of Primula carniolica (Dakskobler 1998, 2000) with presently described communities from the same area. With these findings in mind, this association should be reduced to the rank of the subassociation Phyteumato columnae-Primuletum carniolicae potentilletosum caulescentis.

Based on floristic similarity (Figure 4), however, the relevés from the rock faces of Govci under Zeleni Rob and Poldanovec, where Potentilla clusiana occurs or even dominates, cannot be classified into the association Phyteumato-Primuletum carniolicae. In the dendrogram in Figure 4 they grouped separately from other relevés and are therefore classified into the new association Primulo carniolicae-Potentilletum clusianae.

Based on the results of hierarchical classification (Figure 4, Table 9) it would make sense to rename the subassociation Primuletum carniolicae caricetosum brachystachyos (Accetto 2015) into Primuletum carniolicae caricetosum mucronatae. The nomenclatural type is the same, but Carex mucronata (frequency 100\%) defines these stands significantly more than $C$. brachystachys (frequency 50\%). However, the Code (Weber et al. 2000) does not allow such renaming, because the valid name caricetosum brachystachyos is not based on a taxonomical error (Arts. 29a and 43).

\section{Justification of the possible new suballiance (alliance) of (sub)mon- tane chasmophytic communities}

Table 9 serves also as the basis for classification of the described communities into higher syntaxonomic units. So far, these communities have been classified either into the alliance Physoplexido comosae-Saxifragion petraeae or into the alliance Cystopteridion (Šilc \& Čarni 2012, Accetto 2015, Dolinar et al. 2015, 2017). A more recent review of the vegetation of Europe at the rank of classes, orders and alliances (Mucina et al. 2016) indicates that the alliance Violo biflorae-Cystopteridion alpinae (syntax. syn. Cystopteridion) belongs to the order Violo bifloraeCystopteridetalia alpinae and class Polypodietea. This classification is different from that of Theurillat (2004), and E. and S. Pignatti $(2014,2016)$, who classify the alliance Cystopteridions into the order Potentilletalia caulescentis and class Asplenietea trichomanis. The studied communities with their entire species composition also undoubtedly belong to this order and class, and are characterised by higher occurrence of hygrophytic species. According to Mucina et al. (2016) they cannot be classified into the alliance Violo biflorae-Cystopteridion (syn. Cystopteridion). According to Theurillat (in litt.) the classification of the alliance Cystopteridion into the order Violo biflorae-Cystopteridetalia alpinae is problematic. In the original diagnosis this order corresponds to communities that are intermediate between the classes Asplenietea trichomanis and Thlaspietea rotundifoliae, and indifferent to the substrate. Therefore, the alliance Cystopteridion Richard 1972 is not the same syntaxon as the alliance Violo biflorae-Cystopteridion alpinae Fernández Casas 1970, as described in Mucina \& al. (2016).

With this in mind, we provisionally classify most of the communities described in this article into the alliance Cystopteridion and order Potentilletalia caulescentis. We also propose another possible alliance, an alliance of hygrophytic rock crevice (chasmophytic) communities of the Southeastern Alps and northern Dinaric Alps (Astrantio carniolicae-Paederotion luteae nom. prov.) an ecological vicariant of the alliance Physoplexido comosae-Saxifragion petraeae. Based on Table 9, Paederota lutea and Astrantia carniolica seem to be appropriate namegiving species for such an alliance. Paederota lutea is an eastern-Alpine-Illyrian species and a character species of chasmophytic communities (Aeschimann et al. 2004). In Slovenia it is distributed in chasmophytic communities, on screes, stony subalpine-alpine grasslands and stony shrub communities and forests from the colline to the alpine belt. In the colline, submontane and montane belts it frequently occurs on shady moist rocks and is one of the most constant companions of the endemic Primula carniolica. Surina (2005) described its association Ranunculo traunfellneri-Paederotetum luteae in the Krn Mts. and classified it into the alliance Cystopteridion. Paederota lutea is also the name-giving species of the association Paederoto luteae-Minuartietum rupestris (Wraber 1986, Surina 2005). Both described associations occur in the subalpine or alpine belt. A detailed ecological, phytogeographical and phytosociological description of Astrantia carniolica was published recently (Dakskobler \& Martinčič 2018), and is therefore not repeated herein. Although it occurs also in different communities, in dolomite headwaters, on moist screes and in stony forests, Astrantia carniolica nevertheless meets the criteria (together with Paederota lutea, which has a very similar, but slightly larger distribution area) for naming southeastern-Alpine-northern-Dinaric communities of moist rock crevices. However, to ensure that the description of the new alliance is valid, we should take into account also similar syntaxa from other parts of the Southeastern Alps and northern Dinaric Alps, and perform more extensive numerical comparisons (opinion of two anonymous reviewers, with which the authors agree). 


\section{An overview of newly described syntaxa with their nomenclatural types and diagnostic species}

Asplenietea trichomanis (Br.-Bl. in Meier et Br.-Bl. 1934) Oberd. 1977

Potentilletalia caulescentis Br.-Bl. in Br.-Bl. et Jenny 1926 Cystopteridion fragilis Richard 1972 (non. Violo biflorae-Cystopteridion alpinae sensu Mucina et al. 2016) (Astrantio carniolicae-Paederotion luteae nom. prov.)

In the context of this article the diagnostic species of this alliance include the species expected to be diagnostic also for the suggested possible new alliance: Astrantia carniolica, Paederota lutea, Primula carniolica, Orthothecium rufescens, Aster bellidiastrum, Pingiucula alpina, Carex brachystachys, Asplenium viride, Valeriana saxatilis, V. tripteris, Viola biflora, Saxifraga aizoides, Tofieldia calyculata, Cystopteris fragilis, Hymenostylium recurvirostre, Palustriella commutata.

Astrantio carniolicae-Primuletum carniolicae ass. nov. hoc loco

Nomenclatural type, holotypus, is relevé 35 in Table 1.

Diagnostic species: Primula carniolica, Astrantia carniolica, Pinguicula alpina, Hymenostylium recurvirostre, Palustriella commutata (character species) and Phyteuma scheuchzeri subsp. columnae, Molinia arundinacea, Valeriana saxatilis and Carex mucronata (differential species).

- asteretosum bellidiastri subass. nov. hoc loco (relevés 4-26 in Table 1), nomenclatural type, holotypus, is relevé 15 in Table 1.

Differential species: Aster bellidiastrum, Paederota lutea.

- hymenostylietosum recurvirostris subass. nov. hoc loco (relevés 27-56 in Table 1), nomenclatural type, holotypus, is relevé 35 in Table 1.

Differential species: Hymenostylium recurvirostre, Pinguicula alpina (because of higher medium cover value than in the stands of the former subassociation).

Relevés $1-3$ in Table 1 are classified only at the rank of the association.

Astrantio carniolicae-Pinguiculetum alpinae ass. nov. hoc loco

Nomenclatural type, holotypus, is relevé 11 in Table 2.
Diagnostic species: Pinguicula alpina, Astrantia carniolica, Hymenostylium recurvirostre, Palustriella comutata, Petasites paradoxus. var. Molinia arundinacea (relevés 13-19 in Table 2)

Campanulo cespitosae-Saxifragetum aizoidis ass. nov. hoc loco

Nomenclatural type, holotypus, is relevé 6 in Table 3.

Diagnostic species: Saxifraga aizoides, Campanula cespitosa, Molinia arundinacea, Palustriella commutata, Calamagrostis varia, Preissia quadrata.

Variants:

var. Pinguicula alpina (relevés 1-2 in Table 3): differential species Pinguicula alpina, Carex flacca

var. Caltha palustris (relevés 3-4 in Table 3): differential species Caltha palustris, Gymnostomum aeruginosum.

var. Hymenostylium recurvirostre (relevés 5-9 in Table 3): differential species Hymenostylium recurvirostre, Tofieldia calyculata. var. Aster bellidiastrum (relevés 10-14 in Table 3): differential species Aster bellidiastrum, Orthothecium rufescens, Veronica urticifolia, Saxifraga cuneifolia.

Phyteumato columnae-Primuletum carniolicae ass. nov. hoc loco

Nomenclatural type, holotypus, is relevé 28 in Table 5.

Diagnostic species: Primula carniolica, Phyteuma scheuchzeri subsp. columnae, Paederota lutea, Orthothecium rufescens, Valeriana tripteris, Carex bracyhstachys.

- typicum subass. nov. hoc loco (nomenclatural type is the same as the nomenclatural type of the association, relevé 28 in Table 5)

var. Viola biflora (relevés 1-3 in Table 5), differential species Viola biflora, Cystopteris fragilis.

- astrantietosum carniolicae subass. nov. hoc loco

Nomenclatural type, holotypus, is relevé 17 in Table 6.

Differential species: Astrantia carniolica, Viola biflora, Valeriana saxatilis.

- potentilletosum caulescentis (Dakskobler (1998) 2000) subass. nov. stat. nov.

(basionym Primulo carniolicae-Potentilletum caulescentis Dakskobler (1998) 2000, 
see Dakskobler 1998: 272-273, 286-287; 2000: 56)

Nomenclatural type, lectotypus, is relevé 1 in Table 1 (Dakskobler 1998: 286-287).

Differential species: Potentilla caulescens, Erica carnea, Polygala chamaebuxus, Primula auricula, $P . \times$ venusta.

Primulo carniolicae-Potentilletum clusianae ass. nov. hoc loco

Nomenclatural type, holotypus, is relevé 4 in Table 8.

Diagnostic species: Potentilla clusiana, Primula carniolica, Carex firma, Campanula cochleariifolia, Rhodothamnus chamaecistus, Hladnikia pastinacifolia, Hieracium pillosum, Aquilegia iulia.

Primuletum carniolicae Accetto 2008 violetosum biflorae subass. nov. hoc loco

Nomenclatural type, holotypus, is relevé 11 in Table 4.

Differential species: Viola biflora, Pinguicula alpina, Veronica urticifolia.

Physoplexido comosae-Saxifragion petraeae Mucina et Theurillat 2015

Paederoto luteae-Potentilletum caulescentis Accetto ex Dakskobler et Martinčič ass. nov. hoc loco

(basionym Primulo carniolicae-Potentilletum caulescentis Dakskobler (1998) 2000 var. Asplenium seelosii Accetto 2008)

Nomenclatural type, lectotypus, is relevé 6 in Table 5 (Accetto 2008: 49-50).

Diagnostic species: Potentilla caulescens, Paederota lutea, Primula carniolica, Asplenium seelosii.

Note: Based on the synoptic table (Table 9) and Figure 4 we reduced the rank of the association Primulo carniolicae-Potentilletum caulescentis Dakskobler (1998) 2000 to the rank of the subassociation Phyteumato-Primuletum carniolicae potentilletosum caulescentis. As this subassociation clearly does not comprise the syntaxon Primulo carniolicae-Potentilletum caulescentis var. Asplenium seelosii Accetto 2008 (see Figure 4), we gave it a new name and described a new association, whose author is M. Accetto.

The stands of the association Primuletum carniolicae s. lat., which were studied in the central and partly in the southwestern part of the distribution area of Primula carniolica by Dolinar et al. (2013, 2015, 2017), differ from the stands of the association Primuletum carniolicae described by Accetto $(2008,2015)$ mainly because they comprise Aruncus dioicus, Cystopteris fragilis, Moehringia muscosa, Pinguicula alpina, Hieracium bifidum, Polystichum aculeatum and Scopolia carniolica, which indicates slightly moister sites, and in percentages of the groups of diagnostic species (a higher percentage of the species of the class Vaccinio-Piceetea, order Fagetalia sylvaticae and alliances Aremonio-Fagion and Tilio-Acerion - Table 10). For the time being, however, we do not describe them at the level of subassociation.

\section{Ecology of the studied communities}

\section{Astrantio carniolicae-Primuletum carniolicae}

Distribution area of the stands of this association is shown in Figure 5. They are located in Orehovska Grapa gorge (under Orehek), in Kazarska Grapa (in particular in Beli Potok) under Bukovo and Bukovski Vrh, and in Poličnica at Police (all of them in the Cerkno region, in the pre-Alpine phytogeographical region), and were recorded also in the Trebuša valley, along the Trebušica, Gačnik, Srna Grapa and Makčeva Grapa gorges, in gorges under Bukov Vrh, Poldanovec and Stanov Rob (Govci), and in Govškarca in the Kanomlja valley. The localities occur at elevations ranging from 230 to $760 \mathrm{~m}$, with a little more than a half of the relevés on shady, and the other on sunny aspects. The parent material is mainly dolomite, with occasional tufa formations, and the average number of species per relevé is 16 (in the range of 7 to 29 species).

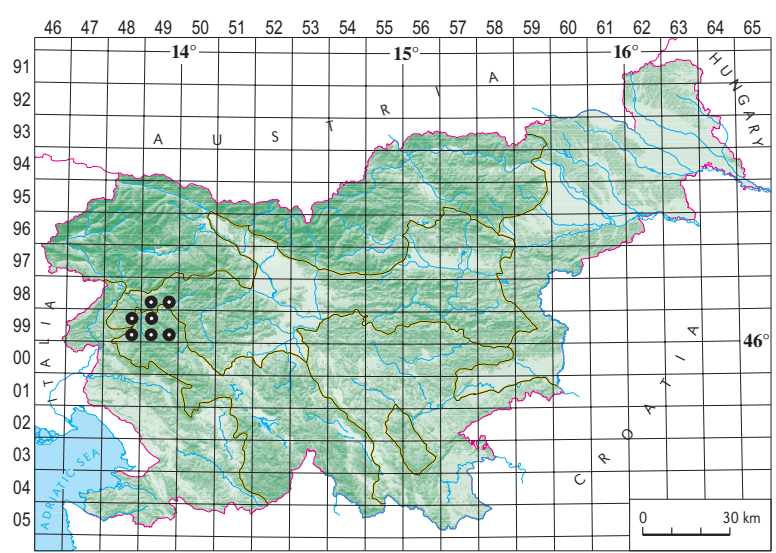

Figure 5: Distribution area of the stands of the association Astrantio carniolicae-Primuletum carniolicae on the map of Slovenia.

Slika 5: Območje razširjenosti sestojev asociacije Astrantio carniolicae-Primuletum carniolicae na zemljevidu Slovenije. 


\section{Astrantio carniolicae-Pinguiculetum alpinae}

The distribution area of the stands of this association is shown in Figure 6. They are located in Tolmin Gorge, in the Soča ravine under Magozd (at Otona pool and Globoščak gorge), in Velike Luti (Kneža valley), in the Bača ravine at Klonte and Humar (between Koritnica and Hudajužna), at Brinta waterfall at Selce, in Dabrček gorge under the Šentviška Gora Plateau, in Orehovska Grapa, in the Hotenja valley, the Trebuša valley (at the Trebušica and Gačnik streams). The stands of the association were recorded at elevations ranging from 205 to $590 \mathrm{~m}$, with half of the relevés on shady, and the other half on sunny aspects. The parent material is dolomite, limestone, breccia, in places admixture of marlstone and chert, and in some spots tufa formations. The average number of species per relevé is 12 (in the range of 6 to 30 species).

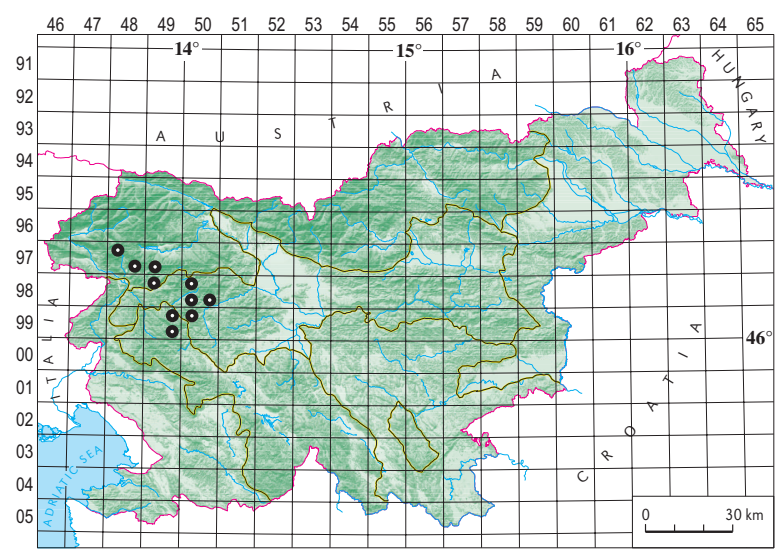

Figure 6: Distribution area of the stands of the association Astrantio carniolicae-Pinguiculetum alpinae on the map of Slovenia.

Slika 6: Območje razširjenosti sestojev asociacije Astrantio carniolicae-Pinguiculetum alpinae na zemljevidu Slovenije.

\section{Campanulo cespitosae-Saxifragetum aizoidis}

The distribution area of the stands of this association is shown in Figure 7. They are located in the upper course of the Volarja river under Gregorčič waterfall, at the stream Godiča at Beri waterfall and downstream toward Poljubinj, in Prodarjeva Grapa gorge under the village of Porezen, in gorges Linderpoh (under Šprickovbl waterfall) and Kacenpoh at Bača near Podbrdo, in the Zadlaščica river valley at Perbla and in the Tolminka valley (at the Zala gorge). It was recorded at elevations ranging from 255 to $990 \mathrm{~m}$, most often on southeastern and northeastern aspects. The predominant geological bedrock is limestone with an admixture of marlstone and chert, occasionally also dolomite with chert. The average number of species per relevé is 17 (in the range of 10 to 26 spe- cies). The Arctic-Alpine species Saxifraga aizoides is characteristic primarily for moist stony sites in the subalpine and alpine belt; it is distributed only in the Alpine phytogeographical region, with several localities also in the pre-Alpine phytogeographical region: Črni vrh above the stream of Batava, Porezen, Prodarjeva Grapa gorge and the Bača valley between Humar and Klonte (Figure 7). Its localities in the valleys of the Godiča, Volarja and Bača and along the Soča under Magozd are among the lowestlying in Slovenia (comp. Dakskobler et al. 2017b). In Slovenia, the eastern-Alpine species Campanula cespitosa is distributed also beyond the Alps, in the pre-Alpine and Dinaric phytogeographical regions (Figure 8) and is not rare on rocky and gravelly areas in the submontane belt.

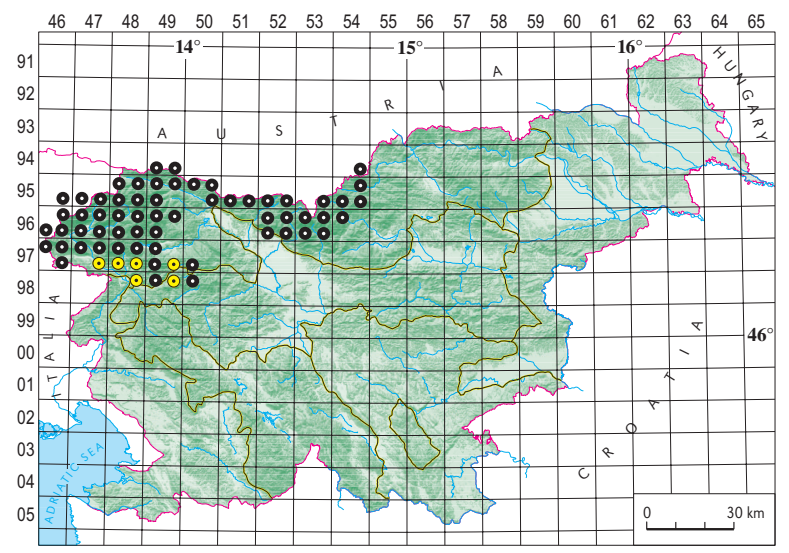

Figure 7: Distribution of Saxifraga aizoides $\bullet$ (FloVegSi database) and of the stands of the association Campanulo cespitosae-Saxifragetum aizoidis $\odot$ in Slovenia.

Slika 7: Razširjenost vrste Saxifraga aizoides $\bullet$ (podatkovna baza FloVegSi) in sestojev asociacije Campanulo cespitosae-Saxifragetum aizoidis $\odot$ na zemljevidu Slovenije.

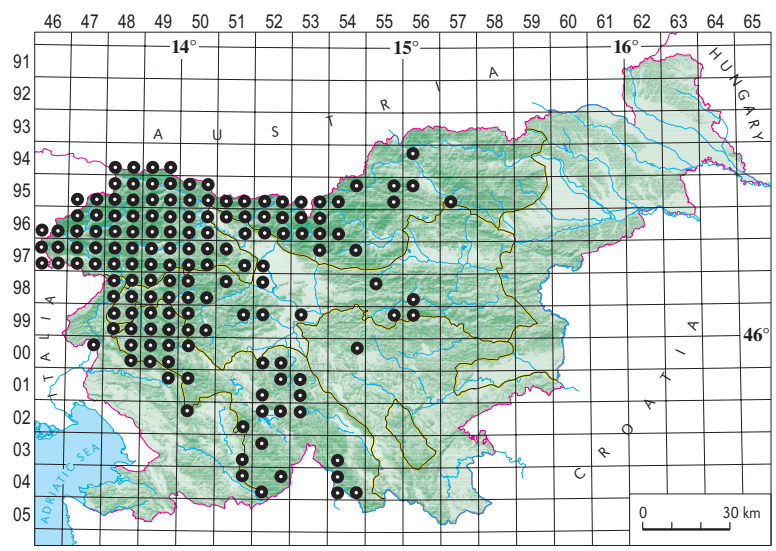

Figure 8: Distribution of Campanula cespitosa in Slovenia (FloVegSi database).

Slika 8: Razširjenost vrste Campanula cespitosa v Sloveniji (podatkovna baza FloVegSi). 


\section{Phyteumato columnae-Primuletum carniolicae typicum}

The distribution area of the stands of this subassociation is shown in Figure 9. They are located in the rock faces of Maslenica above the saddle of Oblakov Vrh, in the Trebuša valley (Gačnik, Pršjak, Srna Grapa), under Skopica above the Idrijca valley, under Vrše, Drnulk and Kobilica above the Trebuša valley, under Drnulk and the Stador-Kobilica ridge above the Čepovan valley, in the Sjavnica (Sevnica) gorge under the village of Jagršče, under the hill of Degarnik above Beli Potok gorge and in gorges of Kazarska Grapa and Orehovska Grapa, in Govškarca and V Studencu above the Kanomlja valley, under Sončni Rob above Hudo Polje at Vojsko, under Mali Golak. It was recorded at elevations ranging from 215 to $1460 \mathrm{~m}$ (Mali Golak) on predominantly shady aspects. This explains the large elevation range of the localities - in the Trebuša valley at around $200 \mathrm{~m}$ a.s.l., for example, Primula carniolica can be accompanied also by the subalpine species $R h o-$ dodendron hirsutum and Rhodothamnus chamaecistus, and on similar localities and sites Carex ferruginea also occurs at very low elevations. Site conditions in moist and shady gorges of the Trebušica, Gačnik and some other streams with a cold local climate are similar to those in the altimontane belt. The parent material is almost exclusively dolomite. The average number of species per relevé is 17 (in the range of 6 to 34 species).

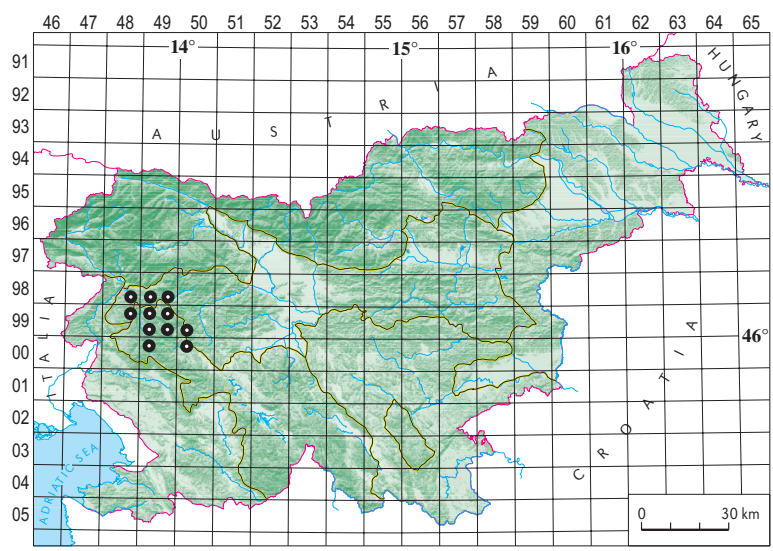

Figure 9: Distribution area of the stands of the subassociation Phyteumato columnae-Primuletum carniolicae typicum on the map of Slovenia. Slika 9: Območje razširjenosti sestojev subasociacije Phyteumato columnae-Primuletum carniolicae typicum na zemljevidu Slovenije.

\section{Phyteumato columnae-Primuletum carniolicae astrantietosum carniolicae}

The distribution area of the stands of this subassociation is shown in Figure 10: on the Trnovski Gozd Plateau: Sončni Rob above Hudo Polje, under Mts. Mali and Srednji Golak, under Bukov Vrh and Mali Govci; in
Gačnik and Makčeva Grapa gorges in the Trebuša valley, under the hill of Hudournik above the Hotenja valley. It was recorded at elevations ranging from 215 to $1440 \mathrm{~m}$, with most of the relevés at $750 \mathrm{~m}$ a.s.l., predominantly on shady aspects. The parent material is dolomite, rarely dolomite limestone. The average number of species per relevé is 18 (in the range of 11 to 24 species).

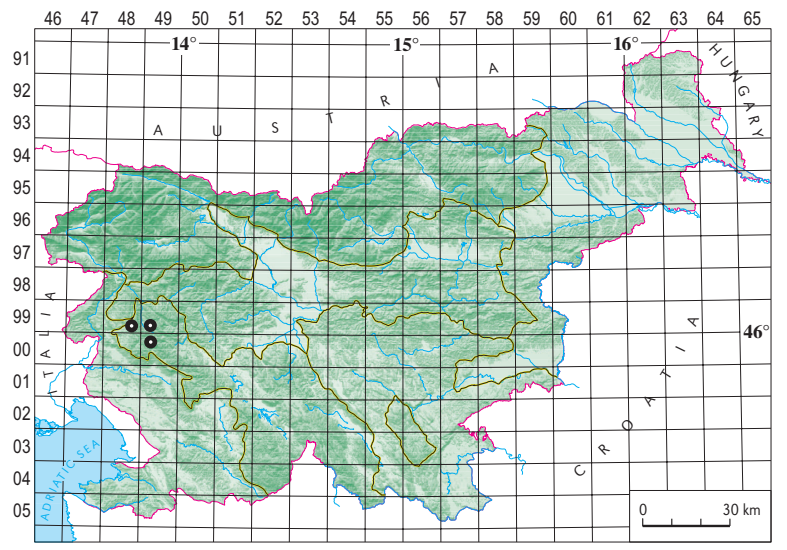

Figure 10: Distribution area of the stands of the subassociation Phyteumato columnae-Primuletum carniolicae astrantietosum carniolicae on the map of Slovenia.

Slika 10: Območje razširjenosti sestojev subasociacije Phyteumato columnae-Primuletum carniolicae astrantietosum carniolicae na zemljevidu Slovenije.

\section{Phyteumato columnae-Primuletum carniolicae potentilletosum caulescentis}

The distribution area of the stands of this subassociation is shown in Figure 11: in Orehovska Grapa gorge, in the Idrijca valley under Dolenji Potoki (Straža) and under Sv. Ivan (Šebrelje), under Skopica, Hudournik

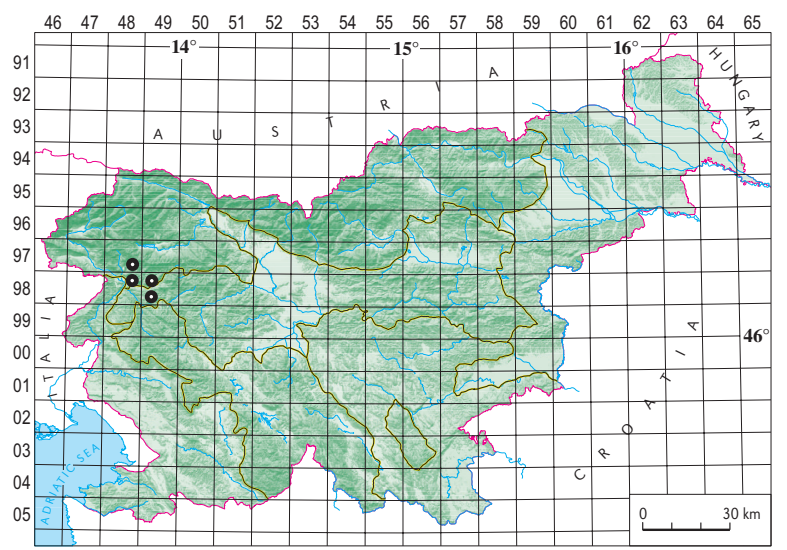

Figure 11: Distribution area of the stands of the subassociation Phyteumato columnae-Primuletum carniolicae potentilletosum caulescentis on the map of Slovenia.

Slika 11: Območje razširjenosti sestojev subasociacije Phyteumato columnae-Primuletum carniolicae potentilletosum caulescentis na zemljevidu Slovenije. 
and Poldanovec, under Drnulk and Kobilica above the Trebuša valley. Its localities are situated at elevations ranging from 240 to $1050 \mathrm{~m}$, with most of them above $550 \mathrm{~m}$ a.s.l. Aspects are shady, the parent material is dolomite. The average number of species per relevé is 19 (in the range of 12 to 31 species).

\section{Primulo carniolicae-Potentilletum clusianae}

The localities of the stands of this association are under Zeleni Rob and Poldanovec on the northern edge of the Trnovski Gozd Plateau (Figure 12). Elevations range between 1170 and $1330 \mathrm{~m}$, the parent material is dolomite, aspect shady. The average number of species per relevé is 17 (in the range of 13 to 23 species). In terms of species composition the stands of this association show consider-

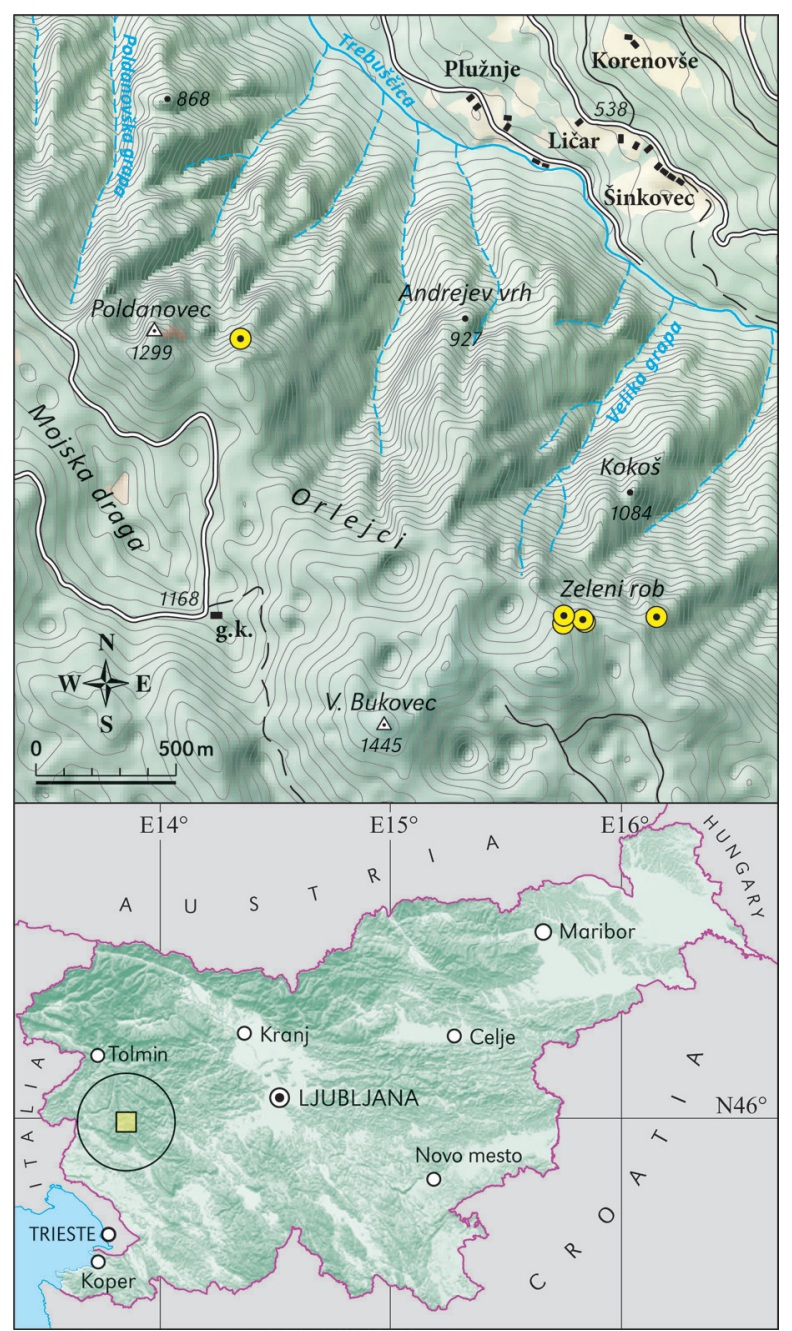

Figure 12: Localities of the stands of the association Primulo carniolicae-Potentilletum clusianae on the northern edge of the Trnovski Gozd Plateau.

Slika 12: Nahajališča sestojev asociacije Primulo carniolicae-Potentilletum clusianae na severnem robu Trnovskega gozda. able similarity and in places contact with the stands of the association Primulo carniolicae-Caricetum firmae (Dakskobler 2006), but are substantially different in terms of mean coverage and constancy of certain edifier species. In the stands of the association Primulo-Caricetum firmae the species Carex firma and Sesleria caerulea occur at similar constancy to that in the stands of the association PrimuloPotentilletum clusianae, but with a visibly higher mean coverage, and Potentilla clusiana occurs with a substantially lower constancy. There are obvious differences also in the constancy and mean coverage of Aster bellidiastrum, Campanula cespitosa (higher in the first compared association), C. cochleariifolia, Hladnikia pastinacifolia, Hieracium pilosum (higher in the second compared association), in the average number of species per relevé (higher in the first compared association) and in mean coverage (cover) of the herb layer (visibly higher in the first compared association) - comp. Dakskobler (2006, Table 1, relevés 1-23, pp. 90-99). Into the association Primulo carniolicae-Potentilletum clusianae we classify stands of moist rock crevices (as demonstrated also by percentages of groups of diagnostic species, Table 10), whereas the stands of stony subalpine grasslands are classified into the association Primulo carniolicae-Caricetum firmae.

\section{Primuletum carniolicae violetosum biflorae}

The distribution area of the stands of this subassociation is shown in Figure 13. Most of the relevés were made in the Raskovec gorge in the Žirovnica valley at Žiri (in Idrija municipality), several relevés also in the gorge of Gačnik and the Govškarca above the Kanomlja valley. It was recorded at elevations ranging from 430 to $850 \mathrm{~m}$ on predominantly shady aspects. The parent material is dolomite. The average number of species per relevé is 20 (in the range of 7 to 28 species).

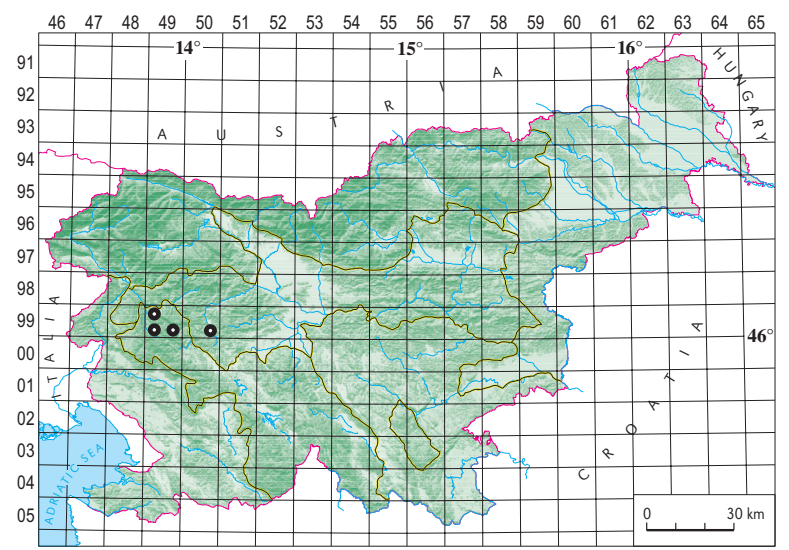

Figure 13: Distribution area of the stands of the subassociation Primuletum carniolicae violetosum biflorae on the map of Slovenia. Slika 13: Območje razširjenosti sestojev subasociacije Primuletum carniolicae violetosum biflorae na zemljevidu Slovenije. 


\section{Nature conservation status}

Primula carniolica is listed in Appendix 2 of the Council Directive 92/43/EEC on the Conservation of Natural Habitats and of Wild Fauna and Flora - 1992 (Dakskobler et al. 2004). In the study area, i.e. in the northwestern part of its distribution area, this species occurs the most abundantly in shady gorges in the Cerkno region (Kazarska Grapa, Orehovska Grapa, Poličnica, Sjavnica, on the northeastern edge of the Šentviška Gora Plateau under Degarnik and on the pasture at the homestead Seljak, in rock faces under Sv. Ivan near Šebrelje above the Idrijca valley) and in the Trebuša valley (Gačnik, Makčeva Grapa, Srna Grapa, Pršjak, Mali Govci, also along the Trebušica and the gorges that flow into this river on the left bank), and in the rock faces of Govci from Bukov Vrh across Zeleni Rob, Poldanovec and Stanov Rob to Stador, Drnulk and Skopica). The population is abundant and vital, and has not yet been affected by the human impact, except on isolated localities. Also abundant is the population in the Raskovec gorge in the Železnica valley in the Žiri region (Dakskobler et al. 2017a, Dolinar et al. 2017), in Govškarca, Klavžarica and V Studencu above the Kanomlja valley. Another two species of European conservation concern sporadically occur in the studied communities of moist rock crevices, namely Aquilegia iulia and Hladnikia pastinacifolia (Čušin et al. 2004, Podobnik et al. 2013) as well as a number of protected species (Anon. 2004): Cyclamen purpurascens, Gentiana clusii, Gymnadenia odoratissima, Hemerocallis lilioasphodelus, Leontopodium alpinum, Pinguicula alpina, Primula auricula, Primula $\times$ venusta, and Taxus baccata as well as several other Red List species (Anon. 2002): Schoenus nigricans, Veratrum nigrum. Unlike flowering plants, bryophytes do not comprise rare or phytogeographically important species, nor do they comprise species from the Slovenian (Martinčič 2016) or European Red List (Hodgetts et al. 2019). The studied communities belong to Natura 2000 type 8210 Calcareous rocky slopes with chasmophytic vegetation, and the stands of associations Astrantio-Primuletum and Astrantio-Pinguiculetum also to Natura 2000 habitat type 7220 Petrifying springs with tufa formation (Cratoneurion) - Jogan et al. (2004).

\section{Conclusions}

A large number of the relevés made in the moist rocks in the submontane and montane belt (mainly in the area of Illyrian beech forests from the alliance Aremonio-Fagion) in western Slovenia between the southern Julian Alps and the northern Dinaric Alps can be arranged in tables in different ways. Our classification followed certain field findings where it was established that as a rule only a few dominant species occur with high mean coverage in these special site conditions. In the predominantly dolomite bedrock it is the moisture conditions that determine which species will dominate. In the southern Julian Alps, where the bedrock comes also as platy limestones with an admixture of marlstone and chert, this depends on other factors as well. With this in mind, it makes sense to classify the stands with Primula carniolica on the moistest rocks into the association Astrantio carniolicae-Primuletum carniolicae, because Pinguicula alpina does not have as high constancy and mean coverage here as in the stands of a similar association, Astrantio-Pinguiculetum alpinae, in which Primula carniolica does not occur, as these stands are, at least in part, outside its distribution area.

All other relevés of rock crevices with Primula carniolica could be classified into the association Primuletum carniolicae (in the broadest sense), which was first recorded in Slovenia and named by T. Wraber (he found it at Divje Jezero / Wild Lake at Idrija already on 16/8/1973, and later in the ravine of Kadice at Sodražica on 25/4/1982). Unfortunately, Wraber never processed or published his relevés and we only came across them by chance in 2016 and 2017 when we studied his legacy, which is kept at Wraber's Library at the University of Ljubljana's Botanical Garden. Tone Wraber was without a doubt a pioneer in the phytosociological investigation of chasmophytic communities with Primula carniolica, but his relevés cannot have been known to Accetto (2008), who was the first to validly publish the description of the association Primuletum carniolicae. The relevés of the association Primuletum carniolicae in the northwestern part of the distribution area are nevertheless slightly different from the relevés of this association in the central and southwestern part of its distribution area. The most appropriate syntaxonomic solution would be to describe a new geographical variant with the subspecies Phyteuma scheuchzeri subsp. columnae. However, given the results of hierarchical classification we could also describe a new association, Phyteumato-Primuletum carniolicae, which would allow us to evaluate the established variability of individual stands at the rank of subassociations and not only at the rank of variants. Wraber's notebook comprises a relevé dated 3 May 1982, locality Strug, rocks on the right side of the road from Fežnar toward Divje Jezero lake, which comprises most of the diagnostic species of the subassociation Phyteumato-Primuletum carniolicae astrantietosum carniolicae. The decision for an independent association Phyteumato-Primuletum carniolicae, on the other hand, means reducing the rank of the association Primulo carniolicae-Potentilletum caulescentis to the rank 
of subassociation (which could not happen if we insisted on the association Primuletum carniolicae in the broadest sense - comp. Accetto 2008). Because Primula carniolica characterises very hygrophytic chasmophytic communities it is rightly considered a character species of the alliance Cystopteridion (not Violo biflorae-Cystopteridion sensu Mucina et al. 2016), into which we provisionally classify most of the described new syntaxa (except for association Paederoto luteae-Potentilletum caulescentis, which is classified into the alliance Physoplexido comosae-Saxifragion petraeae). The article also proposes a new possible alliance of hygrophytic rock crevice (chasmophytic) communities of the Southeastern Alps and northern Dinaric Alps, an ecological vicariant of the alliance Physoplexido comosae-Saxifragion petraeae. The name-giving species for such an alliance could be Paederota lutea and Astrantia carniolica (Astrantio carniolicae-Paederotion luteae nom. prov.). However, a valid description of a new alliance requires further research that takes into account also similar syntaxa of hygrophytic chasmophytic communities from other parts of the Southeastern Alps and northern Dinaric Alps, and more extensive numerical comparisons.

\section{Povzetek}

\section{Združbe vlažnih skalnih razpok z endemitom Primula carniolica v (sub)montanskem pasu zahodne Slovenije}

Primula carniolica, evropsko varstveno pomembna vrsta (Natura 2000), je v severozahodnem delu svojega areala (v zahodni Sloveniji med južnimi Julijskimi Alpami in severnim delom Dinarskega gorstva) najbolj množično prisotna $\mathrm{v}$ senčnih grapah na Cerkljanskem (Kazarska grapa, Orehovska grapa, Poličnica, Sjavnica, v ostenjih pod sv. Ivanom nad dolino Idrijce pod Šebreljami, tudi na severovzhodnem robu Šentviške planote: pod Degarnikom in na pašniku pri domačiji Seljak v Bukovskem Vrhu, že na Tolminskem) in v dolini Trebuše (Gačnik, Makčeva grapa, Srna grapa, Pršjak, Mali Govci, tudi ob sami Trebušici in grapah, ki se vanjo izlivajo na levem bregu) ter v ostenju Govcev od Bukovega vrha preko Zelenega roba, Poldanovca in Stanovega roba vse do Stadorja, Drnulka in Skopice). Populacija je bogata, vitalna in za zdaj človek nanjo razen na nekaterih nahajališčih nima večjega vpliva. Bogata je tudi populacija $v$ grapi Raskovec v dolini Železnice na Žirovskem in v Govškarci, Klavžarici in V Studencu nad dolino Kanomlje.

Veliko število fitocenoloških popisov, ki smo jih naredili v vlažnem skalovju v submontanskem in montanskem pasu (večinoma v območju ilirskih bukovih gozdov iz zveze Aremonio-Fagion) v raziskovanem območju je mogoče $v$ tabele razvrščati na več načinov. Naša ureditev je sledila nekaterim spoznanjem na terenu, kjer je očitno, da je v teh posebnih rastiščnih razmerah navadno le malo prevladujočih vrst $\mathrm{z}$ večjim srednjim zastiranjem. Katere so, pa je v prevladujoči dolomitni podlagi predvsem odvisno od vlažnostnih razmer. $\mathrm{V}$ južnih Julijskih Alpah, kjer so podlaga tudi ploščasti apnenci s primesjo laporovca in roženca, lahko nanjo vplivajo tudi drugi dejavniki. Ob tej podmeni je uvrstitev sestojev z vrsto Primula carniolica na najbolj vlažnem skalovju v asociacijo Astrantio carniolicae-Primuletum carniolicae smiselna, saj v njej vrsta Pinguicula alpina nima tako velike stalnosti in srednjega zastiranja kot $\mathrm{v}$ sestojih podobne asociacije Astrantio-Pinguiculetum alpinae, v kateri pa vrsta Primula carniolica ne uspeva in so ti sestoji vsaj deloma tudi zunaj njenega areala.

Vse ostale popise skalnih razpok, kjer uspeva vrsta Primula carniolica, bi lahko uvrstili v široko zajeto asociacijo Primuletum carniolicae, ki jo je v Sloveniji prvi popisal in tako imenoval T. Wraber (pri Divjem jezeru pri Idriji že 16. 8. 1973, v soteski Kadice pri Sodražici 25. 4. 1982). Žal popisov ni nikoli obdelal ali objavil in smo nekatere od njih slučajno opazili v letih 2016 in 2017 v njegovi zapuščini, ki jo hrani Wraberjeva knjižnica v Botaničnem vrtu Univerze v Ljubljani. Vsekakor je bil Tone Wraber pionir pri fitocenološkem raziskovanju združb skalnih razpok z vrsto Primula carniolica, a njegovih popisov Accetto (2008), ki je prvi veljavno objavil opis asociacije Primuletum carniolicae ni mogel poznati. Popisi asociacije Primuletum carniolicae v severozahodnem delu areala se vendarle nekoliko razlikujejo od popisov te asociacije v osrednjem in jugozahodnem delu areala. Najprimernejša sintaksonomska rešitev bi bil opis nove geografske variante s podvrsto Phyteuma scheuchzeri subsp. columnae. Toda rezultati hierarhične klasifikacije dopuščajo tudi opis nove asociacije Phyteumato-Primuletum carniolicae, ki omogoča, da tudi ugotovljeno variabilnost posameznih sestojev vrednotimo na rangu subasociacij in ne zgolj na rangu variant. $\mathrm{V}$ Wraberjevi beležnici, datum popisa je 3. 5. 1982, nahajališče pa Strug, skalovje na desni strani ceste od Fežnarja proti Divjemu jezeru, je popis, ki vsebuje večino diagnostičnih vrst subasociacije Phyteumato-Primuletum carniolicae astrantietosum carniolicae. Odločitev za samostojno asociacijo Phyteumato-Primuletum carniolicae pa ima za posledico tudi znižanje ranga asociacije Primulo carniolicae-Potentilletum caulescentis na rang subasociacije (kar bi, če bi ostali pri široko zajeti asociaciji Primuletum carniolicae, ne mogli storiti - prim. Accetto 2008). Ker vrsta Primula carniolica označuje precej vlagoljubne združbe skalnih razpok, jo upravičeno štejemo za značilnico zveze Cystopteridion (ne Violo biflorae-Cystopteridion v smislu Mucina et al. 2016), v katero 
za zdaj uvrščamo večino novo opisanih sintaksonov (razen asociacije Paederoto luteae-Potentilletum caulescentis, ki jo uvrščamo v zvezo Physoplexido comosae-Saxifragion petraeae). V članku razmišljamo tudi o novi zvezi združb vlažnih skalnih razpok v Jugovzhodnih Alpah in severnem delu Dinarskega gorstva, ki bi bila ekološka vikarianta zvezi Physoplexido comosae-Saxifragion petraeae. Primerni vrsti za poimenovanje take zveze sta vrsti Paederota lutea in Astrantia carniolica (Astrantio carniolicae-Paederotion luteae nom. prov.). Za veljaven opis takšne zveze bi bile potrebne nadaljnje raziskave, predvsem pa upoštevanje podobnih sintaksonov iz drugih delov Jugovzhodnih Alp in Dinarskega gorstva in bolj obsežna primerjava $\mathrm{z}$ numeričnimi metodami.

$\mathrm{V}$ preučenih združbah vlažnih skalnih razpok ponekod uspevata še dve evropsko varstveni pomembni vrsti Aquilegia iulia in Hladnikia pastinacifolia in večje število zavarovanih vrst: Cyclamen purpurascens, Gentiana clusii, Gymnadenia odoratissima, Hemerocallis lilioasphodelus, Leontopodium alpinum, Pinguicula alpina, Primula auricula, Primula $\times$ venusta in Taxus baccata in nekaterih drugih vrst iz rdečega seznama: Schoenus nigricans, Veratrum nigrum. Preučene združbe sodijo v Natura 2000 habitatni tip 8210 Karbonatna skalnata pobočja z vegetacijo skalnih razpok, sestoji asociacij Astrantio-Primuletum in Astrantio-Pinguiculetum tudi v Natura 2000 habitatni tip 7220 Lehnjakotvorni izviri (Cratoneurion).

\section{Acknowledgements}

The authors owe special thanks to Dr. Branko Vreš, Mag. Andrej Seliškar, Brane Anderle, Janez Mihael Kocjan and Branko Dolinar, co-authors of Figures 1, 3, 8 and 9. Iztok Sajko prepared Figures 1-13 and Prof. Dr. Andrej Rozman Figure 14 (Appendix) for print. We would like to thank the heirs of late Tone Wraber for giving his manuscripts and professional literature to the safekeeping of the Botanical Garden of the University of Ljubljana, and to its director, Dr. Jože Bavcon, who allowed us to examine professor's legacy. Sincere thanks to Prof. Dr. Jean-Paul Theurillat for his valuable data and advice about the ranking of new syntaxa. Three anonymous reviewers helped us with valuable improvements and corrections. We also acknowledge the financial support from the Slovenian Research Agency (research core funding No. P1-0236). English translation by Andreja Šalamon Verbič.

\section{References}

Accetto, M. 2008: Floristične in vegetacijske zanimivosti z ostenij na severnih, severozahodnih in zahodnih pobočjih doline potoka Prušnice (0152/1, del). Razprave 4. razreda SAZU 49-1: 5-53.

Accetto, M. 2010: Rastlinstvo Iškega Vintgarja. Praprotnice in semenke. Folia biologica et geologica 51 (4): 5-149.

Accetto, M. 2013: Rastlinstvo in deloma rastje soteske Zale v zgornjem porečju Iške. Zbornik gozdarstva in lesarstva 99: 3-149.

Accetto M. 2015: Gozdno in drugo rastje na levem bregu Iškega vintgarja. Forest and other plant communities on the Iški vintgar left bank. Acta silvae et ligni 106: 1-121.

Aeschimann, D., Lauber, K., Moser, D. M. \& Theurillat, J.-P. 2004: Flora alpina. Bd. 1, 2, 3. Haupt Verlag, Bern, Stuttgart, Wien, 1159 pp., 1188 pp, 322 pp.

Anonymous, 2002: Pravilnik o uvrstitvi ogroženih rastlinskih in živalskih vrst v rdeči seznam. Uradni list RS 82/2002.

Anonymous, 2004: Uredba o zavarovanih prosto živečih rastlinskih vrstah. Uradni list RS 46/2004.

Braun-Blanquet, J. 1964: Pflanzensoziologie. Grundzüge der Vegetationskunde. 3. Auflage. Springer, Wien - New York, 865 pp.

Buser, S. 2009: Geološka karta Slovenije 1: 250.000. Geological map of Slovenia 1.250,000. Geološki zavod Slovenije, Ljubljana.

Čušin, B. (ed.), Babij, V., Bačič, T., Dakskobler, I., Frajman, B., Jogan, N., Kaligarič, M., Praprotnik, N., Seliškar, A., Skoberne, P., Surina, B., Škornik, S. \& Vreš, B. 2004: Natura 2000 v Sloveniji, Rastline. Založba ZRC, Ljubljana, 172 pp.

Dakskobler, I. 1998: Vegetacija gozdnega rezervata Govci na severovzhodnem robu Trnovskega gozda (zahodna Slovenija). In: J. Diaci (ed..): Gorski gozd. Zbornik referatov. 19. gozdarski študijski dnevi, Logarska dolina 26.-27. 3. 1998, Ljubljana, pp. 269-301.

Dakskobler, I. 1999: Contribution to the knowledge of the association Fraxino orni-Pinetum nigrae Martin-Bosse 1967. Wissenschaftliche Mitt. Niederösterr. Landesmuseum (St. Pölten) 12: 25-52.

Dakskobler, I. 2000: Fitocenološka oznaka rastišč endemične vrste Moehringia villosa (Wulfen) Fenzl (Caryophyllaceae). Razprave 4. razreda SAZU 41-2: 41-93.

Dakskobler, I. 2003: Asociacija Rhododendro hirsuti-Fagetum Accetto ex Dakskobler 1998 v zahodni Sloveniji. Razprave 4. razreda SAZU 44-2: 5-85.

Dakskobler, I. 2006: Calcareous open sedge swards and stony grasslands (Seslerietalia caeruleae) on the northern edge of the Trnovski gozd plateau (the Dinaric mountains, western Slovenia). Hacquetia 5 (1): 73-112.

Dakskobler, I. 2007: Gozdovi plemenitih listavcev v Posočju. Forest of valuable broad-leaved tree species in the Soča Valley (western Slovenia). Scopolia 60: 1-287.

Dakskobler, I. 2008: Združbe visokih steblik v Julijskih Alpah in v severnem delu Trnovskega gozda (severozahodna in zahodna Slovenija). Tall herb communities in the Julian Alps and in the northern part of the Trnovski gozd plateau (northwestern and western Slovenia). Razprave 4. razreda SAZU 49-1: 57-164. 
Dakskobler, I. 2010: Razvoj vegetacije na prodiščih reke Idrijce v zahodni Sloveniji. Development of vegetation on gravel sites of the Idrijca River in western Slovenia, Folia biologica et geologica 51 (2): 5-90.

Dakskobler, I. 2015a: Phytosociological analysis of montane beech forests on steep shady slopes on mixed geological bedrock in western Slovenia. Folia biologica et geologica 56 (1): 8-103.

Dakskobler, I. 2015b: Phytosociological description of Ostrya carpinifolia and Fraxinus ornus communities in the Julijan Alps and in the northern part of the Dinaric Alps (NW and W Slovenia, NE Italy). Hacquetia 14 (2): 175-247.

Dakskobler, I., Urbančič, M. \& Wraber, A. 2000: Gozd bukve in jelke $\mathrm{z}$ dlakavim slečem (Omphalodo-Fagetum rhododendretosum hirsuti) v Trnovskem gozdu (zahodna Slovenija). Zbornik gozdarstva in lesarstva 62: 5-52.

Dakskobler, I., Frajman, B. \& Jogan, N. 2004: Primula carniolica L. - kranjski jeglič. In: Čušin, B. (ed.) \& al.: Natura 2000 v Sloveniji rastline, ZRC, ZRC SAZU, Ljubljana, pp. 135-140.

Dakskobler, I \& Vreš, B. 2014: Ekološke značilnosti, razširjenost in ohranitvena stanja evropsko varstveno pomembnih praprotnic in semenk, ki uspevajo v gozdovih Slovenije. Gozdarski vestnik 72 (10): $440-451$.

Dakskobler, I. \& Surina, A. 2017a: Phytosociological analysis of alpine swards and heathlands (pioneer patches) on ridges and peaks in the Julian Alps (NW Slovenia). Hacquetia 16 (1): 49-171.

Dakskobler, I. \& Surina, B. 2017b: Phytosociological analysis of montane-subalpine dwarf willow shrub communities in the Julian Alps and on the Trnovski gozd plateau (NW and W Slovenia). Hacquetia 16 (2): 213-280.

Dakskobler, I., Čar, J., Terpin, R. \& Vončina, A. 2017a: Kranjski jeglič (Primula carniolica) $\mathrm{v}$ dveh robnih območjih občine Idrija in zakaj ga ni v Anderletovem seznamu flore Gorenjske. Proteus 79 (6): 254-260.

Dakskobler, I., Martinčičč, A. \& Rojšek, D. 2017b: New localities of Adiantum capillus-veneris in the river-basin of Volarja/Volarnik (the Julian Alps) and phytosociological analyses of its sites. Folia biologica et geologica 58 (1): 31-45.

Dakskobler, I. \& Martinčič, A. 2018: A new endemic plant community with Schoenus nigricans in the Southeastern Alps and northern Dinaric Alps. Folia biologica et geologica 59 (1): 5-28.

Dolinar, B., Vreš, B. \& Dakskobler, I. 2013: Pregled znanih in nova nahajališča kranjskega jegliča (Primula carniolica Jacq.) na Dolenjskem. Hladnikia 32: 3-21.

Dolinar, B., Vreš, B., Dakskobler, I., Schein, T. \& Stergaršek, J. 2015: Pregled nahajališč kranjskega jegliča (Primula carniolica Jacq.) v Notranjskem regijskem parku. Hladnikia 36: 31-45.

Dolinar, B., Dakskobler, I., Jogan, N. \& Vreš, B. 2017: Pregled nahajališč kranjskega jegliča (Primula carniolica Jacq.) v osrednjem delu njegovega areala. Hladnikia 39: 18-32.

Giovagnoli, L. \& Tasinazzo, S. 2012: Hymenostylio recurvirostriPinguiculetum poldinii ass. nova in the Valbrenta ravines (Venetian Prealps): a new palaeoendemic plant association belonging to the class Adiantetea Br.-Bl. 1948. Plant Sociology 29 (2): 49-58.

Hodgetts, N. et al. 2019: A miniature world in decline. European Red List of Mosses, Liverworts and Hornworts. Brussels, Belgium: IUCN. 87 pp.
Jogan, N., Kaligarič, M., Leskovar, I., Seliškar, A. \& Dobravec, J. 2004 Habitatni tipi Slovenije HTS 2004. Agencija Republike Slovenije za okolje, Ljubljana, 64 pp.

Maarel, van der E. 1979: Transformation of cover-abundance values in phytosociology and its effects on community similarity. Vegetatio 39 (2): $97-114$

Martinčič, A. 2016: Updated Red List of bryophytes of Slovenia. Hacquetia 15 (1): 107-126.

Martinčič, A., Wraber, T., Jogan, N., Podobnik, A., Turk, B., Vreš, B., Ravnik, V., Frajman, B., Strgulc Krajšek, S., Trčak, B., Bačič, T., Fischer, M. A., Eler, K. \& Surina, B. 2007: Mala flora Slovenije. Ključ za določanje praprotnic in semenk. Četrta, dopolnjena in spremenjena izdaja. Tehniška založba Slovenije, Ljubljana, 967 pp.

Mucina, L., Bultmann, H., Dierssen, K., Theurillat, J.-P., Raus, T. Čarni, A., Šumberová, K., Willner, W., Dengler, J., Garcia, R. G., Chytrý, M., Hájek, M., Di Pietro, R., Iakushenko, D., Pallas, J., Daniëls, F. J. A., Bergmeier, E., Santos Guerra, A., Ermakov, N., Valachovič, M., Schaminée, J. H. J., Lysenko, T., Didukh, Y. P., Pignatti, S., Rodwell, J. S., Capelo, J., Weber, H. E., Solomeshch, A., Dimopoulos, P., Aguiar, C., Hennekens, S. M. \& Tichý, L. 2016: Vegetation of Europe: hierarchical floristic classification system of vascular plant, bryophyte, lichen, and algal communities. Applied Vegetation Science 19: 3-264.

Pignatti, E. \& Pignatti, S. 2014: Plant Life of the Dolomites. Vegetation Structure and Ecology. Publication of the Museum of Nature South Tyrol Nr. 8, Naturmuseum Südtirol, Bozen, Springer Verlag, Heidelberg, 769 pp.

Pignatti, E. \& Pignatti, S. 2016: Plant Life of the Dolomites. Vegetation Tables. Publication of the Museum of Nature South Tyrol Nr. 11, Bozen, Springer Verlag, Heidelberg, 575 pp.

Podani, J. 2001: SYN-TAX 2000. Computer Programs for Data Analysis in Ecology and Systematics. User's Manual, Budapest, 53 pp.

Podobnik, A., Surina, B. \& Dakskobler, I. 2013: Zgodba o Bertolonijevi orlici v Sloveniji. Proteus 75 (7): 295-302.

Poldini, L. 1978: La vegetazione petrofila dei territori carsici nordadriatici. Spominski zbornik Maksa Wraberja 1905-1972. Poročila Vzhodnoalpsko-dinarskega društva za proučevanje vegetacije 14: 297-324, Ljubljana.

Ros, R. M., Mazimpaka, V., Abou-Salama, U., Aleffi, M., Blockeel, T. L., Brugués, M., Cano, M. J., Cros, R. M., Dia, M. G., Dirkse, G. M., El Saadawi, W., Erdağ, A., Ganeva, A., GonzálezMancebo, J. M., Hernstadt, I., Khalil, K., Kürschner, H., Lanfranco, E., Losada-Lima, A., Refai, M. S., Rodríguez-Nunez, S., Sabovljević, M., Sérgio, C., Shabbara, H., Sim-Sim, M. \& Söderström, M. 2007: Hepatics and Anthocerotes of the Mediterranean, an annotated checklist. Cryptogamie, Bryologie 28 (4): 351-437.

Ros, R. M., Mazimpaka, V., Abou-Salama, U., Aleffi, M., Blockeel, T. L., Brugués, M., Cros, R. M., Dia, M. G., Dirkse, G. M., Draper, I., El Saadawi, W., Erdağ, A., Ganeva, A., Gabriel, R., González-Mancebo, J. M., Hernstadt, I., Hugonnot, V., Khalil, K., Kürschner, H., Losada-Lima, A., Luís, L., Mifsud, S., Privitera, M., Puglisi, M., Sabovljević, M., Sérgio, C., Shabbara, H. M., SimSim, M., Sotiaux, A., Tacchi, R., Vanderpoorten, A. \& Werner, O. 2013: Mosses of the Mediterranean, an annotated checklist. Cryptogamie, Bryologie 34 (2): 99-283.

Rozman, A., Dakskobler, I. \& Šilc, U. 2020: Phytosociological analysis of basophilic Scots pine forests in the Southeastern Alps. Hacquetia 19 (1): 23-80. 
Seliškar, T., Vreš, B. \& Seliškar, A. 2003: FloVegSi 2.0. Fauna, Flora, Vegetation and Paleovegetation of Slovenia. Computer programme for arranging and analysis of biological data. Biološki inštitut ZRC SAZU, Ljubljana.

Surina, B. 2005: Subalpinska in alpinska vegetacija Krnskega pogorja v Julijskih Alpah. Scopolia 57: 1-122.

Surina, B. \& Dakskobler, I. 2005: Delimitation of the alliances Caricion firmae (Seslerietalia albicantis) and Seslerion juncifoliae (Seslerietalia juncifoliae) in the southeastern Alps and Dinaric mountains. Plant Biosystems 139 (3): 399-410.

Surina, B. \& Vreš, B. 2009: The association Drepanoclado uncinatiHeliospermetum pusilli (Arabidetalia caeruleae, Thlaspietea rotundifolii) in the Trnovski gozd plateau (Slovenia, NW Dinaric Mts).

Hacquetia 8 (1): 31-40

Surina, B. \& Dakskobler, I. 2013: Phytosociology and ecology of the Dinaric fir-beech forests (Omphalodo-Fagetum) at the northwestern part of the Illyrian floral province (NW Dinaric Alps). Hacquetia 12 (1): 11-85.

Šilc, U. \& Čarni, A. 2012: Conspectus of vegetation syntaxa in Slovenia. Hacquetia 11 (1): 113-164.

Theurillat, J.-P. 2004: Pflanzensoziologisches System. In: Aeschimann, D., Lauber, K., Moser D. M. \& Theurillat J.-P.: Flora alpina 3: Register. Haupt Verlag, Bern, Stuttgart, Wien, pp. 301-313.

Weber, H. E., Moravec, J. \& Theurillat, J. P. 2000: International Code of Phytosociological Nomenclature. 3rd. Edition. Journal of vegetation Science 11 (5): 739-766.

Wraber, T. 1986: Die Felsenmiere (Minuartia rupestris) - Vegetation der Julischen Alpen. Biološki vestnik 34 (1): 115-124.

\section{Appendix}

Figure 14: Dendrogram of relevés of rock crevice communities with Primula carniolica in the northwestern part of its ditribution area.

Slika 14: Dendrogram popisov združb skalnih razpok z vrsto Primula carniolica v severozahodnem delu njenega areala.

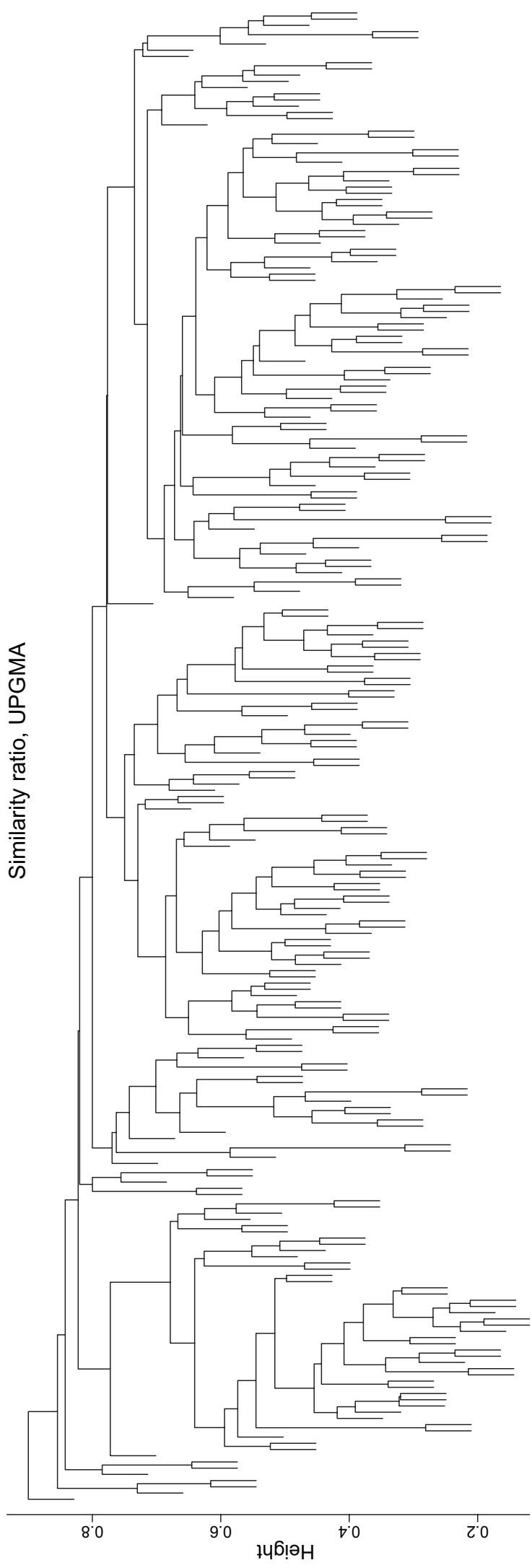


Table 1 (Tabela 1): Astrantio carniolicae-Primuletum carniolicae. Relevé number 1-43.

\section{Number of relevé (Zaporedna štev. popisa)}

Database number of relevé

(Delovna številka popisa)

Elevation in $\mathrm{m}$ (Nadmorska višina $\mathrm{v} \mathrm{m}$ )

Aspect (Lega)

Slope in degrees (Nagib v stopinjah)

Parent material (Matična podlaga)

Soil (Tla)

Stoniness in \% (Kamnitost v \%)

Cover of herb layer in \%

(Zastiranje zeliščne plasti v \%):

Cover of moss layer in \%

(Zastiranje mahovne plasti v \%)

Number of species (Število vrst)

Relevé area (Velikost popisne ploskve)

Date of taking relevé (Datum popisa)

Locality (Nahajališče)

Quadrant (Kvadrant)

Coordinate GK Y (D-48)

Coordinate GK X (D-48)

Diagnostic species of the association (Diagnostične vrste asociacije)

AP Primula carniolica

AP Palustriella commutata

AP Astrantia carniolica

AP Pinguicula alpina

AP Hymenostylium recurvirostre

PsSp Phyteuma scheuchzeri subsp. columnae

EP Molinia arundinacea

AP Valeriana saxatilis

ES Carexmucronata

Diagnostic species of the subassociation (Diagnostične vrste subasociacij)

\section{AP Paederotalutea}

AP Aster bellidiastrum

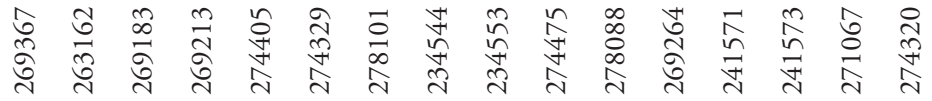

$450435435410600480435590700455540 \quad 590760750 \quad 310380$

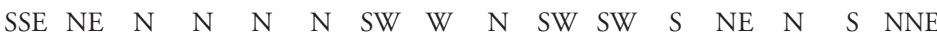
$\begin{array}{llllllllllllllll}90 & 90 & 90 & 80 & 80 & 85 & 80 & 80 & 95 & 90 & 90 & 70 & 80-100 & 80 & 90 & 80\end{array}$

$\begin{array}{llllllllllllllll}\mathrm{D} & \mathrm{D} & \mathrm{D} & \mathrm{D} & \mathrm{D} & \mathrm{D} & \mathrm{D} & \mathrm{D} & \mathrm{D} & \mathrm{D} & \mathrm{D} & \mathrm{D} & \mathrm{D} & \mathrm{D} & \mathrm{D} & \mathrm{D}\end{array}$

$\begin{array}{lllllllllllllllll}\mathrm{Li} & \mathrm{Li} & \mathrm{Li} & \mathrm{Li} & \mathrm{Li} & \mathrm{Li} & \mathrm{Li} & \mathrm{Li} & \mathrm{Li} & \mathrm{Li} & \mathrm{Li} & \mathrm{Li} & \mathrm{Li} & \mathrm{Li} & \mathrm{Li} & \mathrm{Li}\end{array}$ $\begin{array}{llllllllllllllll}100 & 100 & 100 & 100 & 100 & 100 & 100 & 100 & 100 & 100 & 100 & 100 & 60 & 100 & 100 & 100\end{array}$

$\begin{array}{lllllllllllllllll}\text { E1 } & 20 & 20 & 30 & 30 & 20 & 30 & 30 & 30 & 20 & 30 & 30 & 30 & 40 & 50 & 30 & 40\end{array}$

$\begin{array}{lllllllllllllllll}\text { E0 } & 20 & 20 & 20 & 40 & 20 & 20 & 10 & 20 & 20 & 20 & 30 & 20 & 30 & 30 & 10 & 50\end{array}$

$\begin{array}{llllllllllllllll}15 & 20 & 13 & 14 & 18 & 26 & 10 & 18 & 16 & 12 & 11 & 11 & 21 & 14 & 21 & 22\end{array}$ $\begin{array}{lllllllllllllllll}\mathrm{m}^{2} & 5 & 10 & 5 & 5 & 10 & 10 & 15 & 10 & 10 & 10 & 20 & 5 & 10 & 10 & 10 & 10\end{array}$

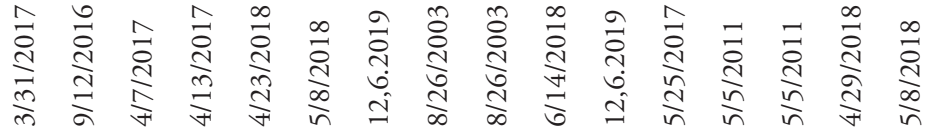

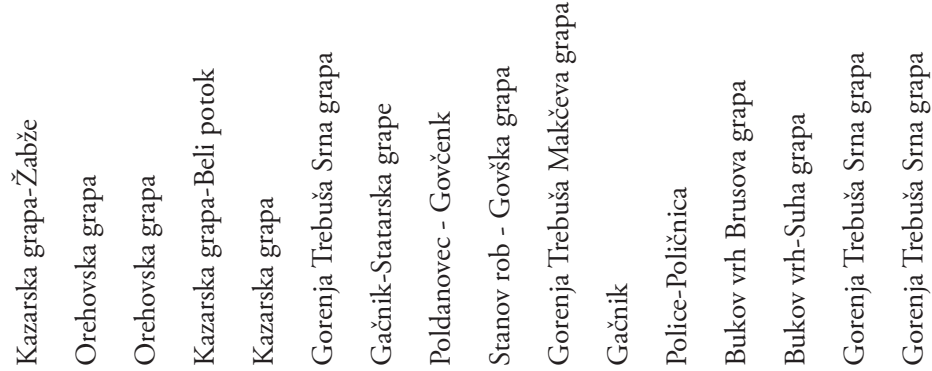

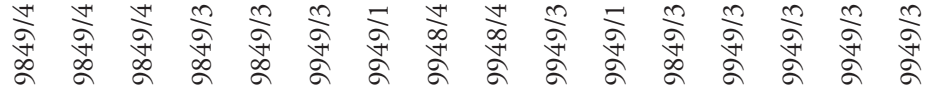

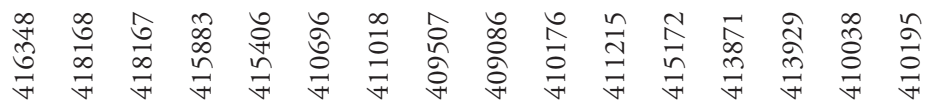

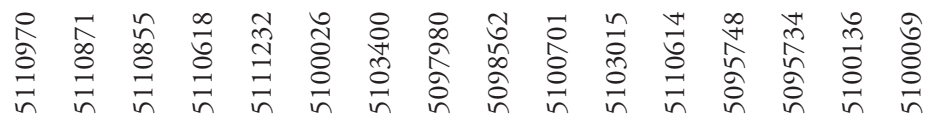




\section{\begin{tabular}{lllllllllllllllllllllllllll}
17 & 18 & 19 & 20 & 21 & 22 & 23 & 24 & 25 & 26 & 27 & 28 & 29 & 30 & 31 & 32 & 33 & 34 & 35 & 36 & 37 & 38 & 39 & 40 & 41 & 42 & 43 \\
\hline
\end{tabular}}

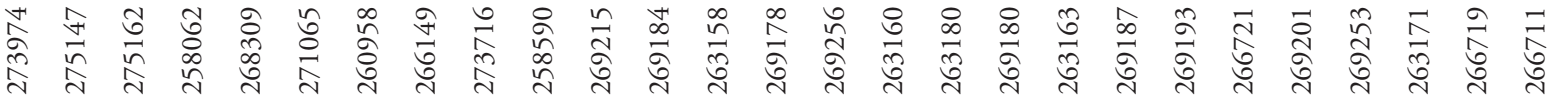
755430430230278270230255240480408435430425570430465430440475520440470560475440370

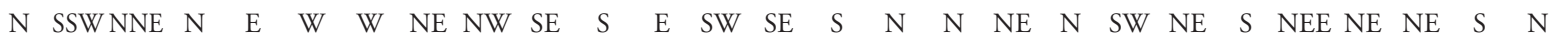
$\begin{array}{llllllllllllllllllllllllllll}85 & 90 & 90 & 95 & 80 & 85-95 & 95 & 80 & 95 & 80 & 85 & 90 & 80 & 85 & 85 & 80 & 80 & 85 & 90 & 90 & 90 & 85 & 80 & 80 & 90 & 80 & 90\end{array}$

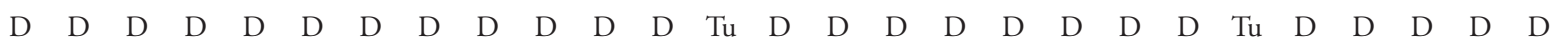

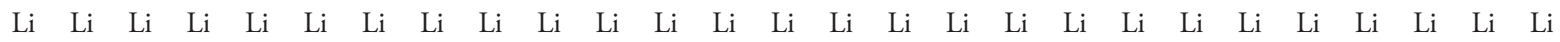

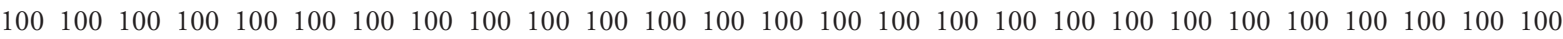
$\begin{array}{lllllllllllllllllllllllllll}30 & 30 & 20 & 30 & 30 & 30 & 30 & 30 & 25 & 30 & 30 & 60 & 40 & 30 & 35 & 30 & 40 & 30 & 60 & 40 & 20 & 40 & 30 & 30 & 30 & 40 & 30\end{array}$ $\begin{array}{lllllllllllllllllllllllllll}20 & 20 & 30 & 30 & 20 & 20 & 20 & 40 & 30 & 30 & 40 & 30 & 80 & 80 & 60 & 70 & 60 & 80 & 40 & 40 & 30 & 70 & 30 & 50 & 40 & 39 & 40\end{array}$ $\begin{array}{llllllllllllllllllllllllllll}16 & 27 & 24 & 32 & 17 & 17 & 26 & 12 & 27 & 15 & 21 & 23 & 10 & 8 & 13 & 13 & 14 & 14 & 15 & 14 & 14 & 11 & 10 & 15 & 13 & 19 & 12\end{array}$ $\begin{array}{llllllllllllllllllllllllllll}10 & 15 & 15 & 10 & 10 & 10 & 10 & 10 & 10 & 5 & 10 & 5 & 10 & 10 & 10 & 10 & 10 & 5 & 10 & 10 & 10 & 20 & 5 & 5 & 10 & 10 & 10\end{array}$

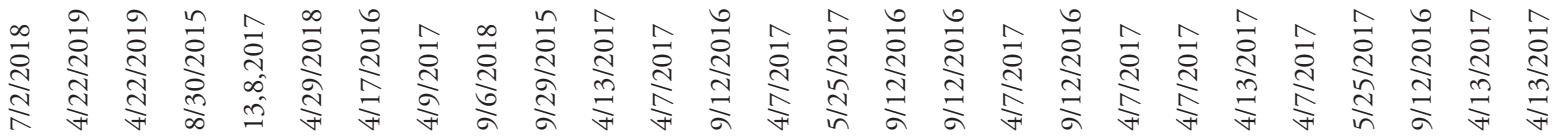

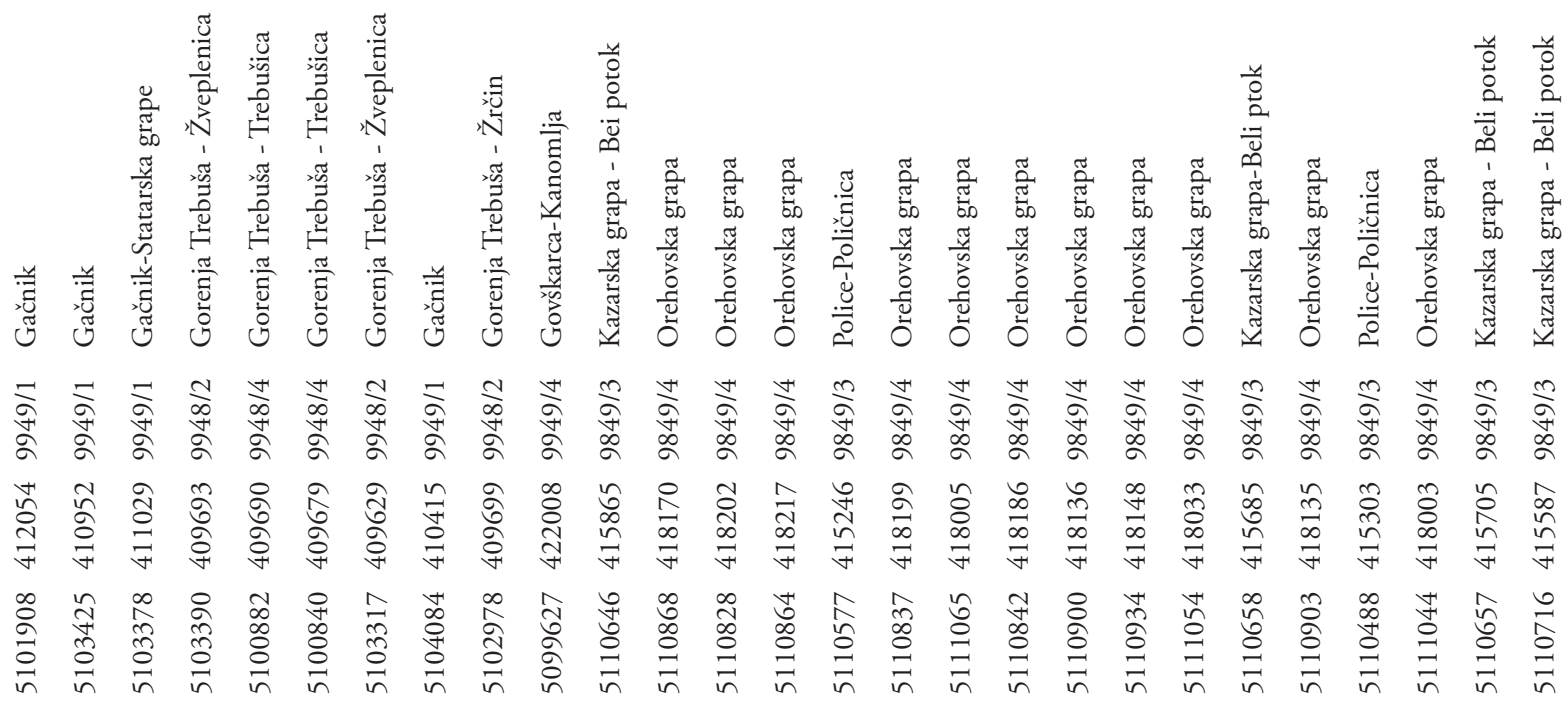

$\begin{array}{llllllllllllllllllllllllllllll}1 & 1 & 1 & 1 & 1 & 1 & 2 & 1 & + & 3 & 3 & 3 & + & 1 & + & 2 & 2 & 3 & 2 & 3 & 2 & 2 & 1 & 2 & 2 & 3 & 2 \\ 1 & 2 & 2 & + & + & . & 1 & 1 & 1 & 2 & 3 & 1 & 4 & 4 & 3 & 3 & 2 & 2 & 2 & 2 & 1 & 2 & 2 & 3 & 1 & 2 & 1 \\ + & + & + & 1 & + & 1 & + & . & + & \cdot & 1 & + & 3 & 1 & 3 & 2 & 2 & + & 1 & . & 1 & 2 & 1 & + & 2 & 2 & 2 \\ . & + & + & 2 & 2 & 1 & . & + & 1 & + & + & + & 1 & 1 & + & 3 & + & 1 & + & 1 & 2 & 2 & 2 & 3 & 3 & + & 2 & 3 \\ . & 1 & . & + & . & + & + & . & 1 & 2 & 1 & 1 & 4 & 4 & 4 & 4 & 3 & 4 & 3 & 3 & 3 & 3 & 2 & 2 & 2 & 1 & 3 \\ + & + & . & + & + & + & + & . & & + & \mathrm{r} & + & . & . & . & . & . & + & . & . & . & . & . & \mathrm{r} & . & \mathrm{r} & .\end{array}$

\begin{tabular}{llllllllll|}
1 & 1 & 2 & 1 & + & 1 & 2 & 1 & 1 & + \\
1 & + & + & 2 & 2 & + & 1 & 1 & + & + \\
\hline
\end{tabular}


AP Cystopteridion s. lat. (Astrantio-Paederotion luteae nom. prov.)

Orthothecium rufescens

Tofieldia calyculata

Aster bellidiastrum

Valeriana saxatilis

Carex brachystachys

Preissia quadrata

Asplenium viride

Valeriana tripteris

Fissidens dubius

Barbula crocea

Viola biflora

Eucladium verticillatum

Pellia endiviifolia

Soldanella minima

Moehringia muscosa

PcSp Physoplexido comosae-Saxifragion petraeae

Campanula cespitosa

Hieracium pospichalii

PC Potentilletalia caulescentis

Potentilla caulescens

Kernera saxatilis

AT Asplenietea trichomanis

Asplenium trichomanes

Asplenium ruta-muraria

TR Thlaspietea rotundifolii

Hieracium bifidum

Adenostyles glabra

Gymnocarpium robertianum

Achnatherum calamagrostis

Petasites paradoxus

Hieracium porrifolium

CD Caricetalia davallianae

Parnassia palustris

Schoenus nigricans

MC Montio-Cardaminetea

Conocephalum conicum

Campylium stellatum

Bryum pseudotriquetrum

Cratoneuron filicinum

Distichium capillaceum

Campylium calcareum

Campylium elodes

Ditrichum flexicaule

ES Elyno-Seslerietea

Sesleria caerulea

Phyteuma orbiculare

Carex ferruginea

Carex firma

Laserpitium peucedanoides

Betonica alopecuros
E0

E0

E0

E1

E0

E0

E1

E1

E1

E1

E1

E1

E1

E1

E1

E1

E1

E1

E1

E1

E1

E1

E0

E0

E0

E0

E0

E0

E0

E0

E1

E1

E1

E

E1

E1 


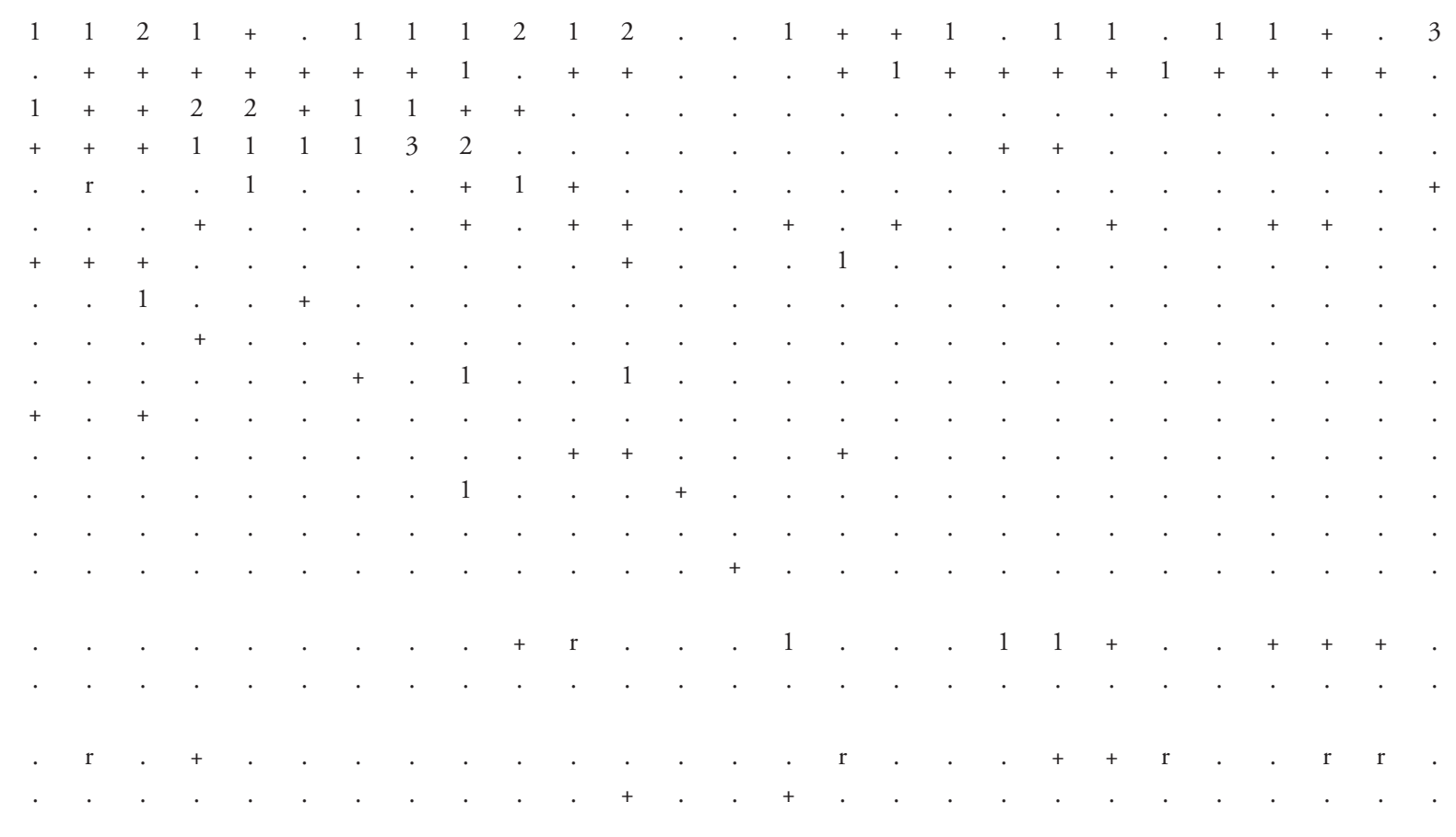


FB Festuco-Brometea

Buphthalmum salicifolium

Galium lucidum

E1

E1

CA Calthion

Caltha palustris

Cirsium oleraceum

E1

E1

BA Betulo-Alnetea

Salix appendiculata

$\mathrm{E} 2 \mathrm{a}$

Salix glabra

E1

MuA Mulgedio-Aconitetea

Chaerophyllum hirsutum

Crepis paludosa

E1

E1

EA Epilobietea angustifolii

Eupatorium cannabinum

E1

TG Trifolio-Geranietea

Laserpitium siler

Laserpitium latifolium

EP Erico-Pinetea

Calamagrostis varia

Erica carnea

Rhododendron hirsutum

Rhodothamnus chamaecistus

Carex ornithopoda

Aquilegia nigricans

Polygala chamaebuxus

Cirsium erisithales

E1

E1

E1

E1

E1

E1

E1

E1

E1

E1

VP Vaccinio-Piceetea

Veronica urticifolia

Solidago virgaurea

Aposeris foetida

Homogyne sylvestris

Oxalis acetosella

Picea abies

Gentiana asclepiadea

AF Aremonio-Fagion

Cyclamen purpurascens

Euphorbia carniolica

Primula vulgaris

Knautia drymeia

Cardamine trifolia

Helleborus niger

Potentilla carniolica

Anemone trifolia

Hemerocallis lilioasphodelus

Lamium orvala

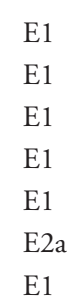

E1

TA Tilio-Acerion

Aruncus dioicus

Phyllitis scolopendrium

Polystichum aculeatum

Acer pseudoplatanus 
FS Fagetalia sylvaticae

Mycelis muralis

Salvia glutinosa

Carex umbrosa

Galeobdolon flavidum

Galium laevigatum

Brachypodium sylvaticum

Melica nutans

Prenanthes purpurea

Asarum europaeum subsp. caucasicum

Cardamine pentaphyllos

QP Quercetalia pubescenti-petraeae

Carex flacca

Fraxinus ornus

Ostrya carpinifolia

\section{E1}

E1

E1

E1

E1

E1

E1

Querco-Fagetea

Carex digitata

Hedera helix

Clematis vitalba

Hepatica nobilis

Taxus baccata

Frangula alnus

E1

E1

E1

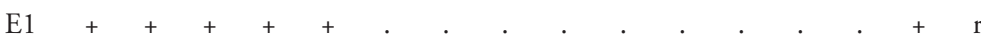

ML Mosses and lichens (Mahovi in lišaji)

Neckera crispa

E0

Ctenidium molluscum

E0

Tortella tortuosa

Heterocladium heteropterum

E0

Marchantia polymorpha

E0

E0

Mnium thomsonii

E0

Lophozia sp.

Brachythecium rutabulum

Dichodontium pellucidum

Bryum sp.

Encalypta vulgaris

Mnium marginatum

Rhynchostegium murale

E0

E0

E0

E0

E0

E0

E0 


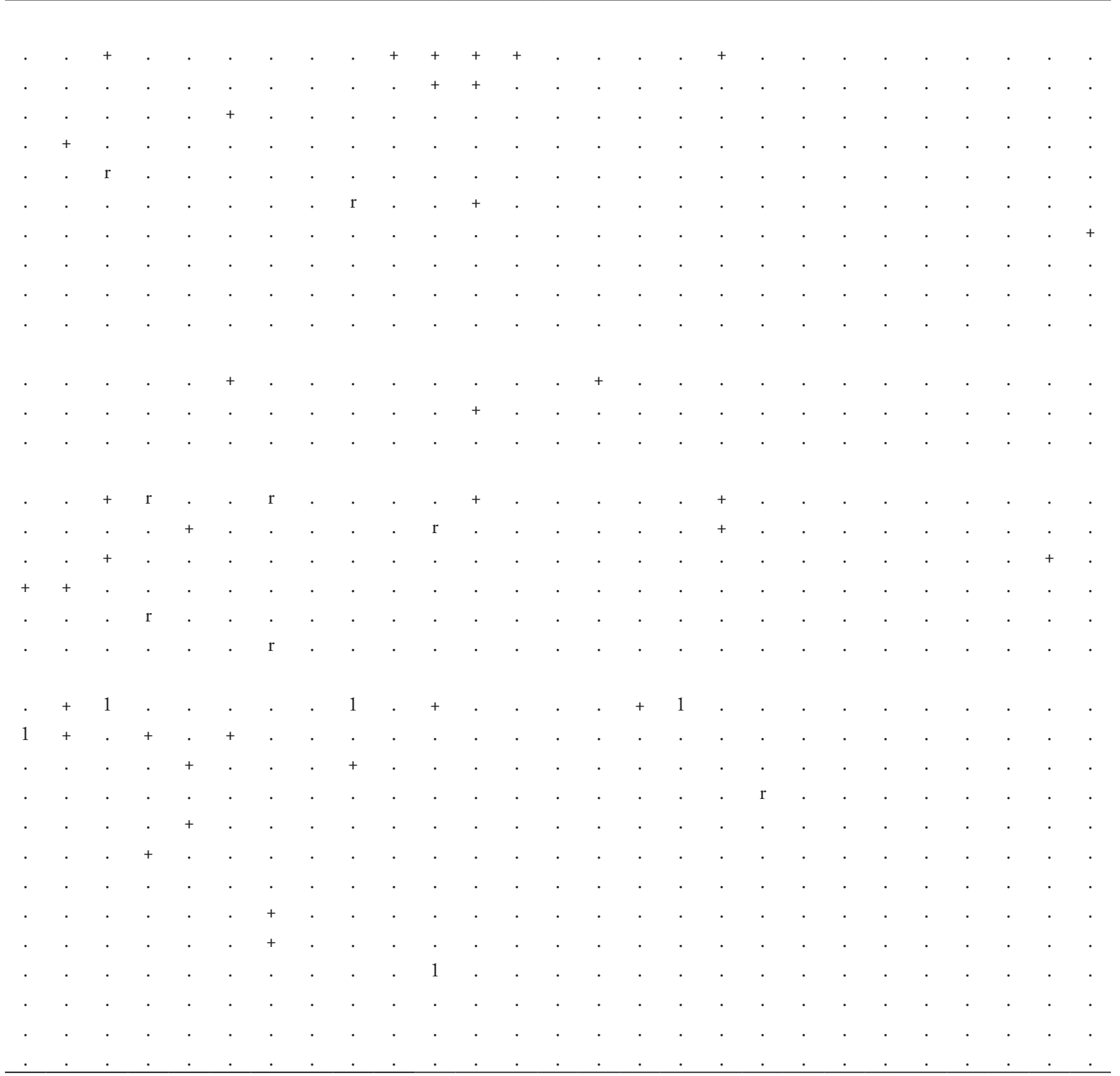


Table 1 (Tabela 1): Astrantio carniolicae-Primuletum carniolicae. Relevé number 44-56.

\section{Number of relevé (Zaporedna štev. popisa) \\ Database number of relevé \\ (Delovna številka popisa) \\ Elevation in $\mathrm{m}$ (Nadmorska višina v $\mathrm{m}$ ) \\ Aspect (Lega) \\ Slope in degrees (Nagib v stopinjah) \\ Parent material (Matična podlaga) \\ Soil (Tla) \\ Stoniness in \% (Kamnitost v \%) \\ Cover of herb layer in \% \\ (Zastiranje zeliščne plasti v \%): \\ Cover of moss layer in \% \\ (Zastiranje mahovne plasti v \%) \\ Number of species (Število vrst) \\ Relevé area (Velikost popisne ploskve)}

Date of taking relevé (Datum popisa)

Locality (Nahajališče)

Quadrant (Kvadrant)

Coordinate GK Y (D-48)

Coordinate GK X (D-48)

Diagnostic species of the association (Diagnostične vrste asociacije)

\section{$\begin{array}{lllllllllllllll}44 & 45 & 46 & 47 & 48 & 49 & 50 & 51 & 52 & 53 & 54 & 55 & 56 & \text { Pr. } & \text { Fr. }\end{array}$}

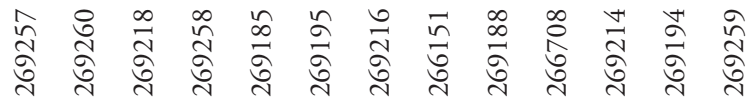

575580410580464520410260460525410520580

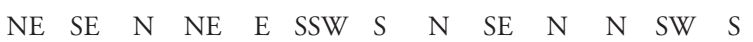

$\begin{array}{lllllllllllll}90 & 90 & 80 & 85 & 70 & 80 & 80 & 90 & 90 & 70 & 65 & 95 & 80\end{array}$

$\begin{array}{lllllllllllll}\mathrm{D} & \mathrm{D} & \mathrm{D} & \mathrm{D} & \mathrm{D} & \mathrm{D} & \mathrm{D} & \mathrm{D} & \mathrm{D} & \mathrm{D} & \mathrm{D} & \mathrm{D} & \mathrm{D}\end{array}$

$\begin{array}{lllllllllllll}\mathrm{Li} & \mathrm{Li} & \mathrm{Li} & \mathrm{Li} & \mathrm{Li} & \mathrm{Li} & \mathrm{Li} & \mathrm{Li} & \mathrm{Li} & \mathrm{Li} & \mathrm{Li} & \mathrm{Li} & \mathrm{Li}\end{array}$

$\begin{array}{lllllllllllll}100 & 100 & 100 & 100 & 70 & 100 & 100 & 100 & 100 & 100 & 100 & 100 & 100\end{array}$

$\begin{array}{llllllllllllll}\text { E1 } & 25 & 30 & 40 & 40 & 60 & 40 & 50 & 40 & 30 & 70 & 40 & 20 & 30\end{array}$

$\begin{array}{llllllllllllll}\text { E0 } & 65 & 30 & 50 & 40 & 30 & 60 & 80 & 30 & 40 & 30 & 50 & 20 & 30\end{array}$

$\begin{array}{lllllllllllll}12 & 16 & 17 & 16 & 15 & 23 & 20 & 7 & 7 & 23 & 22 & 17 & 13\end{array}$

$\begin{array}{llllllllllllll}\mathrm{m}^{2} & 10 & 10 & 10 & 20 & 5 & 5 & 10 & 10 & 5 & 10 & 10 & 5 & 5\end{array}$

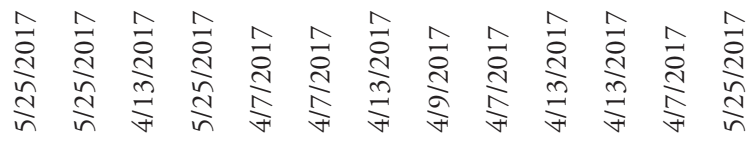

AP Primula carniolica

AP Palustriella commutata

AP Astrantia carniolica

AP Pinguicula alpina

AP Hymenostylium recurvirostre

PsSp Phyteuma scheuchzeri subsp. columnae

EP Molinia arundinacea

AP Valeriana saxatilis

ES Carex mucronata

\section{Diagnostic species of the subassociation (Diagnostične vrste subasociacij)}

AP Paederota lutea

AP Aster bellidiastrum

E1

E1

$\begin{array}{llllllllllllllll}\text { E1 } & + & 1 & 1 & 3 & 1 & 2 & + & 1 & 2 & 2 & 1 & 2 & 1 & 56 & 100 \\ \text { E0 } & 4 & 1 & 3 & 3 & 2 & 2 & 4 & 2 & 4 & . & 1 & . & . & 50 & 89 \\ \text { E1 } & 1 & 1 & 2 & 2 & 1 & 1 & 3 & . & + & + & 1 & . & 1 & 49 & 88 \\ \text { E1 } & 2 & 2 & 2 & 1 & 2 & 2 & 1 & 3 & 3 & 1 & 1 & 2 & 2 & 48 & 86 \\ \text { E0 } & 3 & 1 & + & 2 & + & 3 & 1 & . & . & 1 & 1 & 1 & 1 & 43 & 77 \\ \text { E1 } & . & + & . & . & . & . & + & . & + & . & . & . & . & 27 & 48 \\ \text { E1 } & . & + & . & 1 & 1 & + & . & . & . & 1 & + & . & + & 24 & 43 \\ \text { E1 } & . & . & + & 1 & . & . & . & + & . & + & + & + & + & 24 & 43 \\ \text { E1 } & . & 1 & + & + & . & + & . & . & . & + & + & 1 & 2 & 21 & 38\end{array}$


AP Cystopteridion s. lat. (Astrantio-Paederotion luteae nom. prov.)

Orthothecium rufescens

Tofieldia calyculata

Aster bellidiastrum

Valeriana saxatilis

Carex brachystachys

Preissia quadrata

Asplenium viride

Valeriana tripteris

Fissidens dubius

Barbula crocea

Viola biflora

Eucladium verticillatum

Pellia endiviifolia

Soldanella minima

Moehringia muscosa

PcSp Physoplexido comosae-Saxifragion petraeae

Campanula cespitosa

Hieracium pospichalii

PC Potentilletalia caulescentis

Potentilla caulescens

Kernera saxatilis

AT Asplenietea trichomanis

Asplenium trichomanes

Asplenium ruta-muraria

TR Thlaspietea rotundifolii

Hieracium bifidum

Adenostyles glabra

Gymnocarpium robertianum

Achnatherum calamagrostis

Petasites paradoxus

Hieracium porrifolium

CD Caricetalia davallianae

Parnassia palustris

Schoenus nigricans

MC Montio-Cardaminetea

Conocephalum conicum

Campylium stellatum

Bryum pseudotriquetrum

Cratoneuron filicinum

Distichium capillaceum

Campylium calcareum

Campylium elodes

Ditrichum flexicaule

ES Elyno-Seslerietea

Sesleria caerulea

Phyteuma orbiculare

Carex ferruginea

Carex firma

Laserpitium peucedanoides

Betonica alopecuros

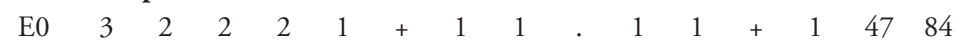

$\mathrm{E} 1+++.+2 .+1.25054$

$\mathrm{E} 1 . .5 . \quad . \quad . \quad . \quad 1.2443$

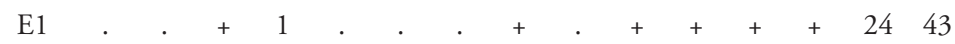

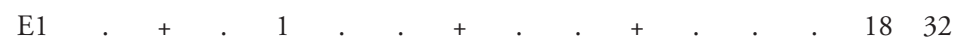

$\mathrm{E} 0 .++.+2 .+1.2929$

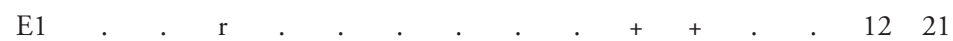

$\mathrm{E} 1.5 . \quad . \quad . \quad . \quad . \quad . \quad . \quad .6611$

E0 . . . . . . . . . ..+59

$\mathrm{E} 0$. . . . . . . . . . . 1.54

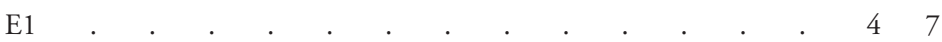

$\mathrm{E} 0$. . . . . . . . . . . . . . . 47

$\mathrm{E} 0$. . . . . . . . . . . . . . 35

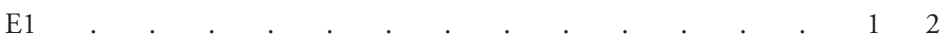

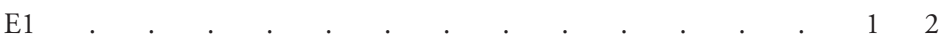

$\mathrm{E} 1++++.+.+. \quad . \quad . \quad 1 \quad 1527$

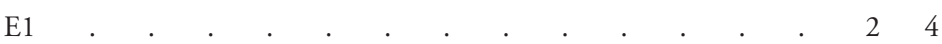

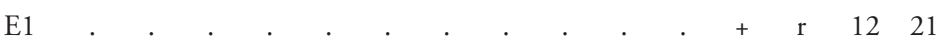

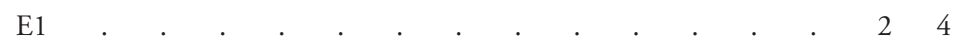

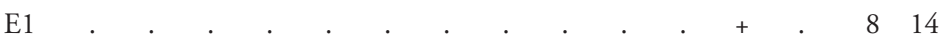

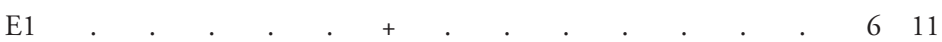

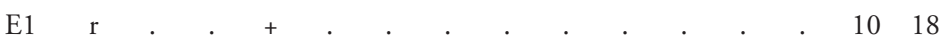

$\mathrm{E} 1$. . . . . . . . . . . . . . 7413

$\mathrm{E} 1$. . . . . . . . . . . . 59

$\mathrm{E} 1 . .5 . \quad . \quad . \quad . \quad . \quad . \quad . \quad . \quad 112$

$\mathrm{E} 1 . .5 . \quad . \quad . \quad . \quad . \quad . \quad . \quad 112$

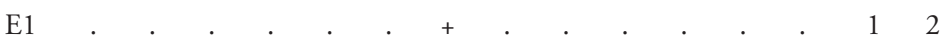

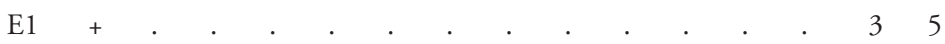

$\mathrm{E} 1 . .5 . \quad . \quad . \quad . \quad . \quad . \quad 35$

$\mathrm{E} 0$. . . . . . . . . . 1832

$\mathrm{E} 0$. . . . . . . . . 4 . 4 . 4

$\mathrm{E} 0$. . . . . . . . . . . 244

$\mathrm{E} 0$. . . . . . . . . . . . 244

$\mathrm{E} 0$. . . . . . . . . . . . 112

$\mathrm{E} 0$. . . . . . . . . . . 1 . . 112

$\mathrm{E} 0$. . . . . . . . . . . . . 412

$\mathrm{E} 0$. . . . . . . . . . . 12

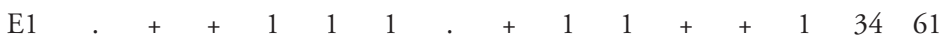

$\mathrm{E} 1 .+.+2 .+.+59$

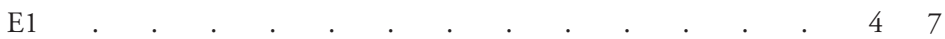

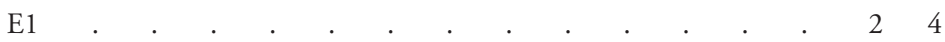

$\mathrm{E} 1$. . . . . . . . . . . . . . . 12

E1 . . . . . . . . . . . 112 
FB Festuco-Brometea

Buphthalmum salicifolium

Galium lucidum

E1

E1

CA Calthion

Caltha palustris

Cirsium oleraceum

E1

E1

BA Betulo-Alnetea

Salix appendiculata

Salix glabra

E2a

MuA Mulgedio-Aconitetea

Chaerophyllum hirsutum

Crepis paludosa

E1

E1

EA Epilobietea angustifolii

Eupatorium cannabinum

E1

TG Trifolio-Geranietea

Laserpitium siler

Laserpitium latifolium

EP Erico-Pinetea

Calamagrostis varia

Erica carnea

Rhododendron hirsutum

Rhodothamnus chamaecistus

Carex ornithopoda

Aquilegia nigricans

Polygala chamaebuxus

Cirsium erisithales

E1

E1

E

$\mathrm{E} 1++.+2 .+.+2 .+1832$

E1

E1

E1

E1

E1

E1

E1

VP Vaccinio-Piceetea

Veronica urticifolia

E1

Solidago virgaurea

Aposeris foetida

Homogyne sylvestris

Oxalis acetosella

Picea abies

Gentiana asclepiadea

AF Aremonio-Fagion

Cyclamen purpurascens

Euphorbia carniolica

Primula vulgaris

Knautia drymeia

Cardamine trifolia

Helleborus niger

Potentilla carniolica

Anemone trifolia

Hemerocallis lilioasphodelus

Lamium orvala

TA Tilio-Acerion

Aruncus dioicus

Phyllitis scolopendrium

Polystichum aculeatum

Acer pseudoplatanus 
FS Fagetalia sylvaticae

Mycelis muralis

E1

Salvia glutinosa

Carex umbrosa

Galeobdolon flavidum

Galium laevigatum

Brachypodium sylvaticum

Melica nutans

Prenanthes purpurea

Asarum europaeum subsp. caucasicum

Cardamine pentaphyllos

QP Quercetalia pubescenti-petraeae

Carex flacca

Fraxinus ornus

E1

Ostrya carpinifolia

QF Querco-Fagetea

Carex digitata

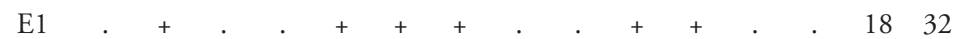

Hedera helix

E1

Clematis vitalba

E1

Hepatica nobilis

Taxus baccata

Frangula alnus

ML Mosses and lichens (Mahovi in lišaji)

Neckera crispa

Ctenidium molluscum

Tortella tortuosa

Heterocladium heteropterum

Marchantia polymorpha

Mnium thomsonii

Lophozia sp.

Brachythecium rutabulum

Dichodontium pellucidum

Bryum sp.

Encalypta vulgaris

Mnium marginatum

Rhynchostegium murale

\section{Legend / Legenda}

D Dolomite - dolomit

Tu Tufa - lehnjak

Li Lithosol - kamnišče

Pr. Presence (number of relevés in which the species is presented) - število popisov, v katerih se pojavlja vrsta

Fr. Frequency in $\%-$ frekvenca $\mathrm{\%} \%$ 


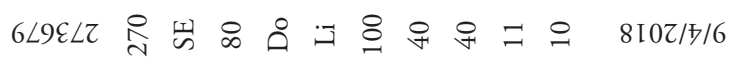

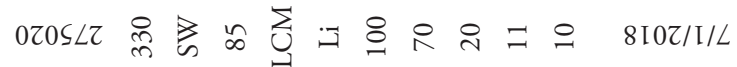
E9ISLr \&

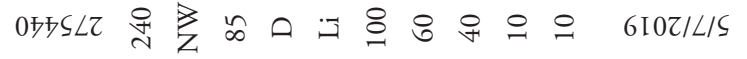

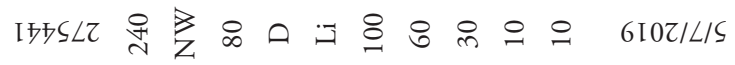

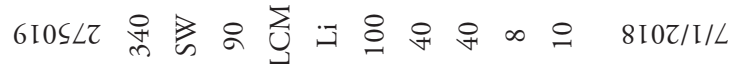

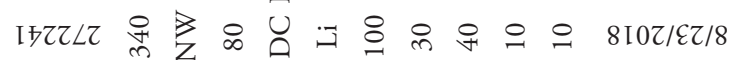

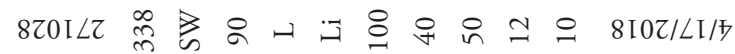

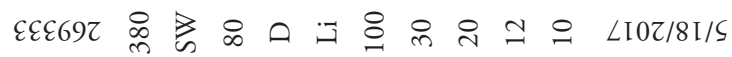
I89ELr 8850દz

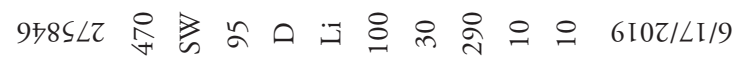
$\angle 8 \mathrm{IS} L \tau$ 㫕艺 \&

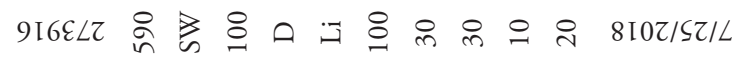

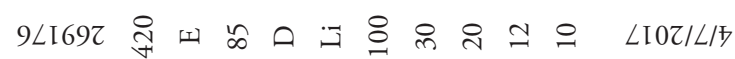

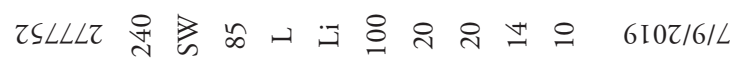

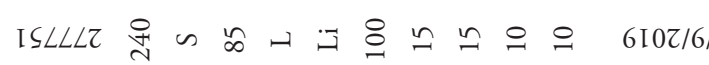

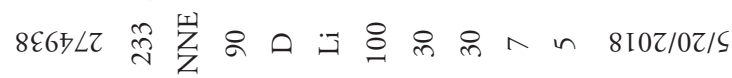

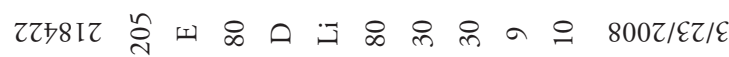

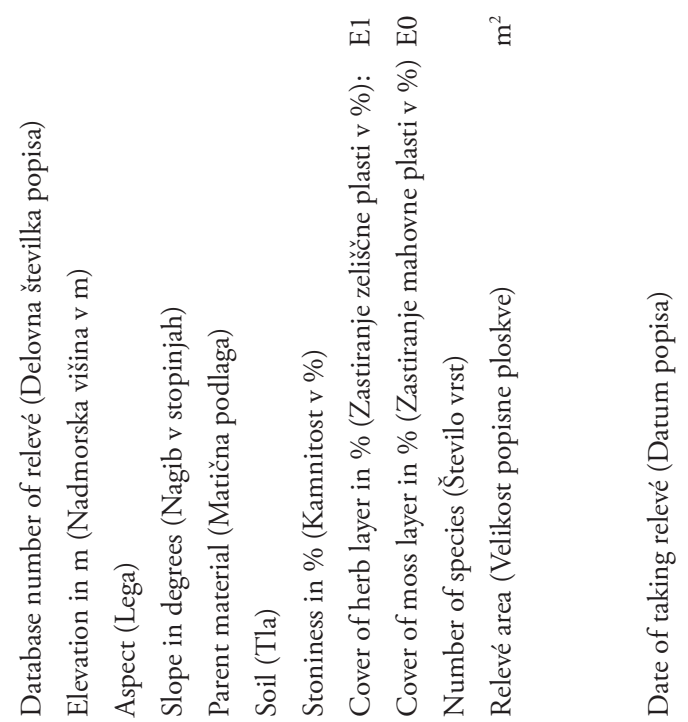

\begin{tabular}{|c|c|c|c|}
\hline 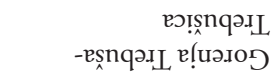 & ๓/8ゅ66 & $80 \angle 607$ & 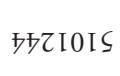 \\
\hline !ฺn & I/6ゅ86 & SEG0 I† & 6999IIS \\
\hline 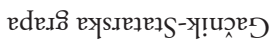 & I/65 66 & 00圠㠺 & $9 \amalg \amalg \mathcal{E} 0$ IS \\
\hline 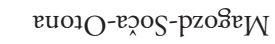 & $I / \angle 5 \angle 6$ & $26506 \mathcal{E}$ & 0Eゅ9ZIS \\
\hline 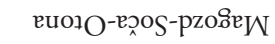 & $I / \angle \mapsto \angle 6$ & E6ワ06ع & 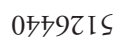 \\
\hline !ฺn ә әу! & I/6甲86 & SS80I五 & †LL9IIS \\
\hline 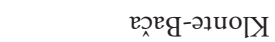 & I/6ゅ86 & I66甲㠺 & $9 \angle \angle E$ IIS \\
\hline 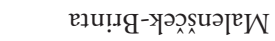 & ந/Lゅ $\angle 6$ & ऽ $0 \varpi 96 \mathcal{E}$ & $0 \angle L 0 Z I S$ \\
\hline уә્्र..qe & $\varepsilon / 6 \varpi 86$ & SI9サI五 & SカEG0IS \\
\hline 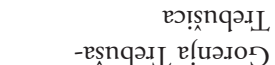 & て/8†66 & $8 \varepsilon 9607$ & ZSगI0IS \\
\hline ер्रॅg-леun & I/6586 & Ð6ES IT & E96EIIS \\
\hline 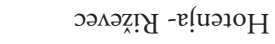 & $1 / 6566$ & 068E泫 & $\mathcal{E} \varsigma \mathcal{E} \mathcal{E}_{0}$ IS \\
\hline 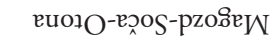 & $I / \angle y \angle G$ & $\varsigma_{0} \varsigma_{06 \mathcal{E}}$ & L6e9ZIS \\
\hline 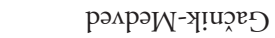 & I/6甲 66 & I6IIIJ & EE8Z0IS \\
\hline 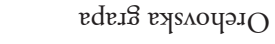 & †/6ゅ86 & 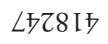 & 0I80IIS \\
\hline 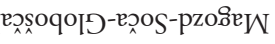 & $I / \angle \Phi \angle G$ & $8 ゅ 506 \mathcal{E}$ & 0099ZIS \\
\hline 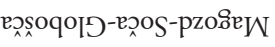 & $I / \angle \Phi \angle G$ & I⿰七06 & ¥099ZIS \\
\hline вұ!. & $\varepsilon / 87 L 6$ & 乙E乙E0历 & 9908IIS \\
\hline 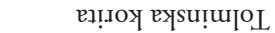 & I/8ゅ86 & OSZE0T & Z†08IIS \\
\hline
\end{tabular}




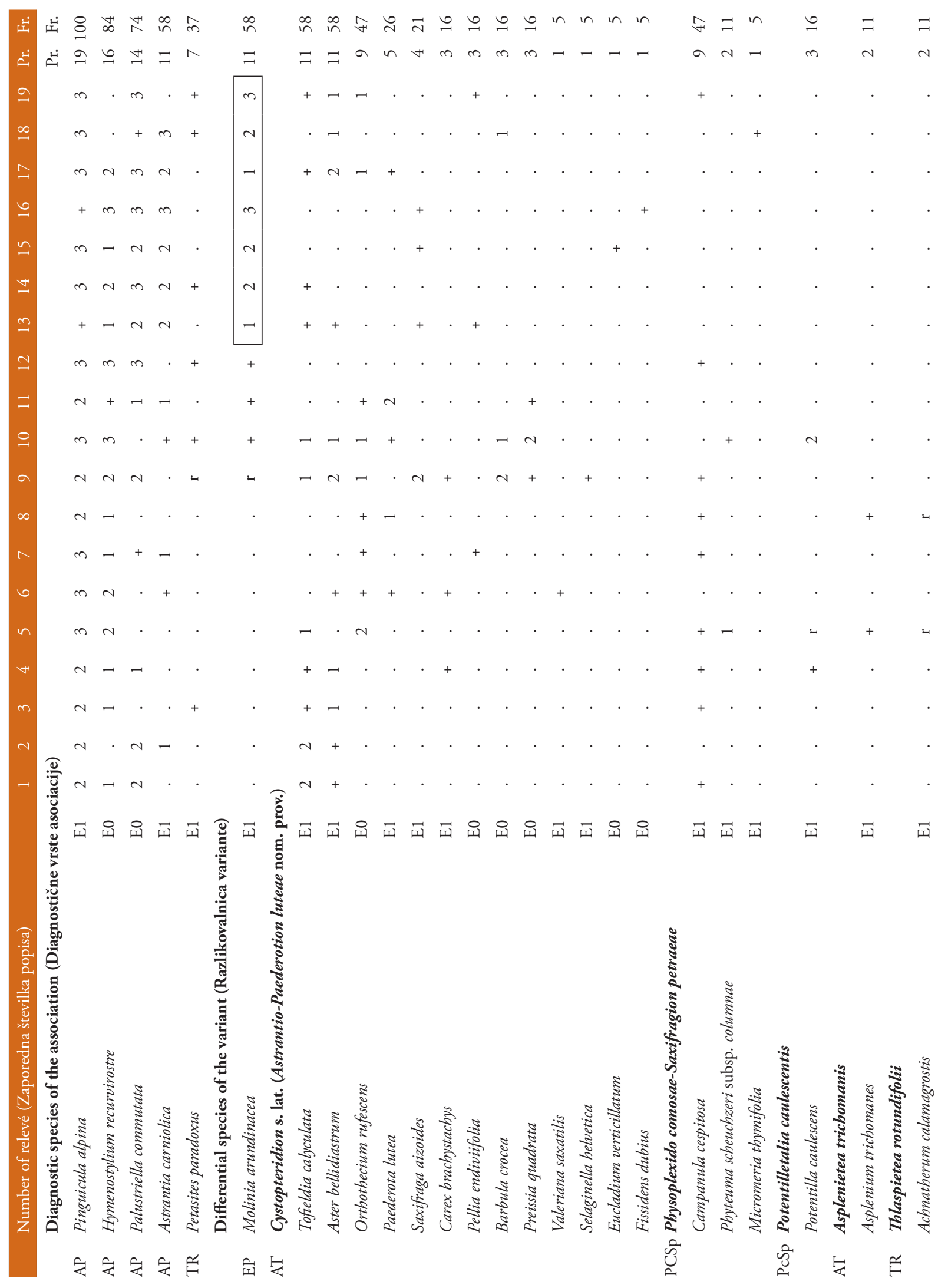




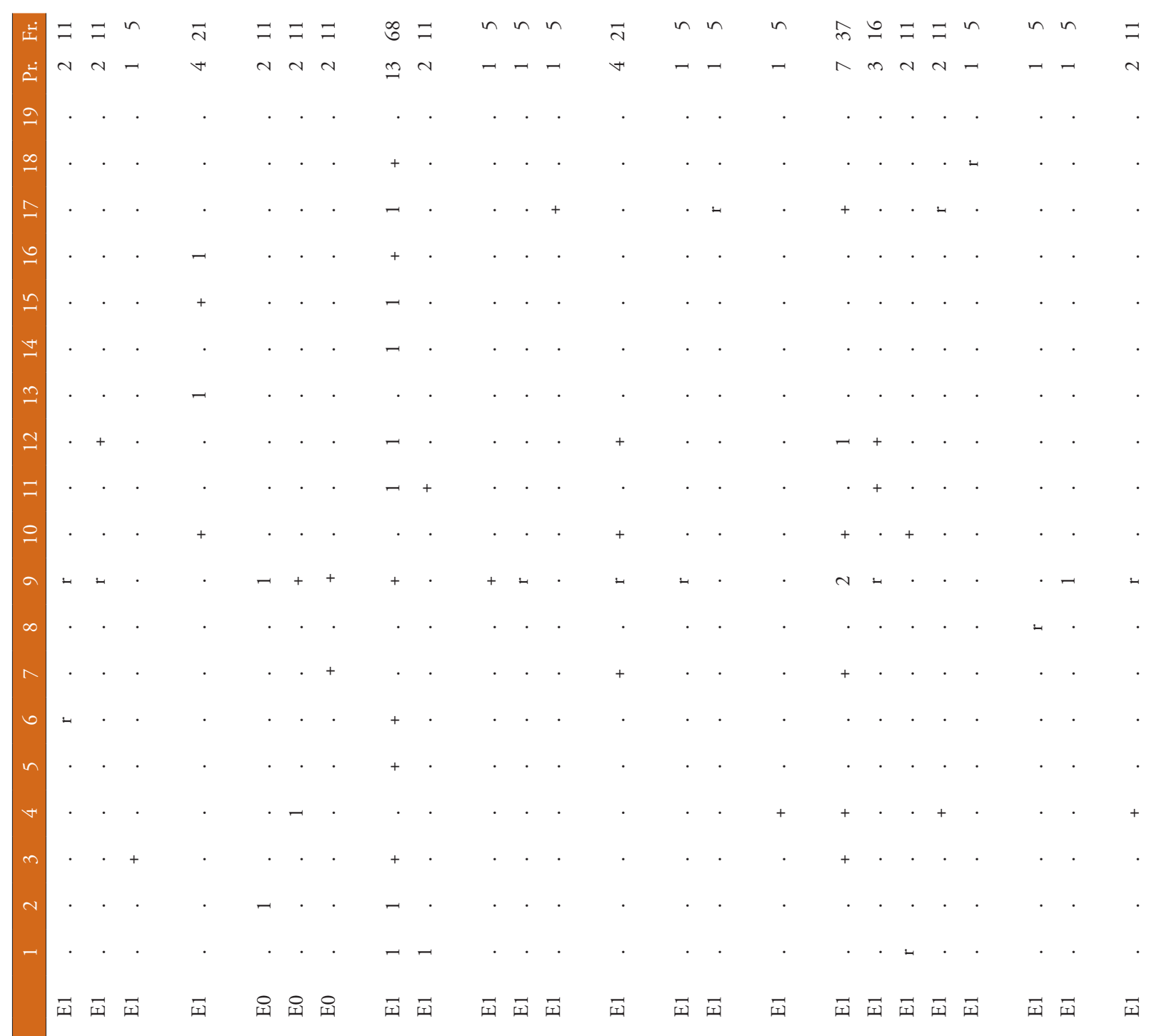




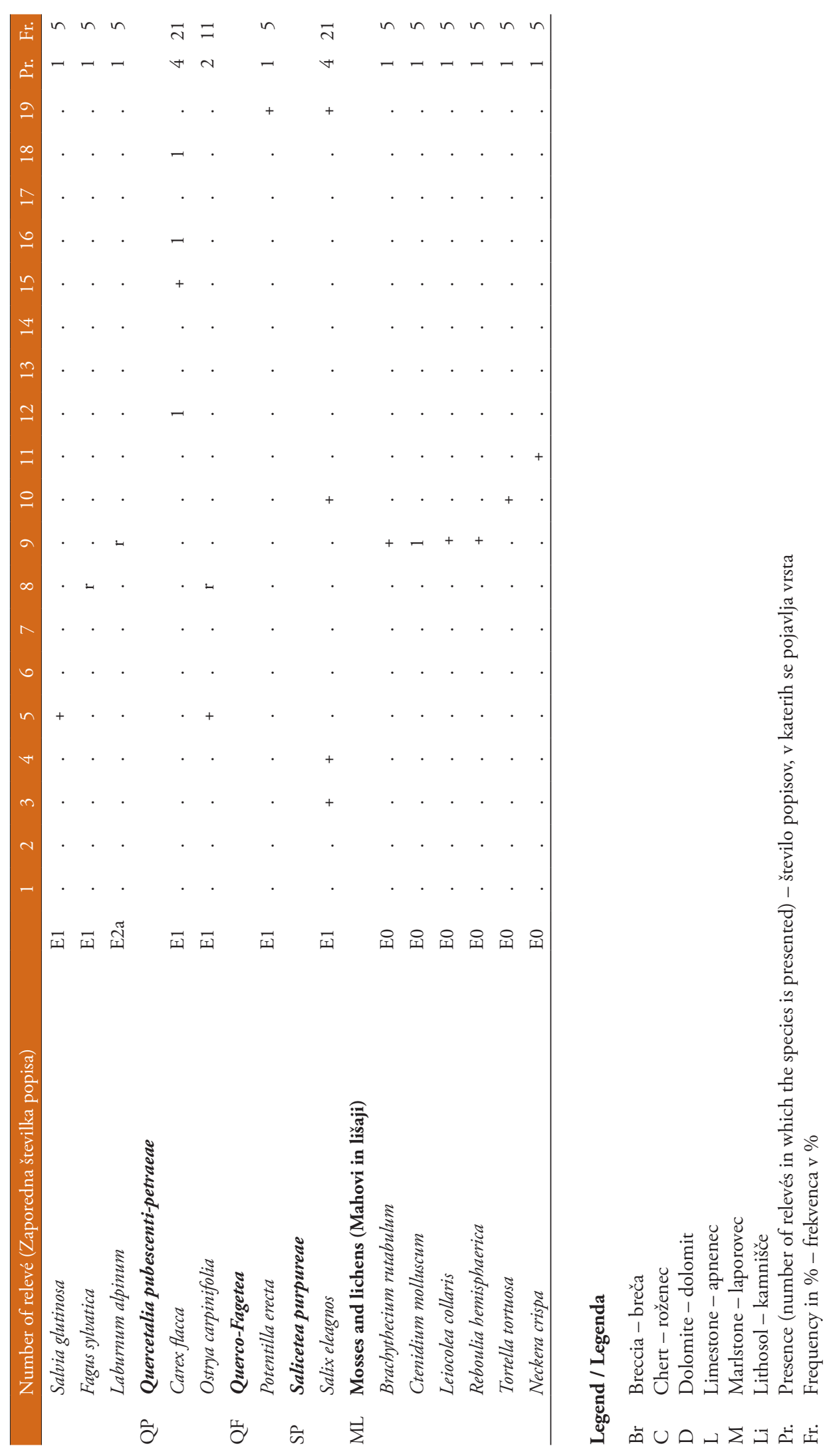


Table 3 (Tabela 3): Campanulo cespitosae-Saxifragetum aizoidis.

\begin{tabular}{|c|c|c|c|c|c|c|c|c|c|c|c|c|c|c|c|}
\hline Number of relevé (Zaporedna štev. popis & & 1 & 2 & 3 & 4 & 5 & 6 & 7 & 8 & 9 & 10 & 11 & 12 & 13 & 14 \\
\hline $\begin{array}{l}\text { Database number of relevé } \\
\text { (Delovna številka popisa) }\end{array}$ & & 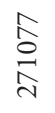 & $\begin{array}{l}\stackrel{\infty}{\hat{0}} \\
\stackrel{\lambda}{ત}\end{array}$ & 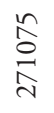 & $\begin{array}{l}\vec{\sigma} \\
\underset{N}{N}\end{array}$ & 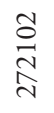 & $\begin{array}{l}\stackrel{n}{\hat{n}} \\
\stackrel{N}{N}\end{array}$ & $\begin{array}{l}\stackrel{0}{N} \\
\underset{N}{N}\end{array}$ & $\underset{\stackrel{N}{N}}{\stackrel{N}{N}}$ & 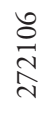 & 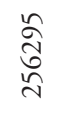 & 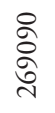 & 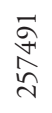 & 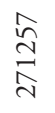 & $\underset{\stackrel{N}{\sim}}{\stackrel{\sim}{N}}$ \\
\hline Elevation in $\mathrm{m}$ (Nadmorska višina $\mathrm{v} \mathrm{m})$ & & ஓ & ஓ & ๙ิ & $\stackrel{Ð}{\forall}$ & $\underset{\sim}{\sim}$ & i & $\stackrel{n}{n}$ & $\hat{\approx}$ & 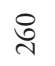 & $\widehat{\approx}$ & ๙ & $\stackrel{n}{n}$ & $\stackrel{\underset{f}{+}}{ }$ & 용 \\
\hline Aspect (Lega) & & $\stackrel{\sim}{\mathscr{N}}$ & 岀 & 8 & 忞 & 㭊 & 刿 & 島 & $\tilde{\omega}$ & 島 & 㥈 & Z & 空 & 崩 & 디 \\
\hline Slope in degrees (Nagib v stopinjah) & & 70 & 70 & 85 & 80 & 85 & 90 & 85 & 85 & 80 & 100 & 80 & 80 & 85 & 80 \\
\hline Parent material (Matična podlaga) & & $\sum_{\leftrightharpoons}^{\bigcup}$ & $\sum_{\lrcorner}^{\cup}$ & $\sum_{\leftrightharpoons}^{\bigcup}$ & $\sum_{\leftrightharpoons}^{\bigcup}$ & a & U & U & $\breve{\varrho}$ & U & $\varangle$ & $\underset{\psi}{U}$ & $\varangle$ & $\sum_{\leftrightharpoons}^{\bigcup}$ & $\sum_{\leftrightharpoons}^{\bigcup}$ \\
\hline Soil (Tla) & & $\mathrm{Li}$ & $\mathrm{Li}$ & $\mathrm{Li}$ & $\mathrm{Li}$ & $\mathrm{Li}$ & $\mathrm{Li}$ & $\mathrm{Li}$ & $\mathrm{Li}$ & $\mathrm{Li}$ & $\mathrm{Li}$ & $\mathrm{Li}$ & $\mathrm{Li}$ & $\mathrm{Li}$ & $\mathrm{Li}$ \\
\hline Stoniness in \% (Kamnitost v \%) & & 80 & 80 & 80 & 80 & 80 & 90 & 80 & 80 & 70 & 100 & 80 & 80 & 70 & 70 \\
\hline $\begin{array}{l}\text { Cover of herb layer in \% } \\
\quad \text { (Zastiranje zeliščne plasti v \%): }\end{array}$ & E1 & 40 & 40 & 30 & 40 & 30 & 20 & 40 & 40 & 50 & 15 & 30 & 30 & 50 & 50 \\
\hline $\begin{array}{l}\text { Cover of moss layer in } \% \\
\quad(\text { Zastiranje mahovne plasti v \%) }\end{array}$ & E0 & 40 & 10 & 40 & 40 & 30 & 25 & 39 & 30 & 20 & 10 & 20 & 20 & 50 & 30 \\
\hline Number of species (Število vrst) & & 15 & 10 & 12 & 25 & 18 & 18 & 15 & 13 & 20 & 11 & 11 & 18 & 25 & 26 \\
\hline Relevé area (Velikost popisne ploskve) & $\mathrm{m}^{2}$ & 10 & 10 & 10 & 10 & 10 & 10 & 10 & 10 & 10 & 5 & 10 & 10 & 10 & 10 \\
\hline Date of taking relevé (Datum popisa) & & 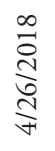 & 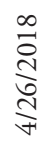 & 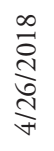 & 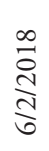 & 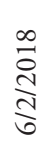 & 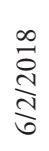 & $\frac{\infty}{\stackrel{\infty}{a}} \underset{\frac{\infty}{n}}{\frac{\infty}{n}}$ & $\frac{\infty}{\stackrel{\infty}{a}} \underset{\frac{N}{n}}{\frac{\infty}{\infty}}$ & $\frac{\infty}{\stackrel{\infty}{a}}$ & 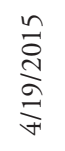 & 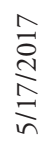 & 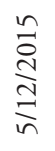 & 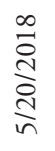 & 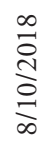 \\
\hline Locality (Nahajališče) & & 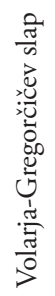 & 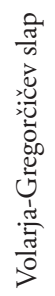 & 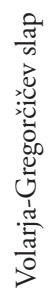 & 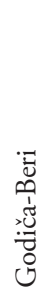 & 苞 & 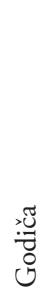 & 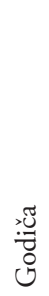 & 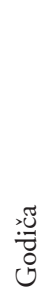 & 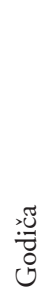 & 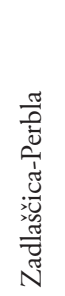 & 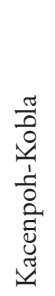 & 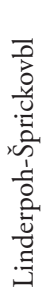 & 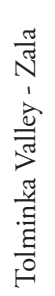 & 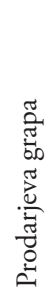 \\
\hline Quadrant (Kvadrant) & & $\frac{\stackrel{+}{~}}{\stackrel{+}{\sigma}}$ & 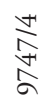 & 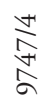 & $\begin{array}{l}\stackrel{N}{\infty} \\
\stackrel{+}{+} \\
\stackrel{\infty}{\alpha}\end{array}$ & $\begin{array}{l}\stackrel{N}{\infty} \\
\stackrel{\infty}{+} \\
\infty \\
\sigma\end{array}$ & $\begin{array}{l}\stackrel{1}{\infty} \\
\stackrel{\infty}{+} \\
\stackrel{\infty}{\alpha}\end{array}$ & $\begin{array}{l}\stackrel{N}{\infty} \\
\stackrel{\infty}{+} \\
\stackrel{\infty}{\alpha}\end{array}$ & $\begin{array}{l}\stackrel{N}{\infty} \\
\stackrel{\infty}{+} \\
\stackrel{\infty}{\alpha}\end{array}$ & $\begin{array}{l}\stackrel{N}{\infty} \\
\stackrel{\infty}{+} \\
\stackrel{\infty}{\alpha}\end{array}$ & $\begin{array}{l}\stackrel{+}{\infty} \\
\stackrel{\infty}{+} \\
\stackrel{+}{\sigma}\end{array}$ & $\frac{\stackrel{+}{a}}{\stackrel{+}{a}}$ & 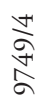 & $\begin{array}{l}\frac{n}{\infty} \\
\stackrel{0}{+} \\
\stackrel{\alpha}{\alpha}\end{array}$ & 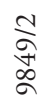 \\
\hline Coordinate GK Y (D-48) & $\mathrm{m}$ & 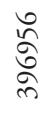 & 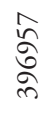 & $\begin{array}{l}\overrightarrow{8} \\
\stackrel{\circ}{\circ} \\
\text { ๙ }\end{array}$ & 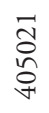 & 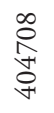 & 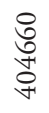 & $\begin{array}{l}\overrightarrow{\widehat{O}} \\
\text { †े } \\
\stackrel{+}{ }\end{array}$ & \begin{tabular}{l} 
o \\
\multirow{\mho}{0}{} \\
\multirow{+}{*}{} \\
$\stackrel{+}{+}$
\end{tabular} & 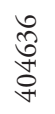 & 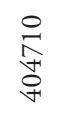 & $\begin{array}{l}\stackrel{\infty}{\underset{\sigma}{\sigma}} \\
\stackrel{+}{+}\end{array}$ & $\begin{array}{l}\frac{\infty}{\infty} \\
\stackrel{\infty}{\forall}\end{array}$ & 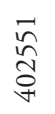 & 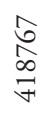 \\
\hline Coordinate GK X (D-48) & $\mathrm{m}$ & 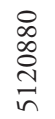 & 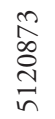 & 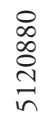 & $\begin{array}{l}\text { D } \\
\stackrel{\sim}{\sigma} \\
\cdots \\
\bar{n}\end{array}$ & $\begin{array}{l}0 \\
\stackrel{0}{0} \\
0 \\
\vec{n} \\
n\end{array}$ & $\begin{array}{l}0 \\
0 \\
0 \\
\Xi \\
\bar{n}\end{array}$ & $\begin{array}{l}\stackrel{n}{+} \\
\stackrel{0}{0} \\
\stackrel{్}{n}\end{array}$ & 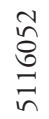 & 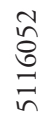 & $\begin{array}{l}\stackrel{\infty}{\infty} \\
\stackrel{2}{\approx} \\
\stackrel{\vec{n}}{n}\end{array}$ & $\frac{\underset{n}{n}}{\stackrel{\varpi}{\sim}}$ & 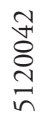 & $\begin{array}{l}\stackrel{\infty}{\approx} \\
\stackrel{\approx}{\triangleq}\end{array}$ & 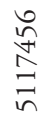 \\
\hline
\end{tabular}

AP Saxifraga aizoides

AP Palustriella commutata

PcSp Campanula cespitosa

EP Molinia arundinacea

EP Calamagrostis varia

AP Preissia quadrata

AP Cystopteridion s.

Hymenostylium recurvirostre

Barbula crocea

Pinguicula alpina

Carex brachystachys

Tofieldia calyculata iagnostične vrste asociacije)

Pr. Fr.

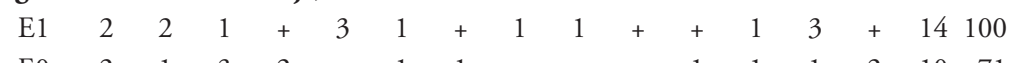

$\mathrm{E} 1 .++\quad+\quad+\quad 1 \quad 1+1 .+1071$

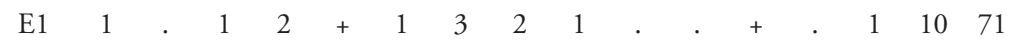

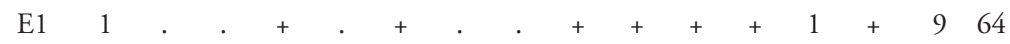

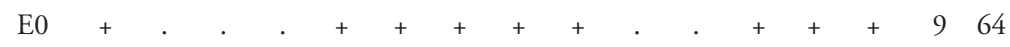

$\begin{array}{llllllllllllllllllll}\mathrm{E} 0 & 2 & 1 & 3 & 2 & . & 1 & 1 & . & . & . & 1 & 1 & 1 & 2 & 10 & 71\end{array}$ 
$\begin{array}{lllllllllllllllllll}\text { Number of relevé (Zaporedna štev. popisa) } & 1 & 2 & 3 & 4 & 5 & 6 & 7 & 8 & 9 & 10 & 11 & 12 & 13 & 14 & \text { Pr. } & \text { Fr. }\end{array}$

Orthothecium rufescens $\quad$ E0

Aster bellidiastrum

E1

Pellia endivifolia

Jungermannia atrovirens

Valeriana tripteris

Fissidens dubius

Paederota lutea

Valeriana saxatilis

Asplenium viride

Viola biflora

PcSp Physoplexido comosae-Saxifragion petraeae

Campanula carnica

E1

Hieracium pospichalii

PC Potentilletalia caulescentis

Potentilla caulescens

$$
\text { E1 }
$$

Primula auricula

Rhamnus pumilus

AT Asplenietea trichomanis

Asplenium ruta-muraria

$$
\text { E1 }
$$

TR Thlaspietea rotundifolii

Hieracium porrifolium

Petasites paradoxus

Hieracium bifidum

Adenostyles glabra

Leontodon hispidus subsp. hyoseroides

Trisetum argenteum

Achnatherum calamagrostis

CD Caricetalia davallianae

Carex flava agg.

Epilobium alsinifolium

Pinguicula vulgaris

\section{Montio-Cardaminetea}

Gymnostomum aeruginosum

Campylium stellatum

Conocephalum conicum

Bryum pseudotriquetrum

Oxyrrhynchium schleicheri

Cololejeunea calcarea

\begin{tabular}{|c|c|c|c|c|c|c|c|c|c|c|c|c|c|c|c|}
\hline E1 & . & . & . & + & + & + & + & 1 & + & & . & . & . & 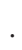 & 6 \\
\hline E1 & + & . & + & . & + & . & + & & . & & . & . & • & & 4 \\
\hline E1 & . & . & . & + & . & + & + & . & . & & . & . & 1 & r & 4 \\
\hline E1 & . & . & . & . & . & . & . & . & . & & + & $\cdot$ & $\mathrm{r}$ & 1 & 3 \\
\hline E1 & + & + & . & . & . & . & . & . & . & & . & . & . & . & 2 \\
\hline E1 & . & . & 1 & . & . & . & . & . & . & & . & + & . & . & 2 \\
\hline E1 & . & . & . & . & + & . & . & . & . & . & . & . & . & . & 1 \\
\hline
\end{tabular}

Barbilophozia sp.

ES Elyno-Seslerietea

Sesleria caerulea

Carex mucronata

Carex ornithopodoides

Carex ferruginea
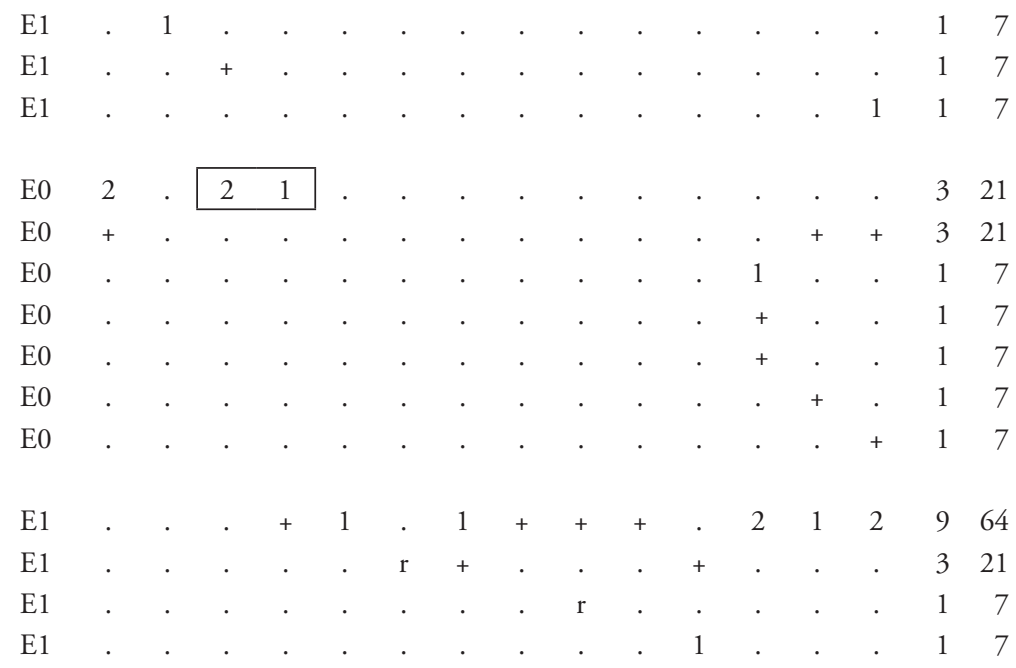

FB Festuco-Brometea

Buphthalmum salicifolium

E1

MA Molinio-Arrhenatheretea

Caltha palustris

E1

$2+$

Angelica sylvestris 


\section{$\begin{array}{llllllllllllllllll}\text { Number of relevé (Zaporedna štev. popisa) } & 1 & 2 & 3 & 4 & 5 & 6 & 7 & 8 & 9 & 10 & 11 & 12 & 13 & 14 & \text { Pr. } & \text { Fr. }\end{array}$}

MuA Mulgedio-Aconitetea

Chaerophyllum hirsutum

E1

Petasites hybridus

E1

EA Epilobietea angustifolii

Eupatorium cannabinum

Tussilago farfara

EP Erico-Pinetea

Erica carnea

Leontodon incanus

Peucedanum austriacum

Carex ornithopoda

\begin{tabular}{|c|c|c|c|c|c|c|c|c|c|c|c|c|c|c|c|}
\hline E1 & . & . & . & 1 & + & + & r & . & . & . & . & . & . & . & 3 \\
\hline E1 & + & . & . & . & . & . & - & . & . & • & . & . & . & . & 1 \\
\hline E1 & . & . & . & . & + & + & 1 & + & + & . & 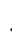 & . & . & . & 5 \\
\hline E1 & . & . & . & + & + & . & . & - & + & . & . & . & . & . & 3 \\
\hline E1 & . & . & . & . & . & + & . & . & + & . & . & . & . & . & 2 \\
\hline E1 & . & . & . & . & . & . & . & . & + & • & . & . & . & . & 1 \\
\hline E1 & . & • & . & + & - & . & . & & . & + & & + & + & 1 & 5 \\
\hline E1 & . & . & . & . & . & . & . & . & . & . & . & . & + & + & 2 \\
\hline
\end{tabular}

AF Aremonio-Fagion

Veronica urticifolia

Saxifraga cuneifolia

E1

Lamium orvala

Primula vulgaris

TA Tilio-Acerion

Acer pseudoplatanus

Tilia platyphyllos

Aruncus dioicus

FS Fagetalia sylvaticae

Petasites albus

Galium laevigatum

Brachypodium sylvaticum

Salvia glutinosa

QP Quercetalia pubescenti-petraeae

Carex flacca

Ostrya carpinifolia

Fraxinus ornus

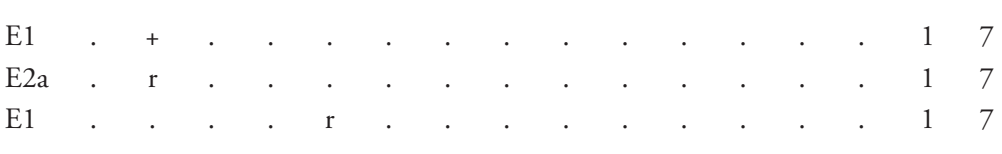

$\mathrm{E} 1$. . . + . . . . . . . . . 2 . 214

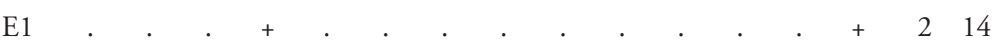

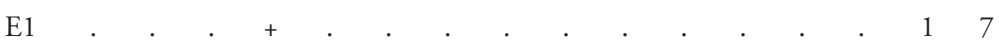

E1 . . . . . . . . . . +17

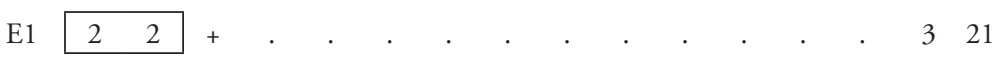

$\mathrm{E} 2 \mathrm{a} .+.+.+.+.+2 .+321$

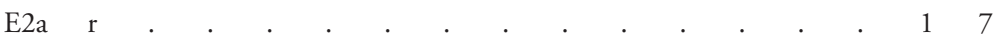

QF Querco-Fagetea

Hedera helix

$\mathrm{E} 1$. . . + . . . . . . . . . . . 17

Carex digitata

E1

SP Salicetea purpureae

Salix purpurea

$\mathrm{E} 2 \mathrm{a} . \mathrm{r} . \quad . \quad . \quad . \quad . \quad . \quad . \quad . \quad 17$

ML Mosses and lichens (Mahovi in lišaji)

Tortella tortuosa

$\mathrm{E} 0$. . . $+.4+. .4429$

Brachythecium rutabulum

Rhynchostegium riparioides

E0

E0

Ctenidium molluscum

E0

Encalypta streptocarpa

E0

\section{Legend / Legenda}

C Chert-roženec

D Dolomite-dolomit

L Limestone - apnenec

M Marlstone - laporovec

Li Lithosol - kamnišče

Pr. Presence (number of relevés in which the species is presented) - število popisov, v katerih se pojavlja vrsta

Fr. Frequency in $\%$ - frekvenca v \% 
Table 4 (Tabela 4): Primuletum carniolicae Accetto 2008 violetosum biflorae.

\section{Number of relevé (Zaporedna številka popisa)}

Database number of relevé (Delovna štev. popisa)

Elevation in $\mathrm{m}$ (Nadmorska višina $\mathrm{v} \mathrm{m}$ )

Aspect (Lega)

Slope in degrees (Nagib v stopinjah)

Parent material (Matična podlaga)

Soil (Tla)

Stoniness in \% (Kamnitost v \%)

Cover of herb layer in \%

$$
\text { (Zastiranje zeliščne plasti v \%) }
$$

Cover of moss layer in \%

$$
\text { (Zastiranje mahovne plasti v \%) }
$$

Number of species (Število vrst)

Relevé area (Velikost popisne ploskve)

Date of taking relevé (Datum popisa)

Locality (Nahajališče)

Quadrant (Kvadrant)

Coordinate GK Y (D-48)

Coordinate GK X (D-48)

\section{$\begin{array}{lllllllllllllllll}1 & 2 & 3 & 4 & 5 & 6 & 7 & 8 & 9 & 10 & 11 & 12 & 13 & 14 & 15 & \text { Pr. } & \text { Fr. }\end{array}$}

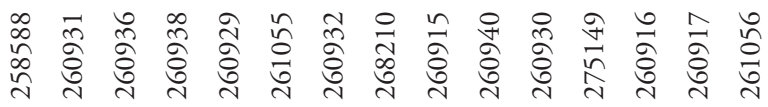

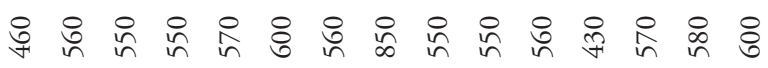

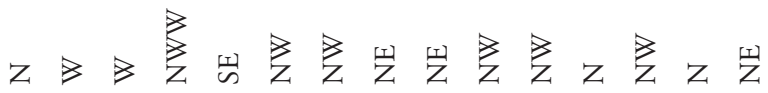
$\begin{array}{lllllllllllllll}90 & 95 & 85 & 90 & 90 & 70 & 95 & 90 & 80 & 80 & 80 & 80 & 80 & 90 & 95\end{array}$ $\begin{array}{lllllllllllllll}\mathrm{D} & \mathrm{D} & \mathrm{D} & \mathrm{D} & \mathrm{D} & \mathrm{D} & \mathrm{D} & \mathrm{D} & \mathrm{D} & \mathrm{D} & \mathrm{D} & \mathrm{D} & \mathrm{D} & \mathrm{D} & \mathrm{D}\end{array}$

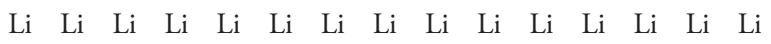
100100100100100100100100100100100100100100100

$\begin{array}{llllllllllllllll}\text { E1 } & 20 & 15 & 30 & 20 & 20 & 30 & 20 & 20 & 40 & 60 & 20 & 20 & 30 & 50 & 30\end{array}$

$\begin{array}{llllllllllllllll}\text { E0 } & 10 & 20 & 20 & 10 & 30 & 30 & 20 & 20 & 60 & 80 & 20 & 40 & 20 & 30 & 10\end{array}$

$\begin{array}{lllllllllllllll}18 & 15 & 28 & 28 & 23 & 26 & 14 & 14 & 17 & 26 & 20 & 17 & 22 & 22 & 7\end{array}$

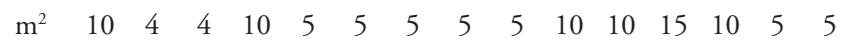

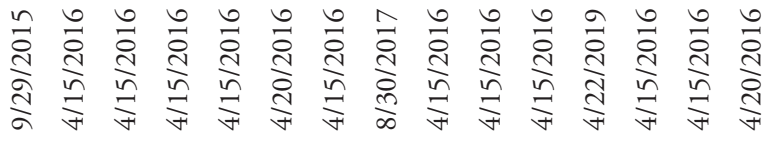

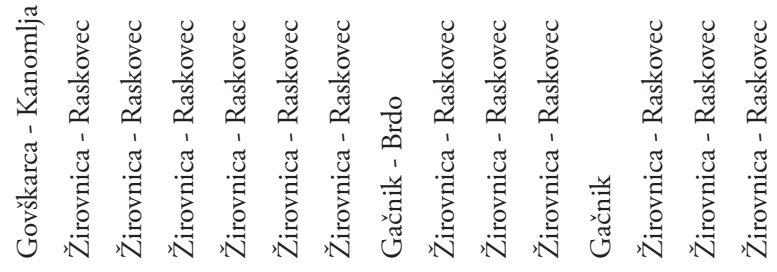

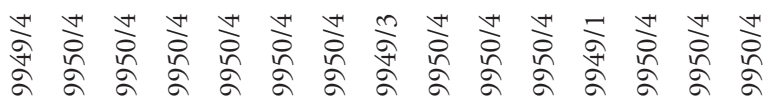

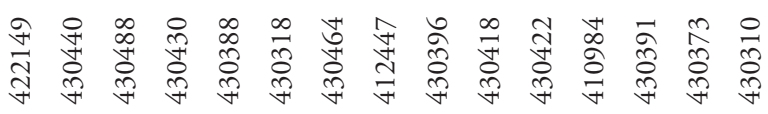
$\mathrm{m}$

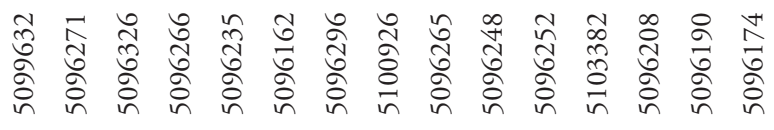

Diagnostic species of the association (Diagnostične vrste asociacije)

AP Orthothecium rufescens

$\begin{array}{llllllllllllllllll}\text { E1 } & 2 & 1 & 2 & 2 & 2 & 1 & 2 & 2 & 1 & 1 & 2 & 1 & 2 & 2 & 1 & 15 & 100\end{array}$

AP Valeriana tripteris

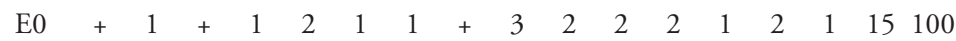

Differential species of the subassociation (Razlikovalnice asociacije)

AP Viola biflora

VP Veronica urticifolia

AP Pinguicula alpina

Cystopteridion s. lat. (Astrantio-Paederotion luteae nom. prov.)

Aster bellidiastrum

Asplenium viride

Cystopteris fragilis

Carex brachystachys

Paederota lutea

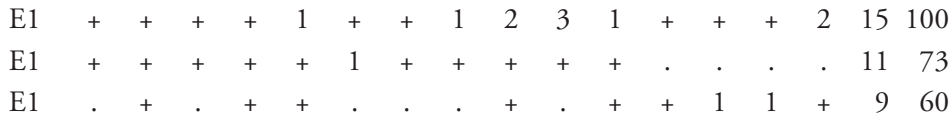

$\mathrm{E} 11++111+.1+11493$

$\mathrm{E} 1+1++11 .+2 .+1.960$

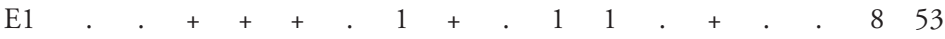

$\mathrm{E} 1+\cdot+1 \cdot+\cdot+\cdot \cdot 1 \cdot++.74$

E1 1 . . . . . . + . . 1 . . 320 
Number of relevé (Zaporedna številka popisa)

Hymenostylium recurvirostre

Tofieldia calyculata

Palustriella commutata

Moehringia muscosa

Astrantia carniolica

Fissidens dubius

Valeriana saxatilis

PcSp Physoplexido comosae-Saxifragion petraeae

Campanula cespitosa

Phyteuma scheuchzeri subsp. columnae

AT Asplenietea trichomanis

Asplenium ruta-muraria

Asplenium trichomanes

TR Thlapietea rotundifolii

Hieracium bifidum

Gymnocarpium robertianum

MC Montio-Cardaminetea

Conocephalum conicum

Oxyrrhynchium hians

Campylium stellatum

Oxyrrhynchium schleicheri

Cirriphyllum cirrhosum

Distichium capillaceum

ES Elyno-Seslerietea

Sesleria caerulea

Carex mucronata

FB Festuco-Brometea

Buphthalmum salicifolium

BA Betulo-Alnetea

Salix appendiculata

MuA Mulgedio-Aconitetea

Petasites hybridus

EP Erico-Pinetea

Calamagrostis varia

Rhododendron hirsutum

Rubus saxatilis

Molinia arundinacea

Erica carnea

VP Vaccinio-Piceetea

Veronica urticifolia

Homogyne sylvestris

Oxalis acetosella

Clematis alpina

Rosa pendulina

Aposeris foetida

AF Aremonio-Fagion

Scopolia carniolica

Cyclamen purpurascens

Helleborus niger
E0

E1

E0

E1

E1

E0

E1

E1

E1

$\mathrm{E} 1+\mathrm{r}++++++.+\mathrm{r}+.1173$

$\mathrm{E} 1+++1+.11 .+2.960$

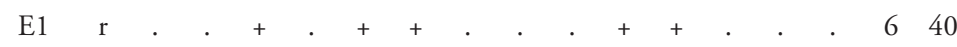

E1

$\mathrm{E} 011+++1 .+2212 .+1280$

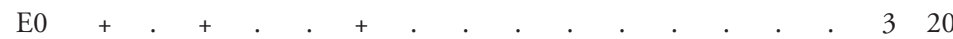

$\mathrm{E} 0$. . . . + . . . . . . . . 213

$\mathrm{E} 0$. . ......+++2.213

$\mathrm{E} 0$. . . . $+. . .5 . . .517$

E0 . . . . . . . . . . . . . 17

E1

E1

E1

$\mathrm{E} 1++++.+2 .+++++2.1067$

$\mathrm{E} 1 . .5 . \quad . \quad . \quad . \quad . \quad+1.213$

E1

E1

E1

$\mathrm{E} 1+++++1++{ }_{+}+{ }_{+} .+1173$

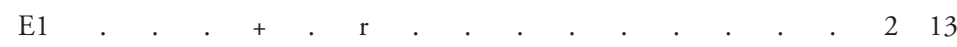

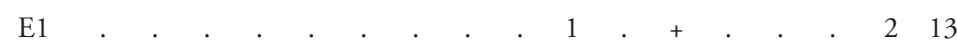

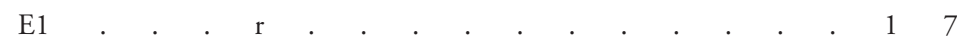

$\mathrm{E} 1 . . \quad . \quad \mathrm{r} . \quad . \quad . \quad . \quad . \quad . \quad . \quad . \quad 17$

$\mathrm{E} 1 . . .5 . \quad . \quad . \quad . \quad . \quad . \quad .117$

$\mathrm{E} 1 . \quad . \quad \mathrm{r} . \quad . \quad . \quad . \quad . \quad . \quad . \quad \mathrm{r} \quad . \quad 213$

$\mathrm{E} 1 . . \quad \mathrm{r} . \quad . \quad . \quad . \quad+. \quad .2213$

$\mathrm{E} 1 . . \quad . \quad \mathrm{r} . \quad . \quad . \quad . \quad . \quad . \quad . \quad 17$ 
Number of relevé (Zaporedna številka popisa)

Cardamine enneaphyllos

Euphorbia carniolica

Cardamine trifolia

TA Tilio-Acerion

Aruncus dioicus

Thalictrum aquilegiifolium

Polystichum aculeatum

Ulmus glabra

Acer pseudoplatanus

Phyllitis scolopendrium

FS Fagetalia sylvaticae

Galeobdolon flavidum

Cardamine pentaphyllos

Campanula trachelium

Lathyrus vernus

Galium laevigatum

Salvia glutinosa

Daphne mezereum

Mercurialis perennis

Mycelis muralis

Fagus sylvatica

Prenanthes purpurea

QF Querco-Fagetea

Carex digitata

Hepatica nobilis

Taxus baccata

ML Mosses and lichens (Mahovi in lišaji)

Neckera crispa

Ctenidium molluscum

Mnium thomsonii

Tortella tortuosa

Pedinophyllum interruptum

Mnium marginatum

Brachythecium starkei

Plagiomnium rostratum

Marchantia polymorpha
E1

E1

E1

E1

E1

E1

E2a

E1

E1

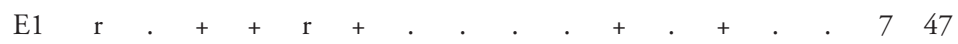

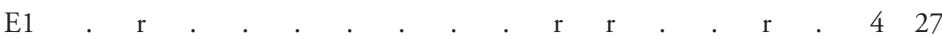

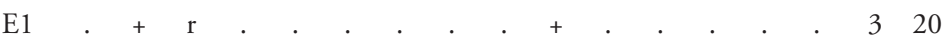

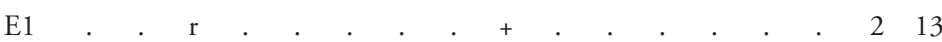

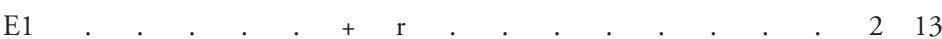

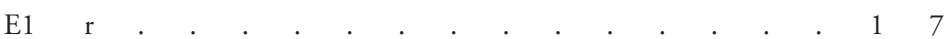

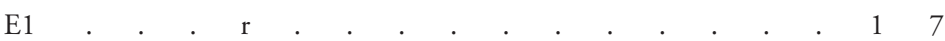

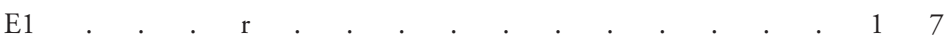

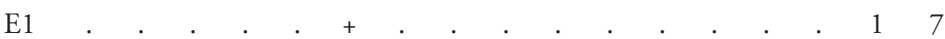

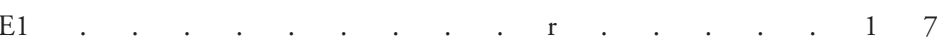

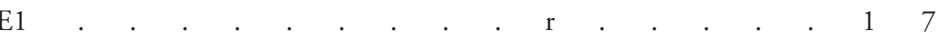

$\mathrm{E} 1$. . $\mathrm{r} \cdot++.+\mathrm{r}+\mathrm{r}+.960$

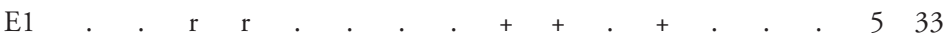

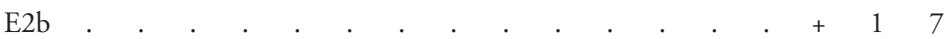

$\mathrm{E} 0 . .++21+. .5+. .640$

$\mathrm{E} 0$. . . . . ..+211.533

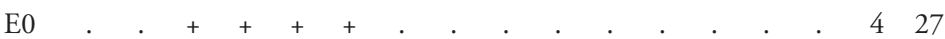

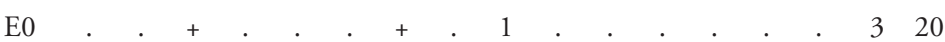

$\mathrm{E} 0$. . . $1+. . .51 .320$

$\mathrm{E} 0$. . . + . . . . . . . 320

$\mathrm{E} 0$. . . . . . . . . . . 17

$\mathrm{E} 0$. . . . $+. . . \quad . \quad . \quad . \quad . \quad . \quad 17$

\section{Legend / Legenda}

D Dolomite - dolomit

Li Lithosol - kamnišče

Pr. Presence (number of relevés in which the species is presented) - število popisov, v katerih se pojavlja vrsta

Fr. Frequency in $\%$ - frekvenca $v \%$ 
Table 5 (Tabela 5): Phyteumato columnae-Primuletum carniolicae. Relevé number 1-37.

\begin{tabular}{|c|c|c|c|c|c|c|c|c|c|c|c|c|c|}
\hline Number of relevé (Zaporedna številka popisa) & & 1 & 2 & 3 & 4 & 5 & 6 & 7 & 8 & 9 & 10 & 11 & 12 \\
\hline Database number of relevé (Delovna številka popisa) & & 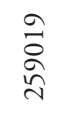 & 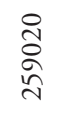 & $\begin{array}{l}\infty \\
\stackrel{\sim}{\sim} \\
\stackrel{\sim}{2}\end{array}$ & $\begin{array}{l}\stackrel{+}{\infty} \\
\stackrel{\infty}{\sim} \\
\vec{v}\end{array}$ & 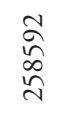 & 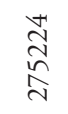 & 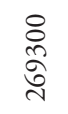 & $\begin{array}{l}n \\
\stackrel{D}{ } \\
\stackrel{\infty}{v}\end{array}$ & $\begin{array}{l}\stackrel{0}{0} \\
\stackrel{\infty}{\curvearrowright} \\
\stackrel{\sim}{N}\end{array}$ & $\begin{array}{l}\text { त్ } \\
\text { त } \\
\text { }\end{array}$ & $\frac{\stackrel{+}{n}}{\stackrel{0}{0}}$ & $\begin{array}{l}\stackrel{\widehat{\widehat{े}}}{\text { ठे }} \\
\text { }\end{array}$ \\
\hline Elevation in $\mathrm{m}$ (Nadmorska višina $\mathrm{v} \mathrm{m}$ ) & & $\hat{\kappa}$ & $\hat{\kappa}$ & $\underset{f}{i}$ & i & $\begin{array}{l}n \\
\stackrel{\infty}{+} \\
+\end{array}$ & $\underset{\sim}{~}$ & $\stackrel{n}{\forall}$ & 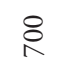 & : & $\stackrel{\sim}{\sim}$ & $\stackrel{\circ}{\sim}$ & $\stackrel{n}{\forall}$ \\
\hline Aspect (Lega) & & $\mathrm{NE}$ & $\mathrm{NE}$ & $\mathrm{NE}$ & SW & $S$ & E & NW & $\mathrm{N}$ & $\mathrm{N}$ & NW & $\mathrm{N}$ & $\mathrm{NE}$ \\
\hline Slope in degrees (Nagib v stopinjah) & & 80 & 90 & 90 & 90 & 80 & 95 & 70 & 90 & 80 & 90 & 90 & 90 \\
\hline Parent material (Matična podlaga) & & $\mathrm{D}$ & $\mathrm{D}$ & $\mathrm{D}$ & $\mathrm{D}$ & $\mathrm{D}$ & $\mathrm{D}$ & $\mathrm{D}$ & $\mathrm{D}$ & $\mathrm{D}$ & $\mathrm{D}$ & $\mathrm{D}$ & $\mathrm{D}$ \\
\hline Soil (Tla) & & $\mathrm{Li}$ & $\mathrm{Li}$ & $\mathrm{Li}$ & $\mathrm{Li}$ & $\mathrm{Li}$ & $\mathrm{Li}$ & $\mathrm{Li}$ & $\mathrm{Li}$ & $\mathrm{Li}$ & $\mathrm{Li}$ & $\mathrm{Li}$ & $\mathrm{Li}$ \\
\hline Stoniness in \% (Kamnitost v \%) & & 100 & 100 & 100 & 100 & 100 & 100 & 100 & 100 & 100 & 100 & 100 & 100 \\
\hline Cover of herb layer in \% (Zastiranje zeliščne plasti v \%) & E1 & 30 & 20 & 30 & 30 & 40 & 40 & 35 & 20 & 30 & 20 & 25 & 30 \\
\hline Cover of moss layer in \% (Zastiranje mahovne plasti v \%) & E0 & 30 & 20 & 30 & 10 & 20 & 10 & 50 & 10 & 5 & 10 & 20 & 20 \\
\hline Number of species (Število vrst) & & 24 & 24 & 16 & 17 & 13 & 10 & 20 & 27 & 20 & 11 & 10 & 14 \\
\hline Relevé area (Velikost popisne ploskve) & $\mathrm{m}^{2}$ & 10 & 10 & 10 & 10 & 3 & 10 & 10 & 15 & 10 & 5 & 10 & 10 \\
\hline Date of taking relevé (Datum popisa) & & 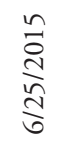 & 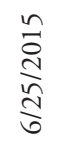 & 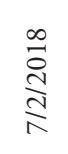 & 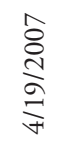 & 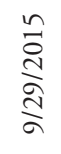 & 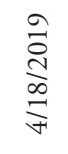 & 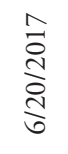 & 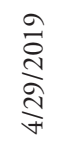 & 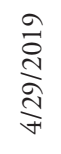 & 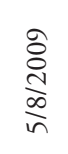 & $\begin{array}{l}\frac{N}{\vec{N}} \\
\frac{a}{a}\end{array}$ & 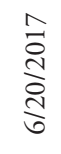 \\
\hline Locality (Nahajališce) & & 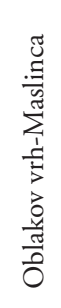 & 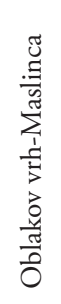 & $\begin{array}{l}\text { 莺 } \\
\text { 岛 }\end{array}$ & 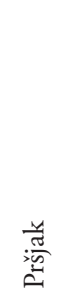 & 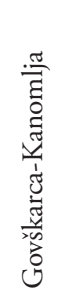 & 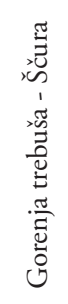 & 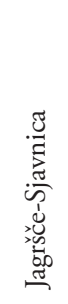 & 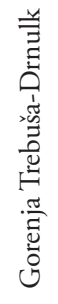 & 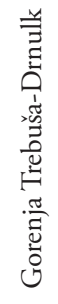 & 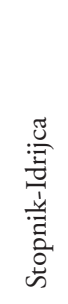 & 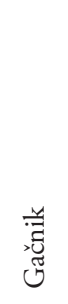 & 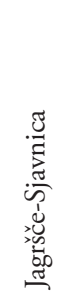 \\
\hline Quadrant (Kvadrant) & & $\begin{array}{l}\vec{\sigma} \\
\stackrel{\sigma}{\alpha}\end{array}$ & $\begin{array}{l}\bar{a} \\
\stackrel{+}{a}\end{array}$ & $\begin{array}{l}\vec{a} \\
\stackrel{+}{a} \\
\text { a }\end{array}$ & $\begin{array}{l}\bar{a} \\
\stackrel{+}{a}\end{array}$ & $\begin{array}{l}\frac{1}{a} \\
\stackrel{+}{\sigma}\end{array}$ & $\begin{array}{l}\stackrel{v}{\infty} \\
\stackrel{+}{\sigma} \\
\stackrel{\sigma}{ }\end{array}$ & $\begin{array}{l}\frac{N}{\sigma} \\
\sigma \\
\sigma\end{array}$ & $\begin{array}{l}\frac{N}{\infty} \\
\stackrel{+}{\alpha} \\
\stackrel{\sigma}{\alpha}\end{array}$ & $\begin{array}{l}\frac{v}{\infty} \\
\stackrel{+}{\sigma} \\
\sigma\end{array}$ & 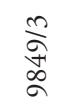 & $\begin{array}{l}\bar{a} \\
\stackrel{+}{a}\end{array}$ & $\begin{array}{l}\frac{N}{\alpha} \\
\sigma \\
\sigma\end{array}$ \\
\hline Coordinate GK Y (D-48) & $\mathrm{m}$ & 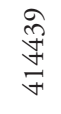 & 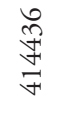 & 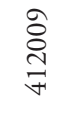 & 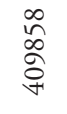 & 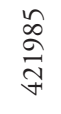 & $\begin{array}{l}\text { ते } \\
\text { ○े } \\
\text { 하 }\end{array}$ & $\begin{array}{l}\text { o } \\
\stackrel{0}{\infty} \\
\forall \\
\forall\end{array}$ & $\begin{array}{l}\vec{\sigma}+\overrightarrow{+} \\
\text { o } \\
\stackrel{+}{+}\end{array}$ & 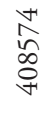 & 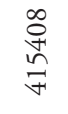 & 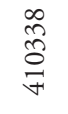 & $\begin{array}{l}\widehat{\widehat{े}} \\
\infty \\
\vec{\forall}\end{array}$ \\
\hline Coordinate GK X (D-48) & $\mathrm{m}$ & $\begin{array}{l}\stackrel{\infty}{\Omega} \\
\stackrel{0}{n} \\
n\end{array}$ & $\begin{array}{l}\stackrel{\partial}{0} \\
\stackrel{0}{\circ} \\
\vec{n}\end{array}$ & $\begin{array}{l}\grave{\curvearrowright} \\
\overrightarrow{\vec{\sigma}} \\
\vec{n}\end{array}$ & 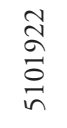 & $\begin{array}{l}\hat{े} \\
\stackrel{\circ}{\circ} \\
\stackrel{\circ}{n}\end{array}$ & $\begin{array}{l}\hat{f} \\
\text { } \\
\text { है } \\
\text { n }\end{array}$ & 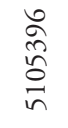 & 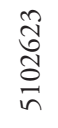 & $\begin{array}{l}\vec{\infty} \\
\stackrel{0}{0} \\
\widetilde{\sigma} \\
\sim\end{array}$ & $\begin{array}{l}0 \\
\infty \\
0 \\
\stackrel{0}{0} \\
\sim\end{array}$ & $\begin{array}{l}\stackrel{0}{\vec{\sigma}} \\
\vec{\sigma} \\
\vec{n}\end{array}$ & $\begin{array}{l}\text { ते } \\
\text { है } \\
\text { है }\end{array}$ \\
\hline
\end{tabular}

Diagnostic species of the association (Diagnostične vrste asociacije)

AP Primula carniolica

PcSp Phyteuma scheuchzeri subsp. columnae

AP Paederota lutea

AP Orthothecium rufescens

AP Valeriana tripteris

AP Carex brachystachys

AP Cystopteridion s. lat. (Astrantio-Paederotion luteae nom. prov.)

Aster bellidiastrum

Asplenium viride

Fissidens dubius

Valeriana saxatilis

Tofieldia calyculata

Viola biflora

Barbula crocea

Cystopteris fragilis

Preissia quadrata

$\begin{array}{lllllllllllll}\text { E1 } & \mathrm{r} & 1 & + & 1 & 3 & 3 & 2 & 2 & 2 & + & + & 1 \\ \text { E1 } & + & + & 1 & + & + & + & + & + & \mathrm{r} & + & + & 1 \\ \text { E1 } & 1 & 2 & 2 & 2 & 1 & 1 & 2 & 2 & 2 & + & 1 & 2 \\ \text { E0 } & + & + & + & + & 2 & 1 & 1 & + & 1 & + & 1 & 1 \\ \text { E1 } & 1 & + & 1 & . & . & . & 1 & \text { r } & . & . & . & . \\ \text { E1 } & + & \mathrm{r} & 2 & + & 1 & 1 & . & . & + & . & . & +\end{array}$




\section{$\begin{array}{lllllllllllllllllllllllll}13 & 14 & 15 & 16 & 17 & 18 & 19 & 20 & 21 & 22 & 23 & 24 & 25 & 26 & 27 & 28 & 29 & 30 & 31 & 32 & 33 & 34 & 35 & 36 & 37\end{array}$}

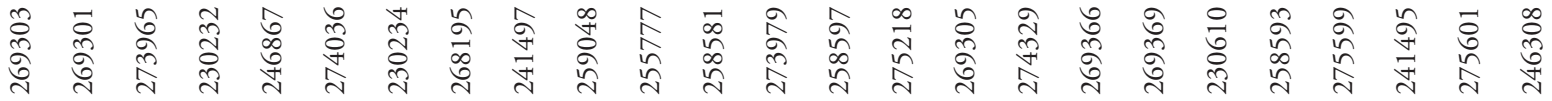

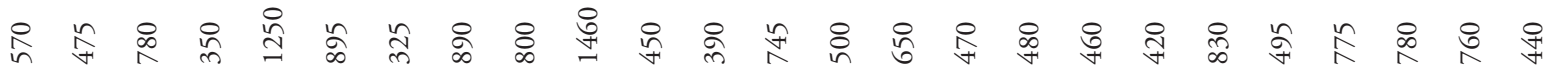

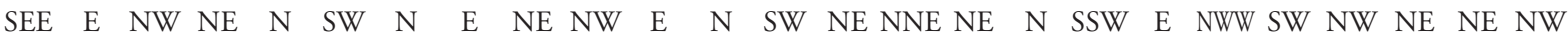
$\begin{array}{lllllllllllllllllllllllll}90 & 80 & 85 & 90 & 80 & 90 & 90 & 85 & 80 & 80 & 80 & 90 & 90 & 90 & 80 & 80 & 85 & 70 & 80 & 95 & 90 & 85 & 80 & 85 & 90\end{array}$ $\begin{array}{llllllllllllllllllllllllllllll}\mathrm{D} & \mathrm{D} & \mathrm{D} & \mathrm{D} & \mathrm{D} & \mathrm{D} & \mathrm{D} & \mathrm{D} & \mathrm{D} & \mathrm{L} & \mathrm{D} & \mathrm{D} & \mathrm{D} & \mathrm{D} & \mathrm{D} & \mathrm{D} & \mathrm{D} & \mathrm{D} & \mathrm{D} & \mathrm{D} & \mathrm{D} & \mathrm{D} & \mathrm{D} & \mathrm{D} & \mathrm{D}\end{array}$

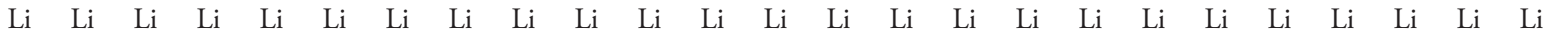
$\begin{array}{lllllllllllllllllllllllllll}100 & 100 & 100 & 100 & 100 & 100 & 100 & 100 & 100 & 100 & 100 & 100 & 100 & 100 & 100 & 100 & 100 & 100 & 100 & 100 & 100 & 100 & 100 & 100 & 100\end{array}$ $\begin{array}{lllllllllllllllllllllllll}25 & 30 & 15 & 30 & 35 & 20 & 20 & 30 & 30 & 30 & 30 & 20 & 30 & 30 & 30 & 40 & 30 & 30 & 20 & 30 & 30 & 20 & 20 & 25 & 20\end{array}$ $\begin{array}{llllllllllllllllllllllllll}10 & 30 & 20 & 10 & 20 & 30 & 30 & 20 & 10 & 20 & 20 & 10 & 10 & 20 & 30 & 40 & 20 & 20 & 20 & 5 & 10 & 10 & 5 & 10 & 20\end{array}$ $\begin{array}{llllllllllllllllllllllllll}13 & 17 & 15 & 29 & 32 & 14 & 18 & 15 & 24 & 15 & 25 & 12 & 10 & 18 & 15 & 26 & 26 & 15 & 15 & 17 & 11 & 14 & 16 & 15 & 20\end{array}$ $\begin{array}{llllllllllllllllllllllllllllll}10 & 10 & 10 & 10 & 10 & 10 & 10 & 5 & 10 & 4 & 5 & 4 & 10 & 4 & 10 & 10 & 10 & 5 & 5 & 10 & 2 & 10 & 10 & 10 & 10\end{array}$

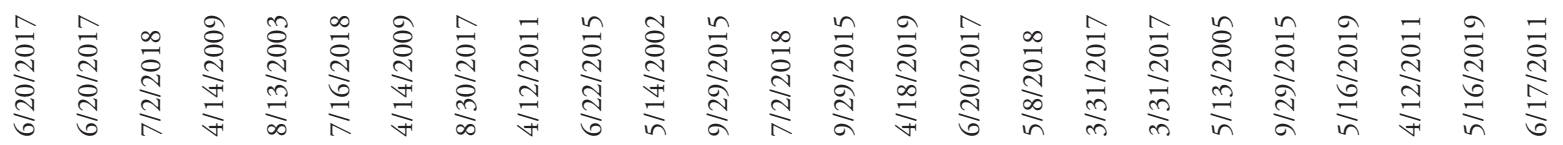

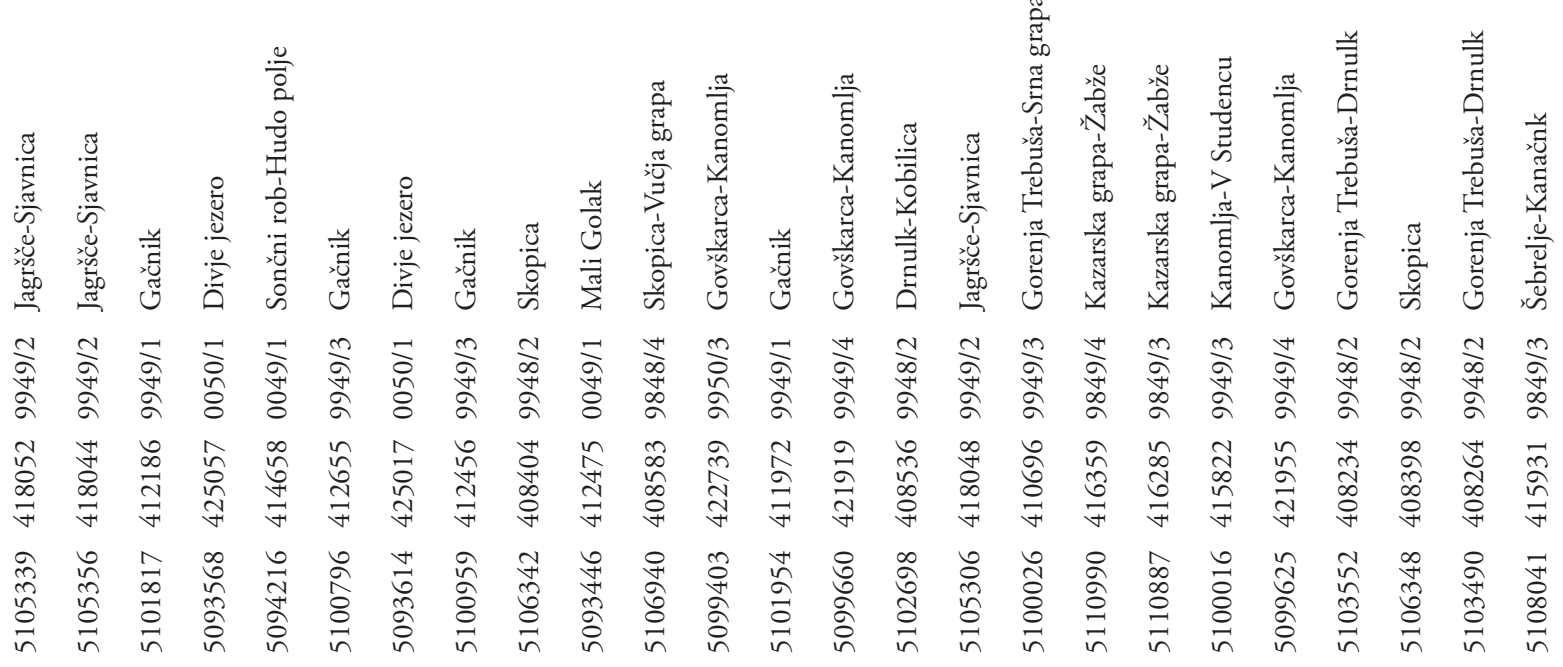


Hymenostylium recurvirostre

Moehringia muscosa

Primula $x$ venusta

Pellia endiviifolia

Selaginella helvetica

Palustriella commutata

Heliosperma veselskyi subsp. veselskyi

PcSp Physoplexido comosae-Saxifragion petraeae

Campanula cespitosa

Athamanta turbith

Hieracium porrifolium

Potentilletalia caulescentis

Potentilla caulescens

Kernera saxatilis

Primula auricula

AT Asplenietea trichomanis

Asplenium ruta-muraria

Asplenium trichomanes

Hieracium glaucum

TR Thlaspietea rotundifolii

Hieracium bifidum

Gymnocarpium robertianum

Adenostyles glabra

Aquilegia iulia

Dryopteris villarii

MC Montio-Cardaminetea

Conocephalum conicum

Distichium capillaceum

Ditrichum flexicaule

Campylium stellatum

Cololejeunea calcarea

S Elyno-Seslerietea

Sesleria caerulea

Carex mucronata

Betonica alopecuros

Carduus crassifolius

Carex ferruginea

Gentiana clusii

Laserpitium peucedanoides

FB Festuco-Brometea

Carex humilis

Buphthalmum salicifolium

Galium lucidum

Brachypodium rupestre

E1

E1

E1

E1

E1

Molinio-Arrbenatheretea

Angelica sylvestris

Caltha palustris

Taraxacum $x$ Ruderalia

BA Betulo-Alnetea

Salix appendiculata

Salix glabra

MuA Mulgedio-Aconitetea

Senecio ovatus

Ranunculus platanifolius

Aconitum degenii subsp. paniculatum

Saxifraga rotundifolia

E1

E1

TG Trifolio-Geranietea

Laserpitium siler

Viola hirta 


\section{Number of relevé (Zaporedna številka popisa)}

Anthericum ramosum

Laserpitium latifolium

Thalictrum minus

EA Epilobietea angustifolii

Rubus idaeus

EP Erico-Pinetea

Calamagrostis varia

Rhododendron hirsutum

Erica carnea

Polygala chamaebuxus

Carex ornithopoda

Rubus saxatilis

Carex alba

Cirsium erisithales

Leontodon incanus

Molinia arundinacea

Amelanchier ovalis

Aster amellus

Rhodothamnus chamaecistus

Aquilegia nigricans

Pinus sylvestris

VP Vaccinio-Pinetea

Veronica urticifolia

Homogyne sylvestris

Solidago virgaurea

Hieracium murorum

Clematis alpina

Gentiana asclepiadea

Oxalis acetosella

Picea abies

Polystichum lonchitis

Aposeris foetida

Rosa pendulina

AF Aremonio-Fagion

Cyclamen purpurascens

Helleborus niger

Hemerocallis lilioasphodelus

Potentilla carniolica

Cardamine trifolia

Primula vulgaris

Anemone trifolia

Euphorbia carniolica

Lamium orvala

Rhamnus fallax

Laserpitium krapfii

Omphalodes verna

Knautia drymeia

TA Tilio-Acerion

Aruncus dioicus

Polystichum aculeatum

Phyllitis scolopendrium

Acer pseudoplatanus

Geranium robertianum

Tephroseris pseudocrispa

Thalictrum aquilegiifolium

Ulmus glabra

Acer platanoides

\section{$\begin{array}{llllllllllll}1 & 2 & 3 & 4 & 5 & 6 & 7 & 8 & 9 & 10 & 11 & 12\end{array}$}

E1

E1

E1

E1

E1

E1

E1

E1

E1

E1

E1

E1

E1

E1

E1

E1

E1

E1

E2a

E1

E1

E1

E1

E1

E1

E1

E1

E1

E1

E1

E1

E1

E1

E1

E1

E1

E1

E1

E1

E1

E1

E1

E1

E1

E1

E1 


\section{Number of relevé (Zaporedna števillka popisa)}

\section{$\begin{array}{llllllllllll}1 & 2 & 3 & 4 & 5 & 6 & 7 & 8 & 9 & 10 & 11 & 12\end{array}$}

FS Fagetalia sylvaticae

Salvia glutinosa

Galeobdolon flavidum

Mercurialis perennis

Mycelis muralis

Galium laevigatum

Fagus sylvatica

Melica nutans

Symphytum tuberosum

Campanula trachelium

Cardamine pentaphyllos

Asarum europaeum subsp. caucasicum

Petasites albus

Sambucus nigra

Lathyrus vernus

E1

E1

E1

E1

E1

E1

E1

E1

E1

E1

E1

E1

E2a

E1

E1

E1

E1

E1

E2a

Euonymus verrucosa

QF Querco-Fagetea

Carex digitata

Hepatica nobilis

Hedera helix

Veratrum nigrum

Acer campestre

Clematis vitalba

Quercus petraea

Cardamine impatiens

Lonicera xylosteum

E1

E1

E1

E1

E1

E1

E1

E1

E1

ML Mosses and lichens (Mahovi in lišaji)

Neckera crispa

Ctenidium molluscum

Tortella tortuosa

Mnium thomsonii

Schistidium apocarpum

Plagiochila porreloides

Encalypta streptocarpa

Leiocolea collaris

Marchantia polymorpha

Bryum capillare

Pedinophyllum interruptum

Mnium stellare

Isothecium alopecuroides

Lejeunea cavifolia

Myurella sibirica

Neckera complanata

Didymodon ferrugineus

Trichostomum brachydontium

Myurella julacea

Hypnum cupressiforme var. resupinatum

Lichenes div.

Lophozia sp.

Dichodontium pellucidum subsp. flavescens

Brachythecium rutabulum

Plagiomnium rostratum

Mnium marginatum

Plagiobryum zierii

\section{(}

(1)

E0

E0

E0

E0

E0

E0

E0

E0

E0

E0

E0

E0

E0

E0

E0

E0

E0

E0

E0

E0

E0

E0

E0

E0

E0

(1)

.

(

.

1

a

.

.

a

.

1

.

.

$\begin{array}{lllllllllllll}\text { E0 } & 1 & 1 & 1 & . & + & . & . & + & . & . & . & . \\ \text { E0 } & 1 & 1 & . & . & . & . & + & + & . & . & . & . \\ \text { E0 } & . & . & 1 & + & 1 & . & 2 & 1 & + & 1 & + & 1\end{array}$ 


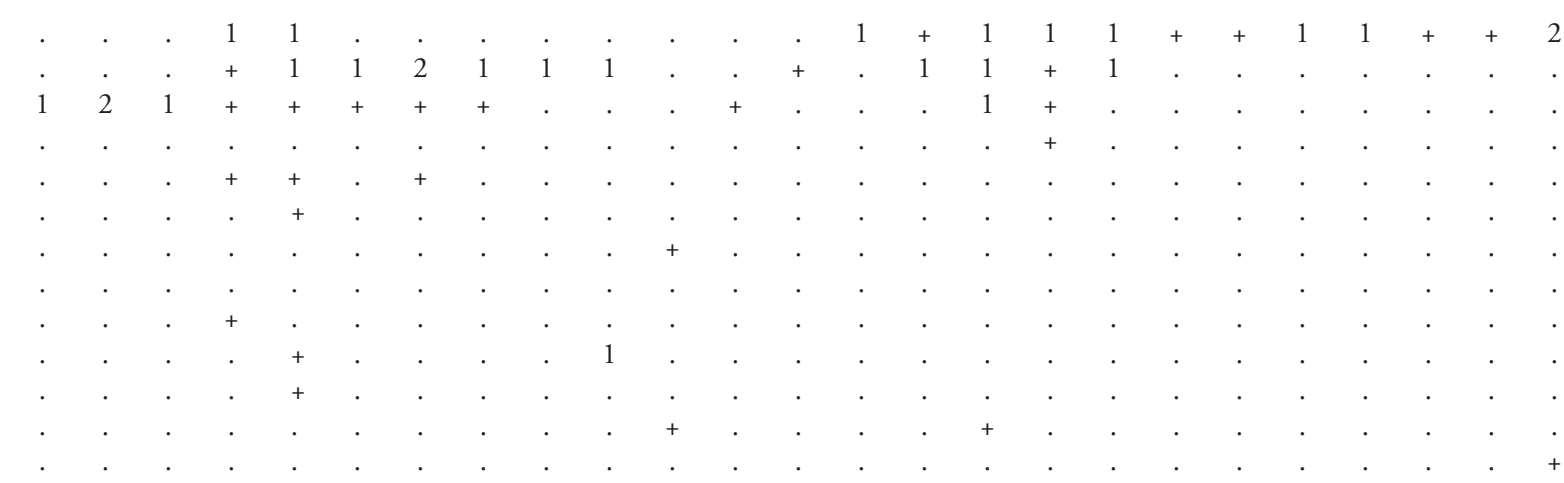


Table 5 (Tabela 5): Phyteumato columnae-Primuletum carniolicae. Relevé number 38-73.

\begin{tabular}{|c|c|c|c|c|c|c|c|c|c|c|c|c|c|}
\hline Number of relevé (Zaporedna številka popisa) & & 38 & 39 & 40 & 41 & 42 & 43 & 44 & 45 & 46 & 47 & 48 & 49 \\
\hline Database number of relevé (Delovna številka popisa) & & $\begin{array}{l}n \\
\stackrel{n}{0} \\
\stackrel{\sim}{n}\end{array}$ & 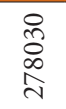 & 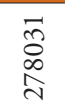 & 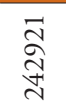 & 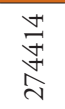 & 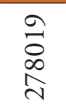 & 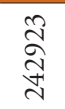 & $\begin{array}{l}\stackrel{\infty}{\hat{n}} \\
\hat{n}\end{array}$ & $\begin{array}{l}\text { } \\
\stackrel{\text { }}{~} \\
\text { సे }\end{array}$ & 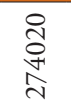 & $\underset{\stackrel{\widetilde{\curvearrowright}}{\sim}}{\stackrel{\sim}{\sim}}$ & $\begin{array}{l}\infty \\
\approx \\
\infty \\
\approx \\
\approx\end{array}$ \\
\hline Elevation in $\mathrm{m}$ (Nadmorska višina $\mathrm{v} \mathrm{m})$ & & 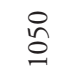 & $\frac{\circ}{a}$ & $\tilde{\approx}$ & $\underset{\infty}{\infty}$ & $\stackrel{n}{\stackrel{n}{n}}$ & $\stackrel{\unrhd}{n}$ & $\underset{\infty}{\stackrel{+}{\infty}}$ & $\stackrel{尺}{\wedge}$ & $\stackrel{n}{\wedge}$ & $\stackrel{\wp}{\infty}$ & $\stackrel{\infty}{\stackrel{一}{ }}$ & 요 \\
\hline Aspect (Lega) & & NW & NW & NW & NE & W & $\mathrm{NE}$ & $\mathrm{N}$ & NNE & S & SSW & $\mathrm{NE}$ & W \\
\hline Slope in degrees (Nagib v stopinjah) & & 90 & 90 & 70 & 90 & 80 & 90 & 90 & 90 & 85 & 90 & 85 & 30 \\
\hline Parent material (Matična podlaga) & & $\mathrm{D}$ & $\mathrm{D}$ & $\mathrm{D}$ & $\mathrm{D}$ & $\mathrm{D}$ & $\mathrm{D}$ & $\mathrm{D}$ & $\mathrm{D}$ & $\mathrm{D}$ & $\mathrm{D}$ & $\mathrm{D}$ & $\mathrm{D}$ \\
\hline Soil (Tla) & & $\mathrm{Li}$ & $\mathrm{Li}$ & $\mathrm{Li}$ & $\mathrm{Li}$ & $\mathrm{Li}$ & $\mathrm{Li}$ & $\mathrm{Li}$ & $\mathrm{Li}$ & $\mathrm{Li}$ & $\mathrm{Li}$ & $\mathrm{Li}$ & $\mathrm{Li}$ \\
\hline Stoniness in \% (Kamnitost v \%) & & 100 & 100 & 100 & 100 & 100 & 100 & 100 & 100 & 100 & 100 & 100 & 100 \\
\hline Cover of herb layer in \% (Zastiranje zeliščne plasti v \%) & E1 & 20 & 20 & 20 & 20 & 30 & 25 & 15 & 20 & 20 & 20 & 30 & 40 \\
\hline Cover of moss layer in \% (Zastiranje mahovne plasti v \%) & E0 & 10 & 10 & 30 & 20 & 40 & 10 & 20 & 20 & 20 & 10 & 20 & 40 \\
\hline Number of species (Število vrst) & & 15 & 18 & 14 & 14 & 20 & 16 & 11 & 14 & 11 & 11 & 10 & 18 \\
\hline Relevé area (Velikost popisne ploskve) & $\mathrm{m}^{2}$ & 10 & 20 & 10 & 10 & 10 & 15 & 10 & 5 & 10 & 10 & 10 & 2 \\
\hline Date of taking relevé (Datum popisa) & & 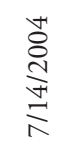 & 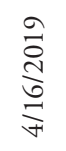 & 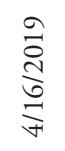 & 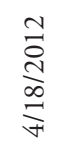 & 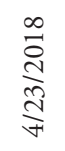 & 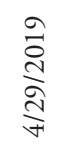 & 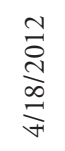 & 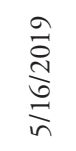 & 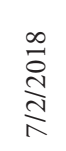 & 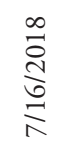 & 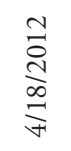 & 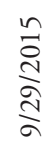 \\
\hline Locality (Nahajališče) & & 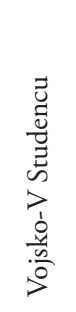 & 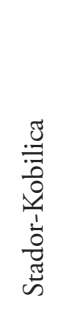 & 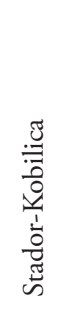 & 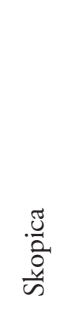 & 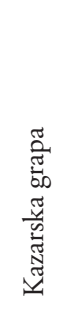 & 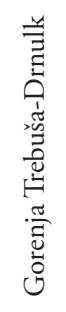 & 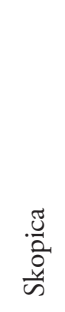 & 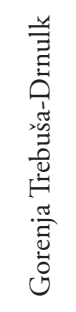 & 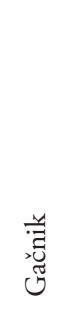 & 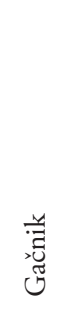 & 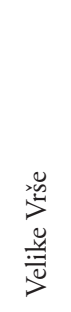 & (2) \\
\hline Quadrant (Kvadrant) & & 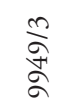 & $\begin{array}{l}\stackrel{v}{\infty} \\
\stackrel{\infty}{+} \\
\stackrel{\sigma}{\sigma}\end{array}$ & $\begin{array}{l}\stackrel{v}{\infty} \\
\stackrel{\infty}{+} \\
\stackrel{\sigma}{\sigma}\end{array}$ & $\begin{array}{l}\stackrel{v}{\infty} \\
\stackrel{\infty}{+} \\
\sigma\end{array}$ & 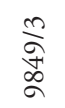 & 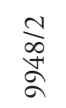 & $\begin{array}{l}\stackrel{N}{\infty} \\
\stackrel{\infty}{+} \\
\sigma\end{array}$ & $\begin{array}{l}\stackrel{v}{\infty} \\
\stackrel{\infty}{+} \\
\sigma\end{array}$ & 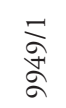 & 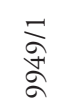 & $\begin{array}{l}\stackrel{N}{\infty} \\
\stackrel{\infty}{+} \\
\stackrel{\sigma}{\sigma}\end{array}$ & \\
\hline Coordinate GK Y (D-48) & $\mathrm{m}$ & 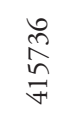 & $\begin{array}{l}\stackrel{\infty}{+} \\
\stackrel{5}{\circ} \\
\stackrel{+}{+}\end{array}$ & 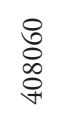 & 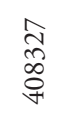 & 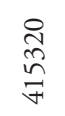 & 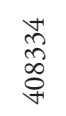 & 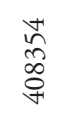 & 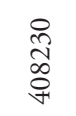 & \begin{tabular}{l}
$\triangleq$ \\
$\underset{\exists}{\Xi}$ \\
\multirow{F}{*}{}
\end{tabular} & $\begin{array}{l}\stackrel{n}{\beth} \\
\underset{\forall}{\approx}\end{array}$ & 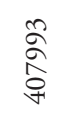 & \\
\hline Coordinate GK X (D-48) & $\mathrm{m}$ & 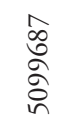 & 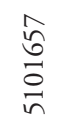 & 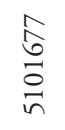 & 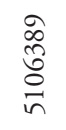 & 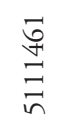 & $\begin{array}{l}\stackrel{\text { oे }}{ } \\
\text { o } \\
\text { O } \\
\text { in }\end{array}$ & 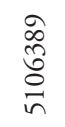 & $\begin{array}{l}\overrightarrow{+} \\
\tilde{n} \\
\tilde{o} \\
\tilde{n}\end{array}$ & 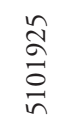 & $\begin{array}{l}\stackrel{m}{\approx} \\
\stackrel{\sigma}{n} \\
i\end{array}$ & 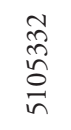 & \\
\hline
\end{tabular}

Diagnostic species of the association (Diagnostične vrste asociacije)

AP Primula carniolica

PcSp Phyteuma scheuchzeri subsp. columnae

AP Paederota lutea

AP Orthothecium rufescens

AP Valeriana tripteris

AP Carex brachystachys

AP Cystopteridion s. lat. (Astrantio-Paederotion luteae nom. prov.)

Aster bellidiastrum

Asplenium viride

Fissidens dubius

Valeriana saxatilis

Tofieldia calyculata

Viola biflora

Barbula crocea

Cystopteris fragilis

Preissia quadrata

$\begin{array}{lllllllllllll}\mathrm{E} 1 & 1 & + & \mathrm{r} & + & + & 1 & + & + & 1 & 1 & 2 & 2 \\ \mathrm{E} 1 & + & + & + & + & 1 & + & + & + & + & + & + & + \\ \mathrm{E} 1 & 1 & 1 & 1 & + & 1 & 1 & + & 1 & 2 & 1 & . & + \\ \mathrm{E} 0 & + & + & + & . & + & + & . & . & . & . & . & . \\ \mathrm{E} 1 & . & . & + & + & . & . & . & . & . & . & . & . \\ \mathrm{E} 1 & \mathrm{r} & . & . & . & 1 & + & . & . & . & . & . & .\end{array}$




\section{$\begin{array}{lllllllllllllllllllllllllll}50 & 51 & 52 & 53 & 54 & 55 & 56 & 57 & 58 & 59 & 60 & 61 & 62 & 63 & 64 & 65 & 66 & 67 & 68 & 69 & 70 & 71 & 72 & 73 & \text { Pr. } & \text { Fr. }\end{array}$}

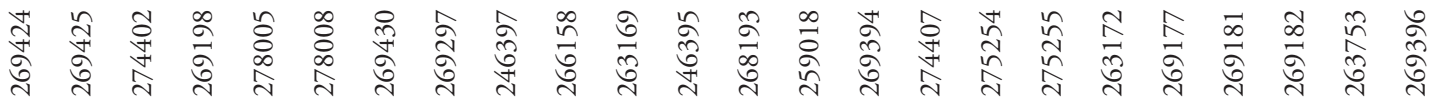

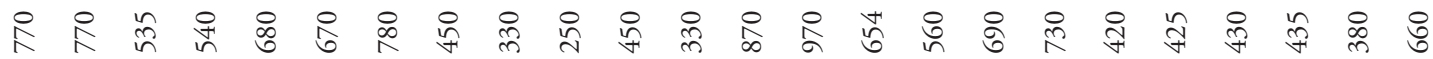

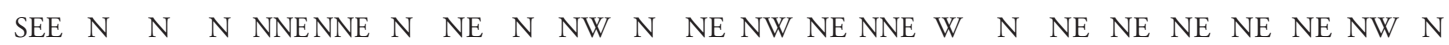
$\begin{array}{llllllllllllllllllllllll}85 & 80 & 70 & 70 & 80 & 80 & 80 & 85 & 90 & 100 & 90 & 80 & 85 & 90 & 80 & 80 & 85 & 90 & 80 & 80 & 60 & 80 & 80 & 90\end{array}$

$\begin{array}{lllllllllllllllllllllllllllllllll}\mathrm{D} & \mathrm{D} & \mathrm{D} & \mathrm{D} & \mathrm{D} & \mathrm{D} & \mathrm{D} & \mathrm{D} & \mathrm{D} & \mathrm{D} & \mathrm{D} & \mathrm{D} & \mathrm{D} & \mathrm{D} & \mathrm{D} & \mathrm{D} & \mathrm{D} & \mathrm{D} & \mathrm{D} & \mathrm{D} & \mathrm{D} & \mathrm{D} & \mathrm{D} & \mathrm{D}\end{array}$

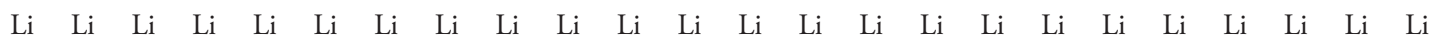

$\begin{array}{llllllllllllllllllllllll}100 & 100 & 100 & 100 & 100 & 100 & 100 & 100 & 100 & 100 & 100 & 100 & 100 & 100 & 100 & 100 & 100 & 100 & 100 & 100 & 100 & 100 & 100 & 100\end{array}$ $\begin{array}{llllllllllllllllllllllll}30 & 30 & 30 & 30 & 35 & 40 & 30 & 30 & 30 & 25 & 20 & 30 & 30 & 25 & 30 & 30 & 30 & 30 & 30 & 40 & 30 & 30 & 30 & 15\end{array}$ $\begin{array}{llllllllllllllllllllllll}40 & 40 & 30 & 50 & 10 & 20 & 30 & 30 & 30 & 25 & 19 & 20 & 30 & 20 & 10 & 30 & 30 & 30 & 30 & 60 & 5 & 20 & 20 & 10\end{array}$

$\begin{array}{llllllllllllllllllllllll}10 & 17 & 21 & 15 & 19 & 23 & 13 & 23 & 17 & 12 & 16 & 34 & 20 & 24 & 22 & 24 & 21 & 29 & 16 & 24 & 15 & 18 & 13 & 6\end{array}$

$\begin{array}{lllllllllllllllllllllllll}5 & 10 & 10 & 5 & 15 & 15 & 5 & 10 & 50 & 10 & 10 & 100 & 5 & 10 & 5 & 10 & 15 & 15 & 10 & 10 & 5 & 5 & 10 & 2\end{array}$

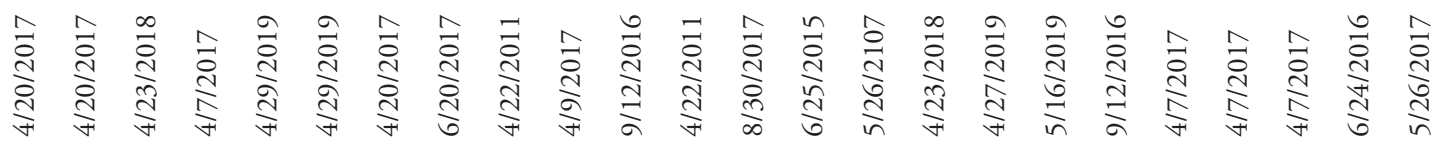

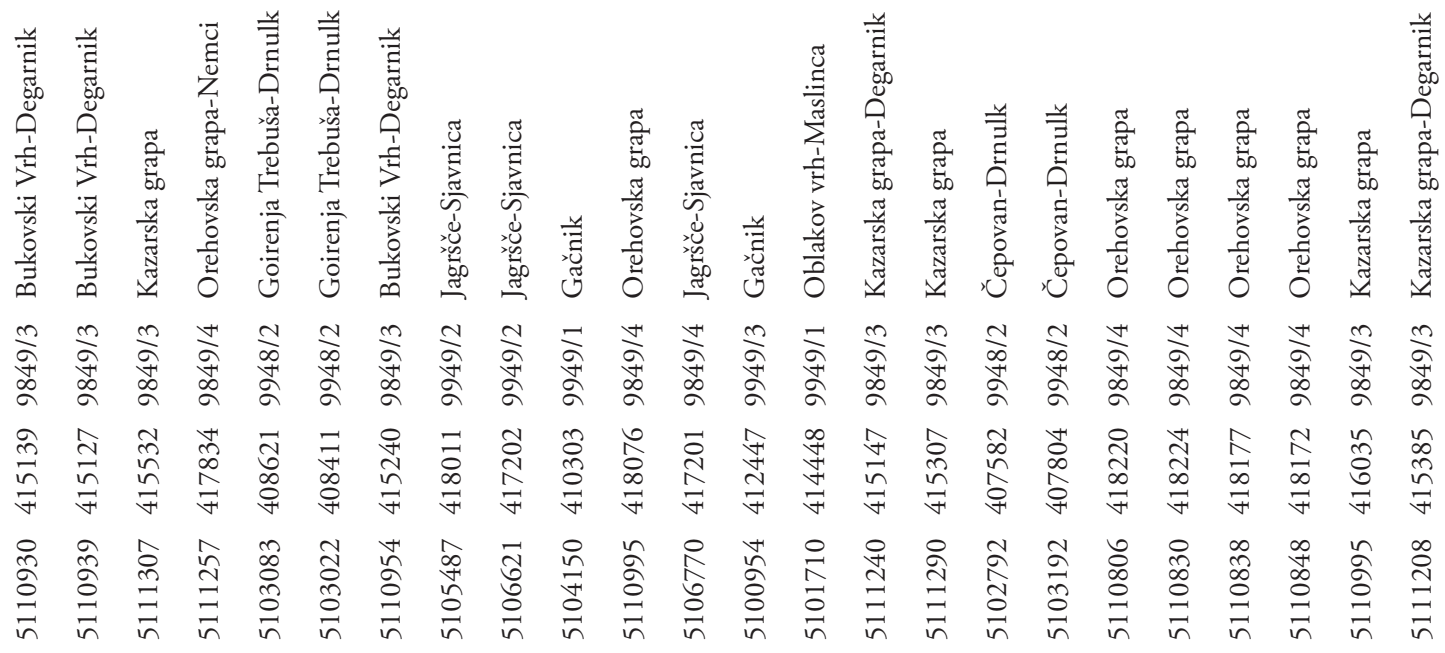

Pr. Fr. $\begin{array}{llllllllllllllllllllllllllllll}2 & 1 & 1 & 2 & 2 & 3 & 1 & 1 & 1 & 1 & 1 & 1 & 1 & 1 & 1 & + & 2 & 2 & 1 & 1 & 3 & 2 & + & 1 & 73 & 100\end{array}$ 73100 $54 \quad 74$ 4156 $36 \quad 49$ 
Hymenostylium recurvirostre

E0

Moehringia muscosa

Primula $x$ venusta

Pellia endiviifolia

Selaginella helvetica

Palustriella commutata

Heliosperma veselskyi subsp. veselskyi

PcSp Physoplexido comosae-Saxifragion petraeae

Campanula cespitosa

Athamanta turbith

Hieracium porrifolium

PC Potentilletalia caulescentis

Potentilla caulescens

Kernera saxatilis

Primula auricula

AT Asplenietea trichomanis

Asplenium ruta-muraria

Asplenium trichomanes

Hieracium glaucum

TR Thlaspietea rotundifolii

Hieracium bifidum

Gymnocarpium robertianum

Adenostyles glabra

Aquilegia iulia

Dryopteris villarii

MC Montio-Cardaminetea

Conocephalum conicum

Distichium capillaceum

Ditrichum flexicaule

Campylium stellatum

Cololejeunea calcarea

ES Elyno-Seslerietea

Sesleria caerulea

Carex mucronata

Betonica alopecuros

Carduus crassifolius

Carex ferruginea

Gentiana clusii

Laserpitium peucedanoides

FB Festuco-Brometea

Carex humilis

Buphthalmum salicifolium

Galium lucidum

Brachypodium rupestre

E1

E1

E0

E1

E0

E1

E1

E1

E1

E1

E1

E1

E1

E1

E1

E1

E1

E1

E1

E1

E0

E0

E0

E0

E0

E1

E1

E1

E1

E1

E1

E1

E1

E1

E1

E1

Angelica sylvestris

Caltha palustris

Taraxacum $x$ Ruderalia

BA Betulo-Alnetea

Salix appendiculata

Salix glabra

MuAMulgedio-Aconitetea

Senecio ovatus

Ranunculus platanifolius

Aconitum degenii subsp. paniculatum

Saxifraga rotundifolia

E1

E1

E1

E1

E1

E1

TG Trifolio-Geranietea

Laserpitium siler 
$\begin{array}{llllllllllllllllllllllllll}50 & 51 & 52 & 53 & 54 & 55 & 56 & 57 & 58 & 59 & 60 & 61 & 62 & 63 & 64 & 65 & 66 & 67 & 68 & 69 & 70 & 71 & 72 & 73 & \text { P.. } & \text { Fr. }\end{array}$

$\begin{array}{rr}6 & 8 \\ 6 & 8 \\ 3 & 4 \\ 2 & 3 \\ 2 & 3 \\ 1 & 1 \\ 1 & 1 \\ & \\ 11 & 15 \\ 4 & 5 \\ 4 & 5 \\ & \\ 7 & 10 \\ 5 & 7 \\ 1 & 1\end{array}$

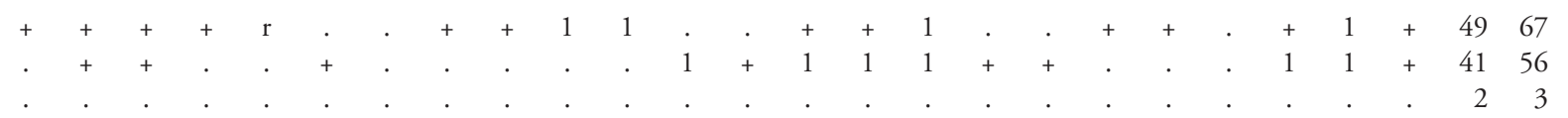
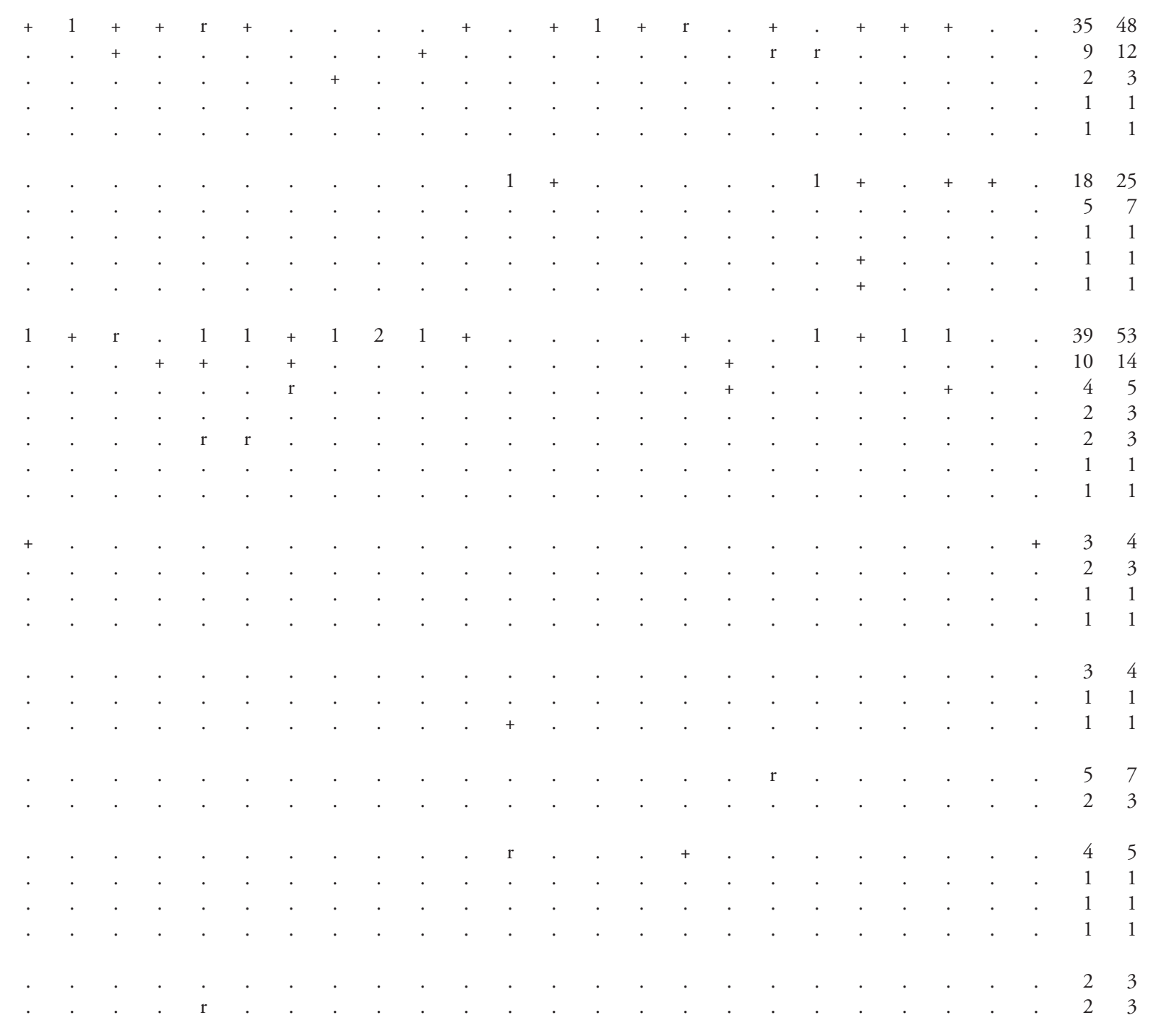
Anthericum ramosum

Laserpitium latifolium

Thalictrum minus

EA Epilobietea angustifolii

Rubus idaeus

EP Erico-Pinetea

Calamagrostis varia

Rhododendron hirsutum

Erica carnea

Polygala chamaebuxus

Carex ornithopoda

Rubus saxatilis

Carex alba

Cirsium erisithales

Leontodon incanus

Molinia arundinacea

Amelanchier ovalis

Aster amellus

Rhodothamnus chamaecistus

Aquilegia nigricans

Pinus sylvestris

VP Vaccinio-Pinetea

Veronica urticifolia

Homogyne sylvestris

Solidago virgaurea

Hieracium murorum

Clematis alpina

Gentiana asclepiadea

Oxalis acetosella

Picea abies

Polystichum lonchitis

Aposeris foetida

Rosa pendulina

AF Aremonio-Fagion

Cyclamen purpurascens

Helleborus niger

Hemerocallis lilioasphodelus

Potentilla carniolica

Cardamine trifolia

Primula vulgaris

Anemone trifolia

Euphorbia carniolica

Lamium orvala

Rhamnus fallax

Laserpitium krapfii

Omphalodes verna

Knautia drymeia

TA Tilio-Acerion

Aruncus dioicus

Polystichum aculeatum

Phyllitis scolopendrium

Acer pseudoplatanus

Geranium robertianum

Tephroseris pseudocrispa

Thalictrum aquilegiifolium

Ulmus glabra

Acer platanoides
E1

E1

E1

E1

E1

E1

E1

E1

E1

E1

E1

E1

E1

E1

E1

E1

E1

E1

E2a

E1

E1

E1

E1

E1

E1

E1

E1

E1

E1

E1

E1

E1

E1

E1

E1

E1

E1

E1

E1

E1

E1

E1

E1

E1

E1

E1 


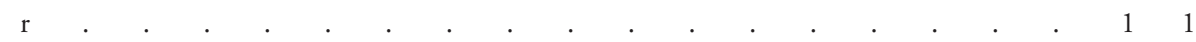


FS Fagetalia sylvaticae

Salvia glutinosa

Galeobdolon flavidum

Mercurialis perennis

Mycelis muralis

Galium laevigatum

Fagus sylvatica

Melica nutans

Symphytum tuberosum

Campanula trachelium

Cardamine pentaphyllos

Asarum europaeum subsp. caucasicum

Petasites albus

Sambucus nigra

Lathyrus vernus

QP Quercetalia pubescenti-petraeae

Fraxinus ornus

Ostrya carpinifolia

Mercurialis ovata

Clematis recta

Euonymus verrucosa

QF Querco-Fagetea

Carex digitata

Hepatica nobilis

Hedera helix

Veratrum nigrum

Acer campestre

Clematis vitalba

Quercus petraea

Cardamine impatiens

Lonicera xylosteum

E1

E1

E1

E1

E1

E1

E1

E1

E1

E1

E1

E1

E2a

E1

E1

E1

E1

E1

E2a

E1

E1

E1

E1

E1

E1

E1

E1

E1

ML Mosses and lichens (Mahovi in lišaji)

Neckera crispa

Ctenidium molluscum

Tortella tortuosa

Mnium thomsonii

Schistidium apocarpum

Plagiochila porreloides

Encalypta streptocarpa

Leiocolea collaris

Marchantia polymorpha

Bryum capillare

Pedinophyllum interruptum

Mnium stellare

Isothecium alopecuroides

Lejeunea cavifolia

Myurella sibirica

Neckera complanata

Didymodon ferrugineus

Trichostomum brachydontium

Myurella julacea

Hypnum cupressiforme var. resupinatum

Lichenes div.

Lophozia sp.

Dichodontium pellucidum subsp. flavescens

Brachythecium rutabulum

Plagiomnium rostratum

Mnium marginatum

Plagiobryum zierii

E0

E0

E0

E0

E0

E0

E0

E0

E0

E0

E0

E0

E0

E0

E0

E0

E0

E0

E0

E0

E0

E0

E0

E0

E0

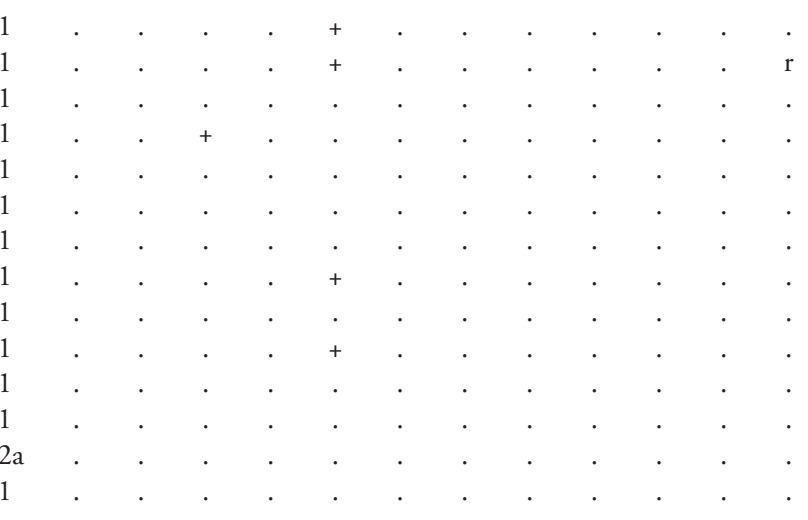

$\mathrm{E} 01++\quad+\quad 2 \quad \begin{array}{lllllll}1 & 1 & 1 & 1 & 1 & 2\end{array}$

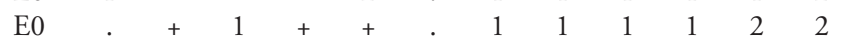

Legend / Legenda: D Dolomite - dolomit L Limestone-apnenec Li Lithosol-kamnišče Fr. Frequency in \%-frekvenca v \% 


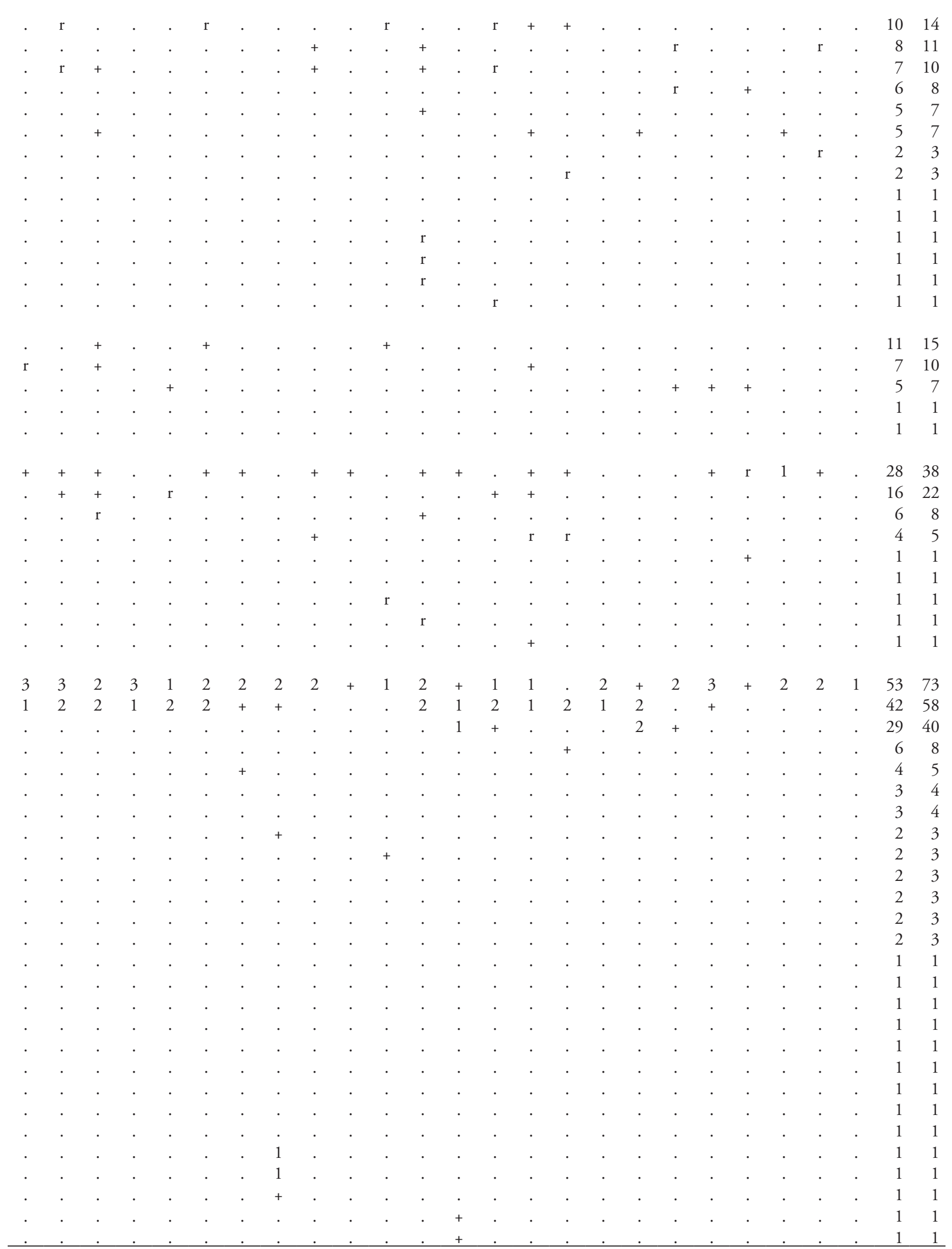


Table 6 (Tabela 6): Phyteumato-Primuletum carniolicae astrantietosum carniolicae.

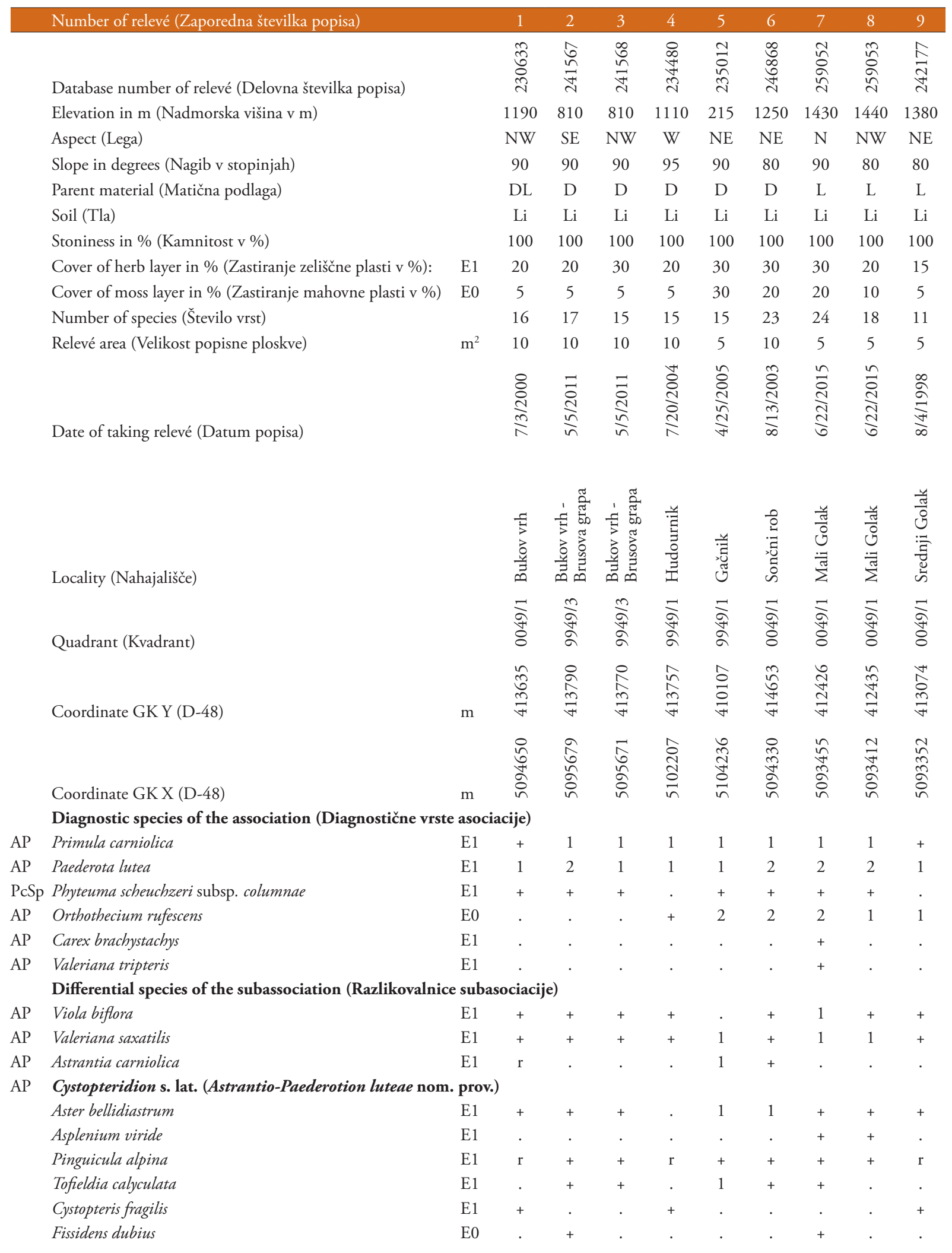




\begin{tabular}{|c|c|c|c|c|c|c|c|c|c|c|c|c|c|c|c|c|c|}
\hline 10 & 11 & 12 & 13 & 14 & 15 & 16 & 17 & 18 & 19 & 20 & 21 & 22 & 23 & 24 & 25 & 26 & 27 \\
\hline 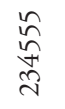 & $\begin{array}{l}\stackrel{n}{\cong} \\
\stackrel{\Xi}{\approx}\end{array}$ & $\begin{array}{l}\stackrel{n}{n} \\
\stackrel{\Xi}{\approx}\end{array}$ & 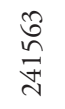 & $\begin{array}{l}\hat{O} \\
\& \\
O \\
\stackrel{+}{N}\end{array}$ & 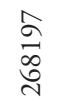 & 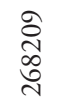 & $\underset{\hat{\aleph}}{\stackrel{\widehat{N}}{\hat{~}}}$ & 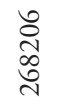 & 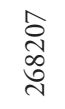 & 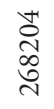 & $\frac{\stackrel{\sim}{\approx}}{\stackrel{\sim}{\sim}}$ & $\begin{array}{l}\stackrel{n}{\hat{N}} \\
\stackrel{\sim}{ }\end{array}$ & $\begin{array}{l}\stackrel{N}{N} \\
\underset{0}{0} \\
\stackrel{\sim}{N}\end{array}$ & $\begin{array}{l}\stackrel{\infty}{ \pm} \\
\underset{\sim}{n} \\
\stackrel{+}{\sim}\end{array}$ & 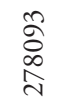 & 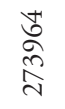 & 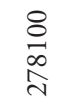 \\
\hline 810 & 420 & 430 & 800 & 1250 & 890 & 845 & 775 & 845 & 890 & 850 & 590 & 755 & 840 & 430 & 470 & 780 & 460 \\
\hline $\mathrm{NE}$ & $\mathrm{N}$ & NW & $\mathrm{NE}$ & $\mathrm{N}$ & E & SE & $\mathrm{N}$ & $\mathrm{E}$ & NW & W & W & $\mathrm{NE}$ & NW & SE & SW & $\mathrm{NE}$ & $\mathrm{N}$ \\
\hline 80 & 90 & 95 & 80 & 80 & 95 & 80 & 85 & 80 & 80 & 90 & 95 & 95 & 85 & 90 & 90 & 80 & 80 \\
\hline $\mathrm{D}$ & $\mathrm{D}$ & $\mathrm{D}$ & $\mathrm{D}$ & $\mathrm{D}$ & $\mathrm{D}$ & $\mathrm{D}$ & $\mathrm{D}$ & D & D & $\mathrm{D}$ & $\mathrm{D}$ & $\mathrm{D}$ & $\mathrm{D}$ & $\mathrm{D}$ & $\mathrm{D}$ & $\mathrm{D}$ & $\mathrm{D}$ \\
\hline $\mathrm{Li}$ & $\mathrm{Li}$ & $\mathrm{Li}$ & $\mathrm{Li}$ & $\mathrm{Li}$ & $\mathrm{Li}$ & $\mathrm{Li}$ & $\mathrm{Li}$ & $\mathrm{Li}$ & $\mathrm{Li}$ & $\mathrm{Li}$ & $\mathrm{Li}$ & $\mathrm{Li}$ & $\mathrm{Li}$ & $\mathrm{Li}$ & $\mathrm{Li}$ & $\mathrm{Li}$ & $\mathrm{Li}$ \\
\hline 100 & 100 & 100 & 100 & 100 & 100 & 100 & 100 & 100 & 100 & 100 & 100 & 100 & 100 & 100 & 100 & 100 & 100 \\
\hline 40 & 20 & 35 & 30 & 35 & 20 & 30 & 30 & 40 & 60 & 30 & 15 & 20 & 35 & 30 & 30 & 60 & 60 \\
\hline 10 & 10 & 10 & 10 & 20 & 10 & 10 & 20 & 30 & 30 & 20 & 5 & 20 & 20 & 10 & 30 & 30 & 70 \\
\hline 22 & 18 & 16 & 24 & 22 & 16 & 18 & 17 & 18 & 24 & 21 & 18 & 17 & 22 & 14 & 17 & 17 & 20 \\
\hline 5 & 10 & 10 & 10 & 10 & 5 & 5 & 20 & 5 & 5 & 5 & 20 & 10 & 5 & 15 & 15 & 10 & 15 \\
\hline 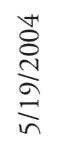 & $\frac{\infty}{\frac{\infty}{\infty}}$ & $\frac{\infty}{\stackrel{\infty}{\infty}}$ & 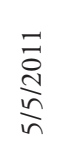 & 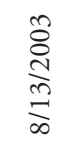 & 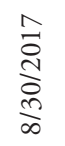 & 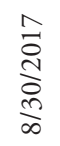 & $\underset{\substack{\infty \\
\stackrel{\infty}{N}}}{\stackrel{\infty}{\sim}}$ & 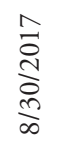 & 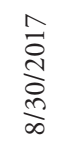 & 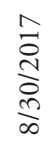 & 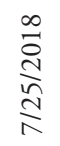 & $\frac{\infty}{\stackrel{\infty}{a}}$ & 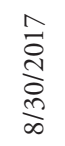 & 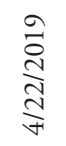 & 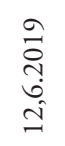 & 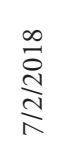 & 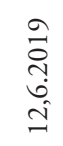 \\
\hline
\end{tabular}

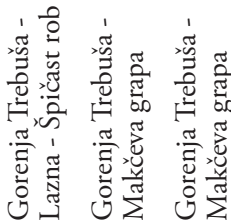

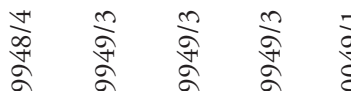

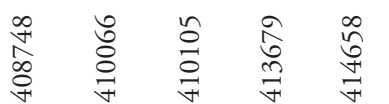

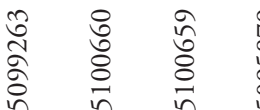

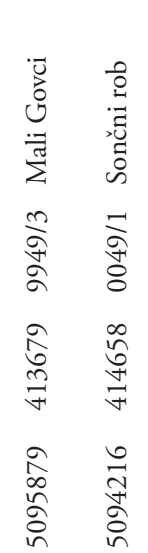

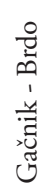

$\begin{array}{ll}\stackrel{n}{\sigma} & \frac{\pi}{\sigma} \\ \sigma & \sigma\end{array}$

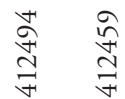

$\begin{array}{ll}\infty & 0 \\ \stackrel{0}{*} & \stackrel{0}{\circ} \\ \sim & i\end{array}$

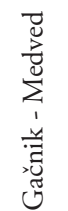

市

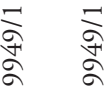

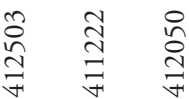

în

Pr. Fr.

Pr. Fr.

27100

27100

$22 \quad 81$

1970

$13 \quad 48$

$10 \quad 37$

2593

2281

$18 \quad 67$ 
Cystopteris regia

Hymenostylium recurvirostre

Pellia endiviifolia

Barbula crocea

Solorina saccata

Heliosperma pusillum

Preissia quadrata

Palustriella commutata

PcSp Physoplexido comosae-Saxifragion petraeae

Campanula cespitosa

PC Potentilletum caulescentis

Potentilla caulescens

Kernera saxatilis

AT Asplenietea trichomanis

Asplenium ruta-muraria

Asplenium trichomanes

TR Thlapietea rotundifolii

Adenostyles glabra

Hieracium bifidum

Dryopteris villarii

Aquilegia iulia

Gymnocarpium robertianum

CD Caricetalia davallianae

Parnassia palustris

MC Montio-Cardaminetea

Conocephalum conicum

Campylium stellatum

Distichium capillaceum

ES Elyno-Seslerietea

Sesleria caerulea

Laserpitium peucedanoides

Carex ferruginea

Carex mucronata

Carex firma

Campanula witasekiana

Phyteuma orbiculare

TG Trifolio-Geranietea

Digitalis grandiflora

$\begin{array}{llllllllll}\text { E1 } & + & + & + & + & + & + & \text { r } & . & . \\ \text { E1 } & . & . & \text { r } & . & . & . & + & + & . \\ \text { E1 } & + & . & . & . & . & . & . & . & . \\ \text { E1 } & . & + & \text { r } & + & . & . & . & . & . \\ \text { E1 } & . & . & \text { r } & . & . & 1 & . & . & . \\ \text { E1 } & . & . & . & . & . & . & + & . & . \\ \text { E1 } & . & . & . & . & . & . & . & . & .\end{array}$

BA Betulo-Alnetea

Salix appendiculata

MuA Mulgedio-Aconitetea

Chaerophyllum hirsutum

E1

Veratrum album

Crepis paludosa

EA Epilobietea angustifolii

Rubus idaeus

EP Erico-Pinetea

Calamagrostis varia

Rhododendron hirsutum

Rhodothamnus chamaecistus 
Polygala chamaebuxus

Erica carnea

Cirsium erisithales

Aquilegia nigricans

Molinia arundinacea

VP Vaccinio-Piceetea

Homogyne sylvestris

Clematis alpina

Veronica urticifolia

AF Aremonio-Fagion

Anemone trifolia

Cyclamen purpurascens

Cardamine enneaphyllos

Cardamine trifolia

TA Tilio-Acerion

Acer pseudoplatanus

Aruncus dioicus

Thalictrum aquilegiifolium

FS Fagetalia sylvaticae

Galeobdolon flavidum

Fagus sylvatica

Galium laevigatum

Mycelis muralis

Salvia glutinosa

QP Quercetalia pubescentis

Fraxinus ornus

Ostrya carpinifolia

E1

\section{Querco-Fagetea}

Carex digitata

Hepatica nobilis

\section{E1}

E1

E1

E1

E1

Carex umbrosa

\section{E1}

E2a

E1

E1

E1

ML Mosses and lichens (Mahovi in lišaji)

Ctenidium molluscum

E0

Neckera crispa

E0

Tortella tortuosa

E0

Brachythecium rutabulum

E0

Plagiopus oederi

Bryum sp.

E0

Lescuraea plicata

E0

Dicranum scoparium

E0

Marchantia polymorpha

E0

Schistidium apocarpum

E0

Pedinophyllum interruptum

E0

Plagiochila porelloides

E0

E0

\section{Legend / Legenda}

D Dolomite-dolomit

L Limestone - apnenec

Li Lithosol - kamnišče

Pr. Presence (number of relevés in which the species is presented) - število popisov, v katerih se pojavlja vrsta

Fr. Frequency in $\%$ - frekvenca $\mathrm{\%}$ 


\begin{tabular}{|c|c|c|c|c|c|c|c|c|c|c|c|c|c|c|c|c|c|c|c|c|}
\hline & . & . & . & . & . & . & . & . & . & . & . & . & . & . & . & . & . & & 1 & 4 \\
\hline & . & & . & . & . & . & . & . & & . & . & . & . & . & . & & & & 1 & 4 \\
\hline & . & . & . & . & . & . & . & . & . & . & . & . & & . & . & . & . & & 1 & 4 \\
\hline+ & . & . & . & . & . & . & . & . & . & . & . & . & . & . & . & . & . & & 1 & 4 \\
\hline & . & . & . & . & . & $\mathrm{r}$ & . & . & . & . & . & . & & . & . & . & . & & 1 & 4 \\
\hline & . & . & . & . & . & . & . & . & . & . & . & . & . & . & . & . & 1 & & 1 & 4 \\
\hline+ & $\mathrm{r}$ & . & . & . & . & . & + & . & $\mathrm{r}$ & . & . & . & . & . & . & . & . & & 4 & 15 \\
\hline & . & . & . & . & . & . & . & . & + & . & . & + & 2 & . & . & + & . & & 4 & 15 \\
\hline . & . & . & . & . & . & . & . & . & . & . & . & . & & . & . & . & 1 & & 1 & 4 \\
\hline+ & + & . & . & . & . & . & . & . & . & . & . & . & & . & . & . & . & & 2 & 7 \\
\hline & . & . & . & . & + & . & . & . & . & . & . & + & . & . & . & . & . & & 2 & 7 \\
\hline . & . & . & $\mathrm{r}$ & . & . & . & . & . & . & . & . & . & . & . & . & . & . & & 1 & 4 \\
\hline$\cdot$ & . & . & . & . & . & . & . & . & . & . & . & . & . & + & . & . & . & & 1 & 4 \\
\hline . & . & . & . & . & . & . & . & . & $\mathrm{r}$ & . & . & . & . & . & . & . & . & & 2 & 7 \\
\hline . & . & . & . & . & . & . & . & . & . & . & . & . & + & . & . & . & . & & 1 & 4 \\
\hline . & . & . & . & . & . & . & . & . & . & . & . & . & & . & . & . & + & & 1 & 4 \\
\hline & + & + & . & . & . & . & . & . & . & + & . & . & & . & . & . & . & & 3 & 11 \\
\hline r & . & . & . & . & . & . & . & . & . & . & . & . & + & . & . & . & . & & 2 & 7 \\
\hline$\cdot$ & . & . & . & . & . & . & + & . & . & . & . & . & . & . & . & . & + & & 2 & 7 \\
\hline . & . & . & . & . & . & . & . & . & . & + & + & . & . & . & . & . & . & & 2 & 7 \\
\hline & . & . & $\mathrm{r}$ & . & . & . & . & . & . & . & . & . & . & . & . & . & . & & 1 & 4 \\
\hline & + & & + & . & . & . & . & . & . & . & . & . & . & . & . & & . & & 2 & 7 \\
\hline & . & . & + & . & . & . & . & . & . & . & . & . & . & . & . & . & . & & 1 & 4 \\
\hline 1 & $\mathrm{r}$ & + & . & . & . & $\mathrm{r}$ & . & . & $r$ & + & . & + & + & . & + & . & . & & 9 & 33 \\
\hline & . & . & . & . & . & . & . & . & . & . & . & . & 2 & + & . & . & . & & 2 & 7 \\
\hline . & . & . & . & . & . & . & . & . & . & . & . & . & . & . & . & . & . & & 1 & 4 \\
\hline+ & + & . & 1 & 1 & + & 1 & 1 & . & 1 & . & + & . & + & 1 & 1 & . & . & & 14 & 52 \\
\hline 1 & + & 1 & 1 & 1 & + & 1 & . & 1 & + & . & . & . & 1 & . & . & . & . & & 11 & 41 \\
\hline & . & & . & + & . & 1 & . & + & 2 & + & . & + & 1 & . & . & 1 & . & & 9 & 33 \\
\hline & . & . & . & . & . & . & . & . & . & . & . & . & & . & 1 & 1 & 2 & & 3 & 11 \\
\hline & . & . & . & . & . & . & . & . & . & . & . & . & . & . & . & . & . & & 2 & 7 \\
\hline & . & . & . & . & . & . & . & . & . & . & . & . & . & . & . & & . & & 1 & 4 \\
\hline & . & . & . & . & . & . & . & . & . & . & . & . & & . & . & . & . & & 1 & 4 \\
\hline & . & . & . & . & . & . & . & . & . & . & . & . & . & . & . & . & . & & 1 & 4 \\
\hline & . & . & + & . & . & . & . & . & . & . & . & . & . & . & . & . & . & & 1 & 4 \\
\hline & . & . & . & + & . & . & . & . & . & . & . & . & . & . & . & . & . & & 1 & 4 \\
\hline & . & . & . & . & . & . & . & . & . & . & + & . & & . & . & . & . & & 1 & 4 \\
\hline & & & & & & & & & & & & & & & & & & & 1 & \\
\hline
\end{tabular}




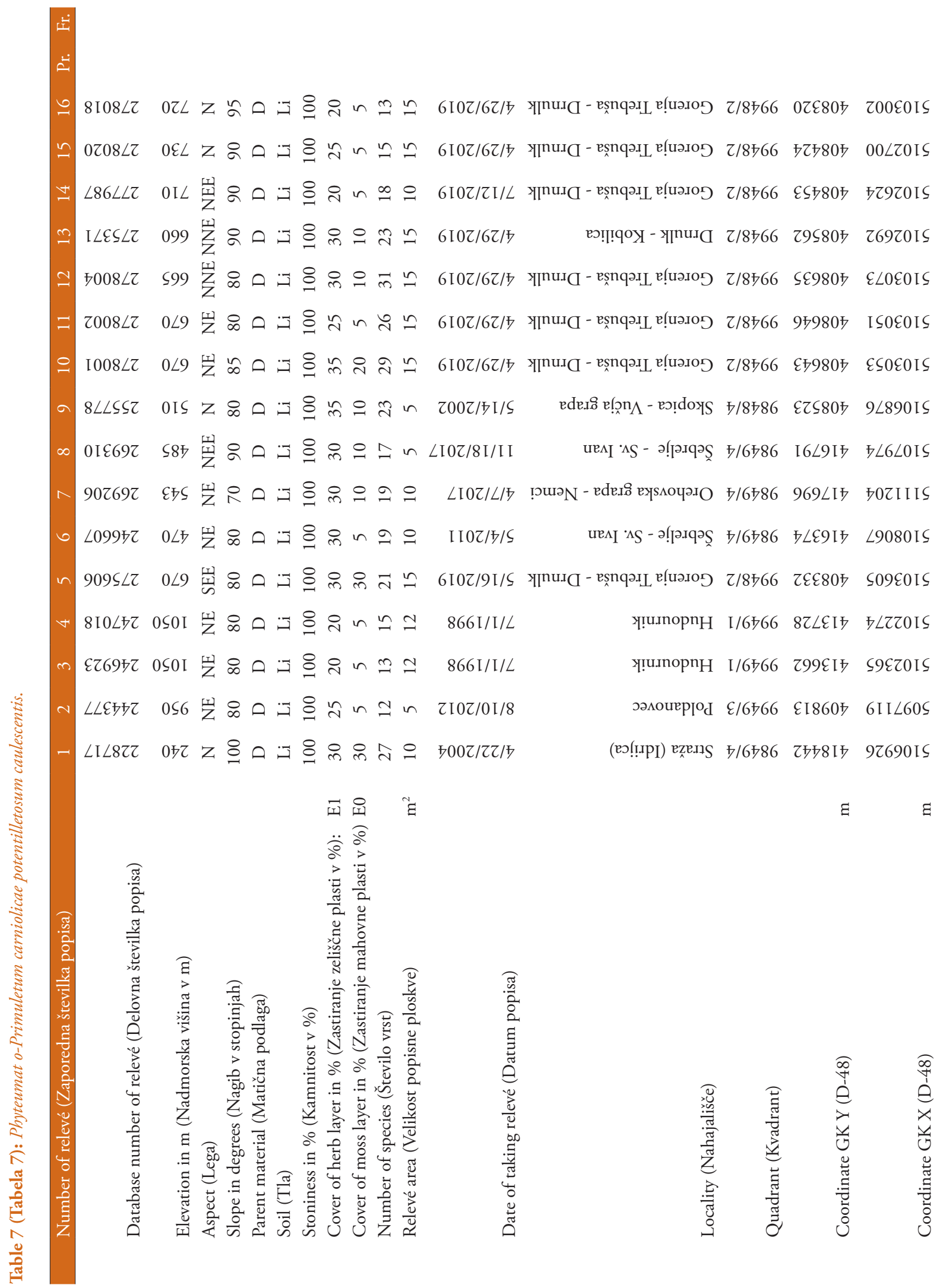


庄

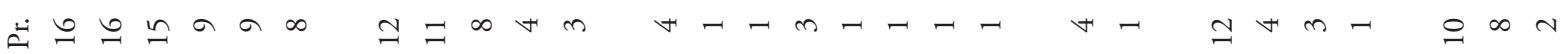
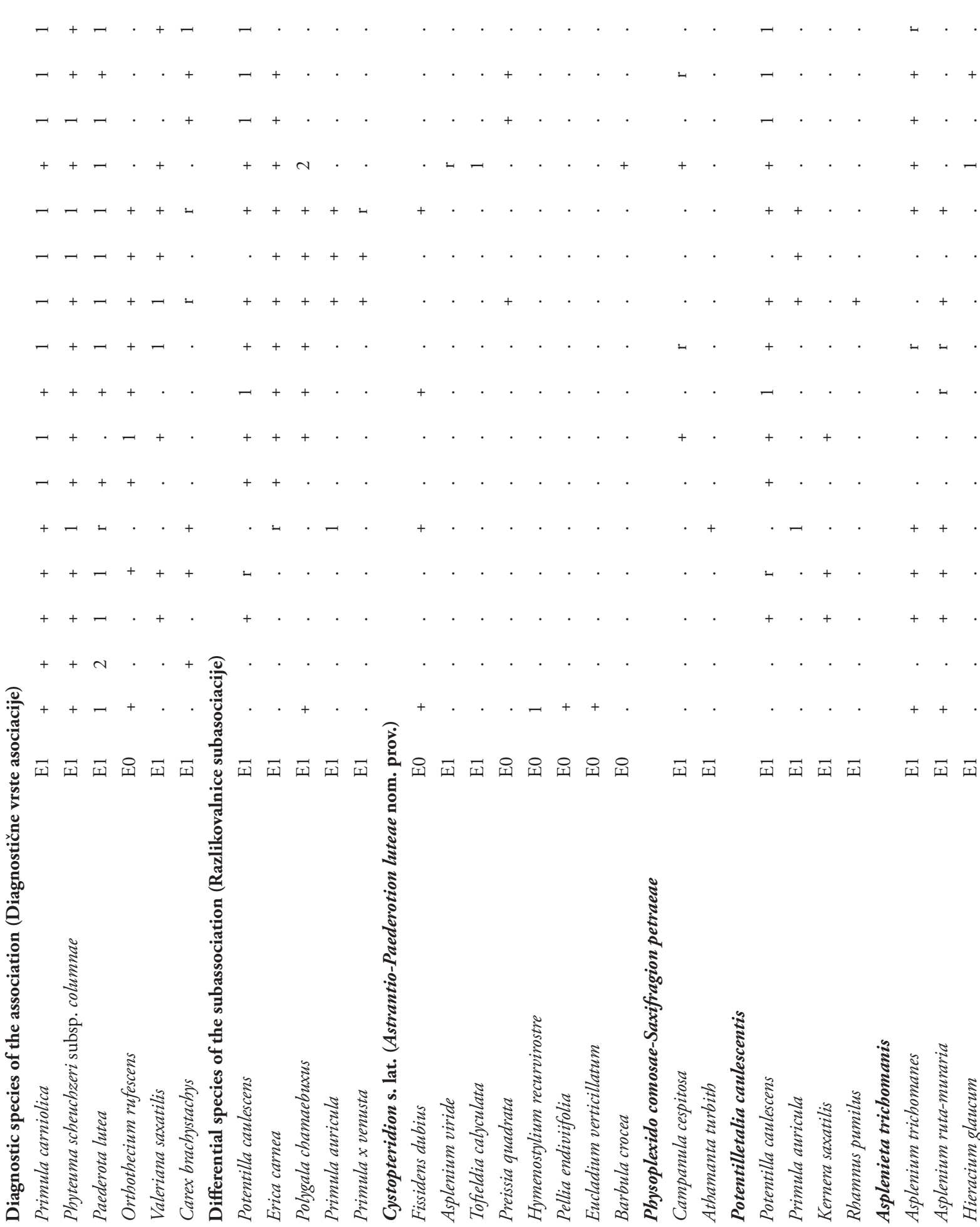

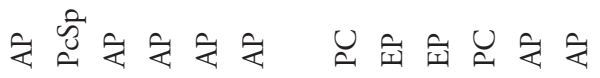
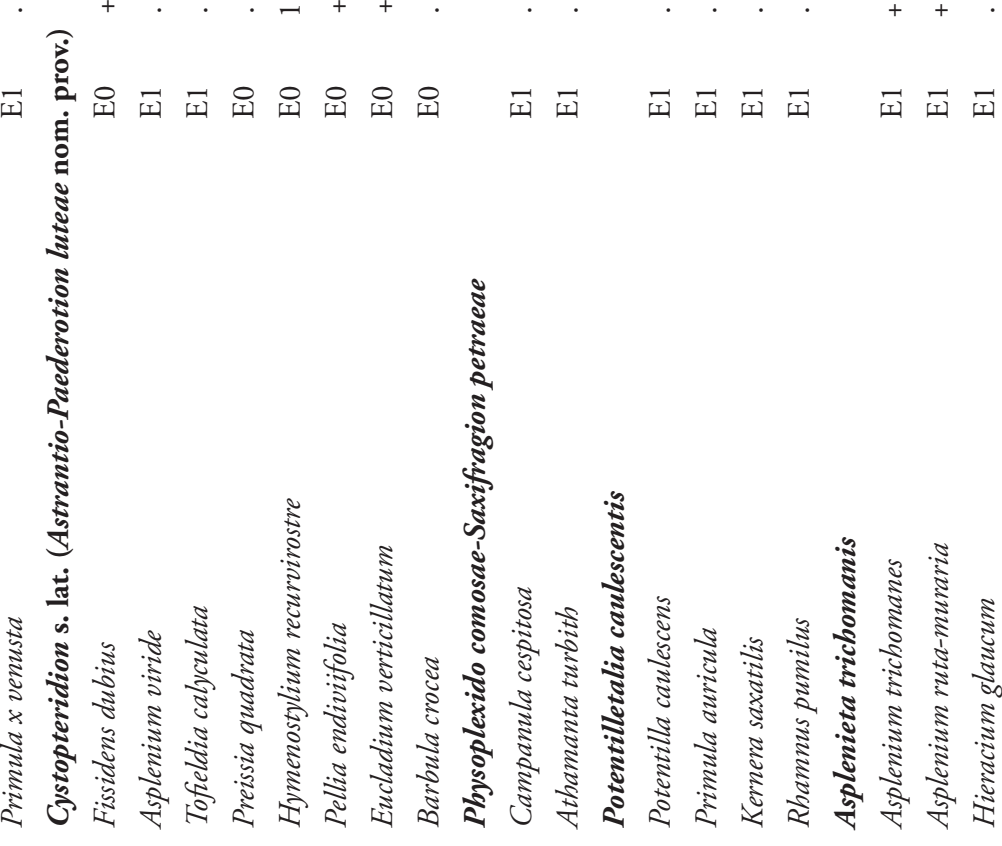

के

$\circlearrowleft$

安 


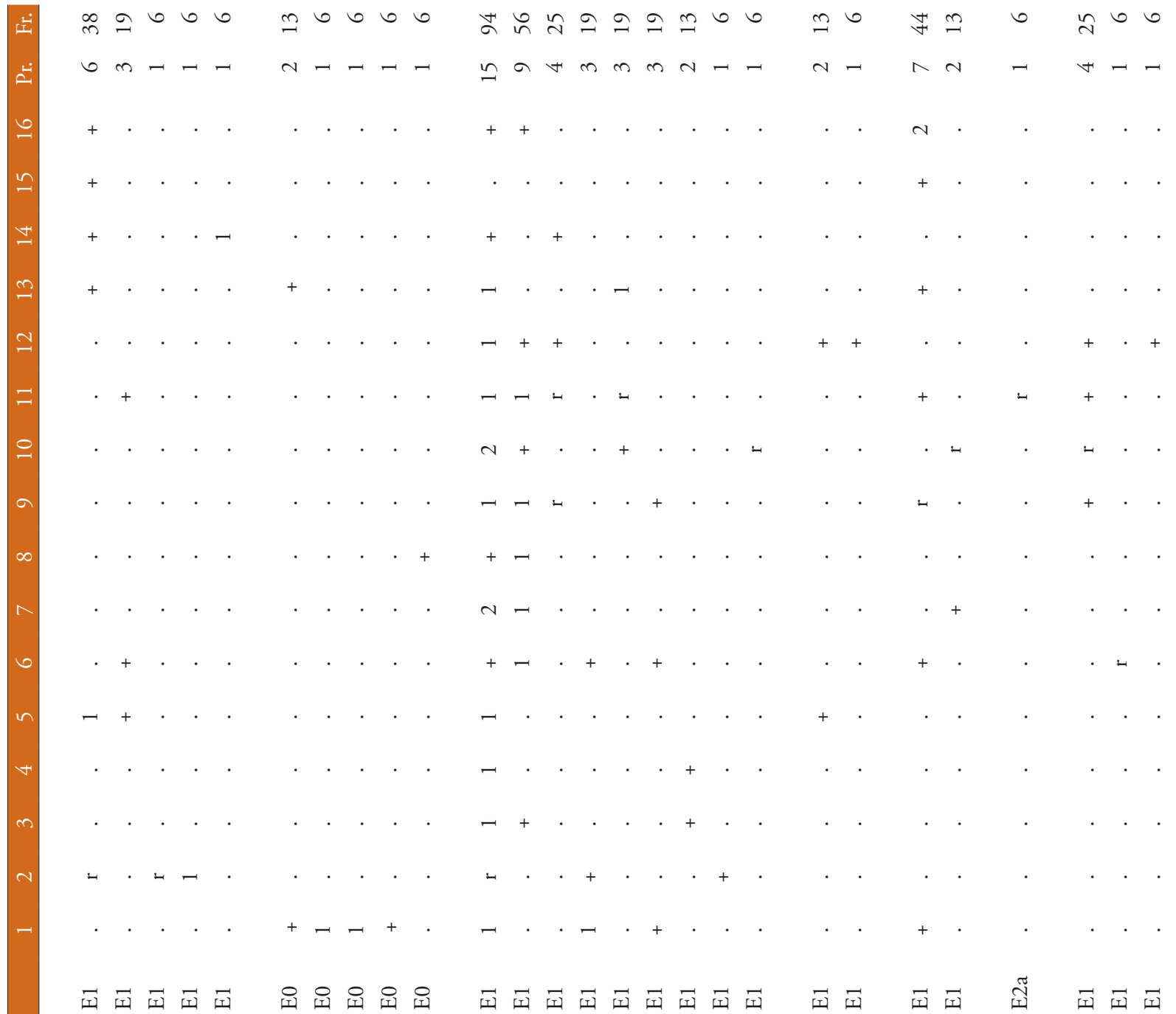




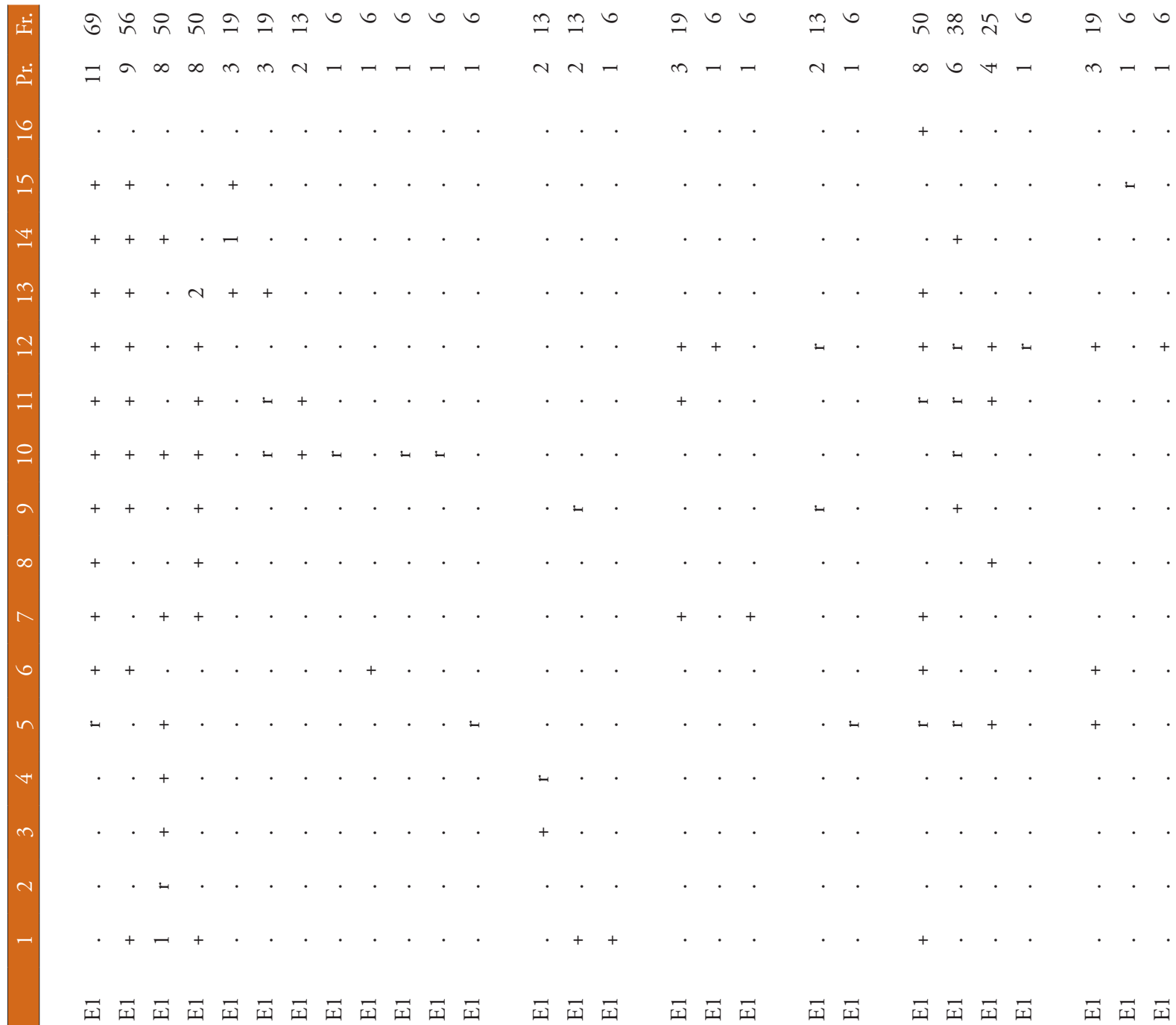




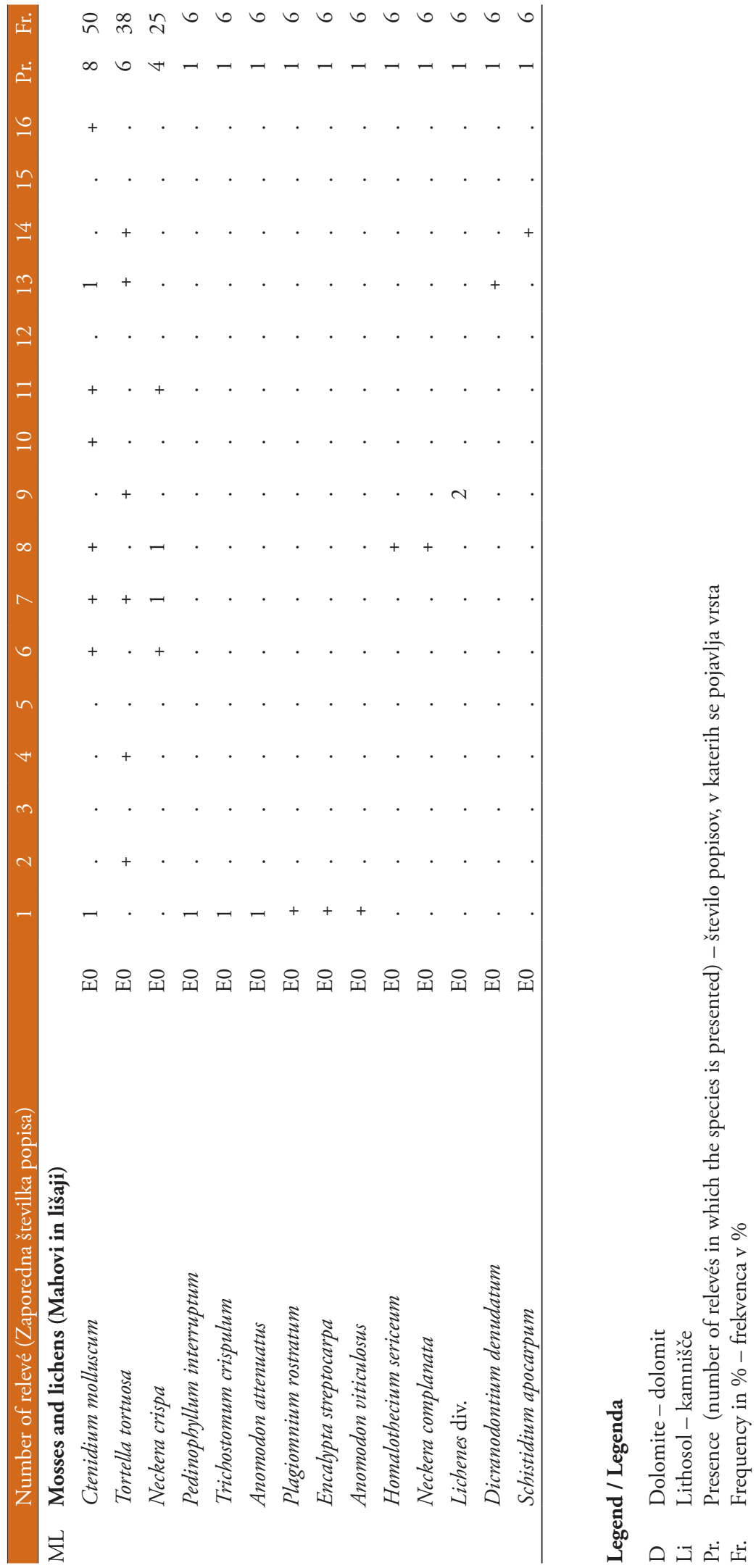


Table 8 (Tabela 8): Primulo carniolicae-Potentilletum clusianae.

Number of relevé (Zaporedna številka popisa)

Database number of relevé (Delovna številka popisa)

Elevation in $\mathrm{m}$ (Nadmorska višina $\mathrm{v} \mathrm{m}$ )

Aspect (Lega)

Slope in degrees (Nagib v stopinjah)

Parent material (Matična podlaga)

Soil (Tla)

Stoniness in \% (Kamnitost v \%)

Cover of herb layer in \% (Zastiranje zeliščne plasti v \%): $\quad \mathrm{E} 1$

Cover of moss layer in \% (Zastiranje mahovne plasti v \%) E0

Number of species (Število vrst)

Relevé area (Velikost popisne ploskve)

Date of taking relevé (Datum popisa)

Locality (Nahajališče)

Quadrant (Kvadrant)

Coordinate GK Y (D-48)

Coordinate GK X (D-48)

Diagnostic species of the association (Diagnostične vrste asociacije)

$\begin{array}{ll}\text { PC } & \text { Potentilla clusiana } \\ \text { AP } & \text { Primula carniolica } \\ \text { ES } & \text { Carex firma } \\ \text { EP Rhodothamnus chamaecistus } \\ \text { PC Campanula cochleariifolia } \\ \text { TR Hladnikia pastinacifolia } \\ \text { TR Aquilegia iulia } \\ \text { ES Hieracium pilosum }\end{array}$

AP Cystopteridion s. lat. (Astrantio-Paederotion luteae nom. prov.)

Paederota lutea

Valeriana saxatilis

Astrantia carniolica

Viola biflora

Tofieldia calyculata

Carex brachystachys

Orthothecium rufescens

Pinguicula alpina

Ranunculus traunfellneri

Soldanella minima

Primula $x$ venusta

Cystopteris fragilis

\section{$1 \quad 2$ \\ 3 \\ 4 \\ $5 \quad 6$ Pr. $\quad$ Fr.}

$\begin{array}{lllllll}236342 & 234471 & 246510 & 236341 & 236351 & 236350\end{array}$

$\begin{array}{llllll}1320 & 1280 & 1170 & 1330 & 1325 & 1320\end{array}$

NW NW NE NW NW NE

$\begin{array}{llllll}90 & 90 & 85 & 90 & 90 & 90\end{array}$

$\begin{array}{llllll}\mathrm{D} & \mathrm{D} & \mathrm{D} & \mathrm{D} & \mathrm{D} & \mathrm{D}\end{array}$

$\begin{array}{llllll}\mathrm{Li} & \mathrm{Li} & \mathrm{Li} & \mathrm{Li} & \mathrm{Li} & \mathrm{Li}\end{array}$

$\begin{array}{llllll}100 & 100 & 100 & 70 & 70 & 100\end{array}$

$\begin{array}{llllll}30 & 30 & 30 & 30 & 30 & 30\end{array}$

$\begin{array}{llllll}5 & 5 & 5 & 5 & 5 & 5\end{array}$

$\begin{array}{llllll}13 & 21 & 23 & 18 & 21 & 22\end{array}$

$\begin{array}{lllllll}\mathrm{m}^{2} & 2 & 10 & 10 & 5 & 3 & 10\end{array}$

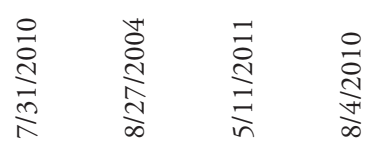

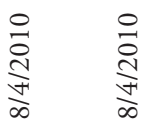

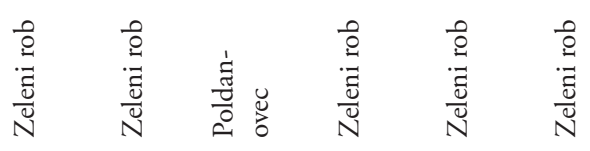

$\begin{array}{llllll}9949 / 3 & 9949 / 3 & 9949 / 3 & 9949 / 3 & 9949 / 3 & 9949 / 3\end{array}$

m $411517 \quad 411773 \quad 410299411442 \quad 411445 \quad 411512$

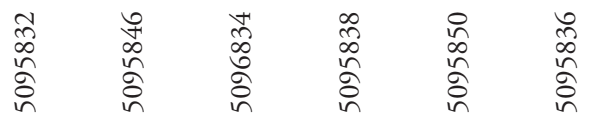

Pr. Fr.

$\begin{array}{rrrrrrrrr}\mathrm{E} 1 & + & 1 & 1 & 2 & 1 & + & 6 & 100 \\ \mathrm{E} 1 & + & + & + & 1 & 1 & 1 & 6 & 100 \\ \mathrm{E} 1 & + & 1 & 1 & + & + & + & 6 & 100 \\ \mathrm{E} 1 & + & 1 & 1 & + & + & + & 6 & 100 \\ \mathrm{E} 1 & + & \mathrm{r} & \cdot & + & + & 1 & 5 & 83 \\ \mathrm{E} 1 & . & + & + & \mathrm{r} & \cdot & \cdot & 3 & 50 \\ \mathrm{E} 1 & + & . & + & . & \mathrm{r} & + & 3 & 50 \\ \mathrm{E} 1 & + & + & + & + & + & + & 3 & 50\end{array}$

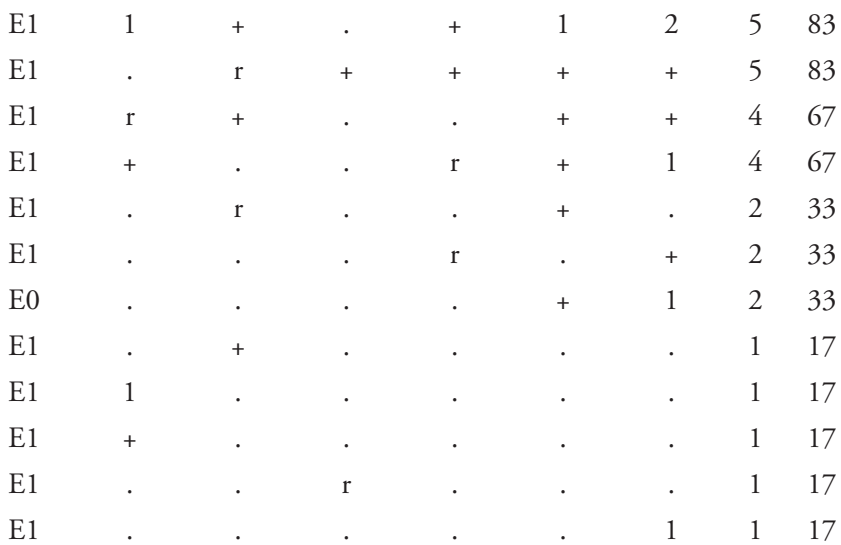


Number of relevé (Zaporedna številka popisa)

\section{1}

3

4

5

6 Pr. Fr.

PcSp Physoplexido comosae-Saxifragion petraeae

Phyteuma scheuchzeri subsp. columnae

E1

Saxifraga squarrosa

$$
\text { E1 }
$$

PC Potentilletalia caulescentis

Bupleurum petraeum

\section{E1}

Primula auricula

$$
\text { E1 }
$$

Rhamnus pumilus

Valeriana tripteris

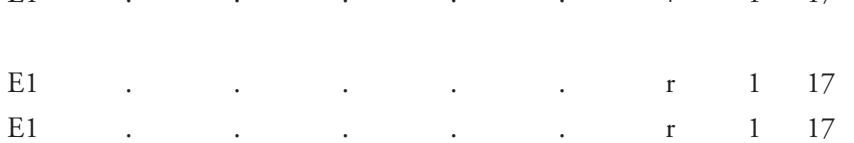

MC Montio-Cardaminetea

Distichium capillaceum

ES Elyno-Seslerietea

Sesleria caerulea

Aster bellidiastrum

Carex mucronata

Laserpitium peucedanoides

Hieracium pilosum

Hieracium villosum

Leontopodium alpinum

$\begin{array}{llllllllr}\text { E1 } & \text { r } & 1 & 1 & 2 & 1 & 1 & 6 & 100 \\ \text { E1 } & + & \cdot & \cdot & \cdot & + & + & 3 & 50 \\ \text { E1 } & \cdot & . & + & + & + & \cdot & 3 & 50 \\ \text { E1 } & . & . & \text { r } & + & + & . & 3 & 50 \\ \text { E1 } & . & . & . & + & + & + & 3 & 50 \\ \text { E1 } & . & \text { r } & . & . & . & . & 1 & 17 \\ \text { E1 } & . & . & + & . & . & . & 1 & 17 \\ \text { E1 } & . & . & + & . & . & . & 1 & 17 \\ \text { E1 } & . & . & \text { r } & . & . & . & 1 & 17\end{array}$

\section{BA Betulo-Alnetea}

Salix appendiculata

Salix glabra

$\begin{array}{lllllllll}\text { E1 } & . & \mathrm{r} & \mathrm{r} & \cdot & \cdot & \cdot & 2 & 33 \\ \mathrm{E} 1 & \cdot & + & + & \cdot & \cdot & \cdot & 2 & 33 \\ & & & & & & & & \\ \mathrm{E} 1 & . & + & + & . & + & + & 4 & 67 \\ \mathrm{E} 1 & . & . & \mathrm{r} & . & . & . & 1 & 17\end{array}$

ML Mosses (Mahovi)

Neckera crispa

\begin{tabular}{|c|c|c|c|c|c|c|c|}
\hline E0 & . & + & + & 1 & . & . & 3 \\
\hline E0 & . & + & + & + & + & 1 & 5 \\
\hline E0 & + & + & . & . & . & . & 2 \\
\hline
\end{tabular}

Tortella tortuosa

\section{Legend / Legenda}

D Dolomite - dolomit

Li Lithosol - kamnišče

Pr. Presence (number of relevés in which the species is presented) - število popisov, v katerih se pojavlja vrsta

Fr. Frequency in $\%$ - frekvenca v \% 
Table 9: Synoptic table of rock crevice communities with Primula carniolica.

Tabela 9: Sintezna tabela združb skalnih razpok z vrsto Primula carniolica.

\begin{tabular}{llllllllllllllll}
\hline Successive number (Zaporedna številka) & 1 & 2 & 3 & 4 & 5 & 6 & 7 & 8 & 9 & 10 & 11 & 12 & 13 & 14 & 15
\end{tabular}

Sign for syntaxa (Oznaka sintaksonov)

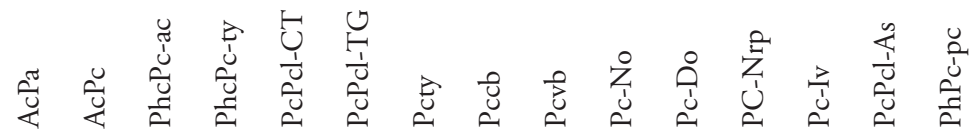

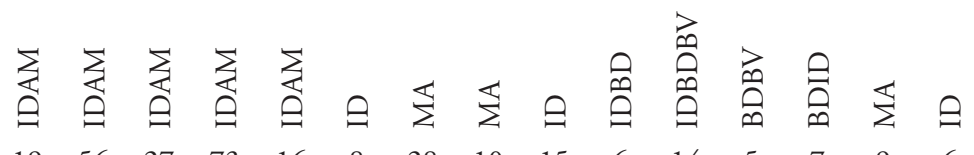

Author (Avtor)

\begin{tabular}{|c|c|c|c|c|c|c|c|c|c|c|c|c|c|c|c|c|}
\hline Pinguicula alpina & E1 & 100 & 86 & 41 & . & . & 50 & 7 & . & 60 & 33 & 14 & 40 & 71 & . & 17 \\
\hline Hymenostylium recurvirostre & E0 & 84 & 77 & 11 & 8 & 6 & . & . & . & 20 & . & 7 & . & . & . & . \\
\hline Palustriella commutata & E0 & 74 & 89 & 4 & 1 & . & . & . & . & 7 & . & . & . & . & . & \\
\hline Aster bellidiastrum & E1 & 58 & 43 & 85 & 45 & 19 & 88 & 7 & 20 & 93 & 83 & 43 & 60 & 100 & . & 50 \\
\hline Tofieldia calyculata & E1 & 58 & 54 & 37 & 16 & 6 & 38 & 4 & 40 & 20 & 17 & 14 & 60 & 43 & . & 33 \\
\hline Astrantia carniolica & E1 & 58 & 88 & 67 & . & . & 38 & . & . & 7 & . & . & . & . & . & 67 \\
\hline Orthothecium rufescens & E0 & 47 & 84 & 70 & 56 & 56 & 38 & 50 & 40 & 100 & 50 & 29 & . & . & . & 33 \\
\hline Paederota lutea & E1 & 26 & 39 & 100 & 74 & 94 & 100 & 100 & 100 & 20 & 67 & 29 & 80 & 86 & 100 & 83 \\
\hline Saxifraga aizoides & E1 & 21 & . & $\cdot$ & . & . & . & . & . & . & . & . & . & . & . & . \\
\hline Carex brachystachys & E1 & 16 & 32 & 48 & 45 & 50 & . & 4 & 50 & 47 & 33 & 7 & 20 & 43 & . & 33 \\
\hline Preissia quadrata & E0 & 16 & 29 & 4 & 12 & 19 & . & . & . & . & . & . & . & . & . & . \\
\hline Barbula crocea & E0 & 16 & 9 & 7 & 10 & 6 & . & . & . & . & . & . & . & . & . & . \\
\hline Pellia endiviifolia & E0 & 16 & 5 & 11 & 3 & 6 & . & . & . & . & . & 7 & . & . & . & . \\
\hline Valeriana saxatilis & E1 & 5 & 43 & 81 & 32 & 56 & 75 & 29 & 70 & 7 & . & . & . & . & 33 & 83 \\
\hline Fissidens dubius & E0 & 5 & 9 & 22 & 27 & 25 & 38 & 29 & 10 & 7 & . & 36 & 20 & . & . & \\
\hline Eucladium verticillatum & E0 & 5 & 7 & . & . & 6 & . & . & . & . & . & . & . & . & . & \\
\hline Selaginella helvetica & E1 & 5 & . & . & 3 & . & . & . & . & . & . & . & . & . & . & \\
\hline Primula carniolica & E1 & . & 100 & 100 & 100 & 100 & 100 & 100 & 100 & 100 & 100 & 100 & 100 & 100 & 100 & 100 \\
\hline Asplenium viride & E1 & . & 21 & 59 & 40 & 6 & 13 & 11 & 70 & 60 & 50 & 36 & 80 & 29 & . & . \\
\hline Valeriana tripteris & E1 & . & 11 & 37 & 49 & . & . & 18 & 30 & 53 & 83 & 64 & 80 & 71 & . & 17 \\
\hline Viola biflora & E1 & . & 7 & 93 & 16 & . & . & . & . & 100 & . & . & . & 14 & . & 67 \\
\hline Soldanella minima & E1 & . & 2 & 11 & . & . & . & . & . & . & . & . & . & . & . & 17 \\
\hline Moehringia muscosa & E1 & . & 2 & . & 8 & . & . & . & . & 7 & 33 & 36 & 20 & 29 & . & . \\
\hline Cystopteris fragilis & E1 & . & . & 26 & 12 & . & . & . & . & 53 & 33 & 14 & 60 & . & . & 17 \\
\hline Cystopteris regia & E1 & . & . & 11 & . & . & . & . & . & . & . & . & . & . & . & . \\
\hline Heliosperma pusillum & E1 & . & . & 4 & . & . & . & . & . & . & . & . & . & . & . & . \\
\hline Solorina saccata & E0 & . & . & 4 & . & . & . & 7 & . & . & . & . & . & . & . & . \\
\hline Primula $\times$ venusta & E1 & . & . & . & 4 & 19 & . & . & . & . & . & . & . & . & . & 17 \\
\hline Heliosperma veselskyi subsp. veselskyi & E1 & . & . & . & 1 & . & . & . & . & . & . & . & . & . & . & \\
\hline Collema fuscovirens & E0 & . & . & . & . & . & . & 21 & . & . & . & . & . & . & 56 & \\
\hline Ranunculus traunfellneri & E1 & . & . & . & . & . & . & . & . & . & . & . & . & . & . & 17 \\
\hline \multicolumn{17}{|c|}{ Physoplexido comosae-Saxifragion petraeae } \\
\hline Campanula cespitosa & E1 & 47 & 27 & 7 & 15 & 25 & . & . & 10 & 20 & . & 29 & 20 & . & . & . \\
\hline Phyteuma scheuchzeri subsp. columnae & E1 & 11 & 48 & 81 & 100 & 100 & 88 & . & . & 7 & . & . & . & . & . & 50 \\
\hline Micromeria thymifolia & E1 & 5 & . & . & . & . & . & . & . & . & . & . & . & . & . & . \\
\hline Hieracium pospichalii & E1 & . & 4 & . & . & . & . & . & . & . & . & . & 20 & . & . & . \\
\hline Athamanta turbith & E1 & . & . & . & 5 & 6 & 38 & . & . & . & . & . & . & . & . & . \\
\hline Saxifraga squarrosa & E1 & . & . & . & . & . & 25 & . & . & . & . & . & . & . & . & 33 \\
\hline Silene hayekiana & E1 & . & . & . & . & . & . & . & . & . & . & . & 20 & . & . & \\
\hline
\end{tabular}

Cystopteridion s. lat. (Astrantio carniolicae-Paederotion luteae nom. prov.) 


\begin{tabular}{|c|c|c|c|c|c|c|c|c|c|c|c|c|c|c|c|c|}
\hline Successive number (Zaporedna številka) & & 1 & 2 & 3 & 4 & 5 & 6 & 7 & 8 & 9 & 10 & 11 & 12 & 13 & 14 & 15 \\
\hline \multicolumn{17}{|l|}{ Potentilletalia caulescentis } \\
\hline Potentilla caulescens & E1 & 16 & 21 & 15 & 10 & 75 & 88 & 14 & . & . & . & . & 100 & . & 100 & . \\
\hline Kernera saxatilis & E1 & . & 4 & 4 & 7 & 19 & . & . & . & . & . & . & $\cdot$ & . & 22 & . \\
\hline Primula auricula & E1 & . & . & . & 1 & 25 & . & . & . & . & . & . & . & . & . & 17 \\
\hline Rhamnus pumilus & E1 & . & . & . & & 6 & 13 & . & 10 & . & . & 7 & . & 29 & 78 & 17 \\
\hline Campanula cochleariifolia & E1 & . & . & . & & 6 & 25 & . & . & . & . & . & . & . & . & 83 \\
\hline Asplenium seelosii & E1 & . & . & . & . & . & . & . & . & . & . & . & . & . & 33 & . \\
\hline Potentilla clusiana & E1 & . & . & . & . & . & . & . & . & . & . & . & . & . & . & 100 \\
\hline Bupleurum petraeum & E1 & . & . & . & . & . & . & . & . & . & . & . & . & . & . & 33 \\
\hline \multicolumn{17}{|l|}{ Asplenietea trichomanis } \\
\hline Asplenium trichomanes & E1 & 11 & 14 & 37 & 56 & 63 & 25 & 25 & 50 & 60 & 83 & 64 & 20 & 57 & 22 & . \\
\hline Asplenium ruta-muraria & E1 & . & 11 & 41 & 67 & 50 & 38 & 25 & 80 & 73 & 100 & 71 & 60 & 71 & 56 & . \\
\hline Hieracium glaucum & E1 & . & . & . & 3 & 13 & . & . & $\cdot$ & . & . & . & . & . & . & . \\
\hline Erysimum sylvestre & E1 & . & . & . & . & . & . & . & . & . & . & 7 & . & . & . & . \\
\hline \multicolumn{17}{|l|}{ Thlaspietea rotundifolii } \\
\hline Petasites paradoxus & E1 & 37 & 2 & . & . & . & . & . & . & . & . & . & . & . & . & . \\
\hline Achnatherum calamagrostis & E1 & 11 & 2 & . & . & . & . & . & . & . & . & . & . & . & . & . \\
\hline Adenostyles glabra & E1 & 11 & 13 & 33 & 3 & . & 25 & 4 & 10 & . & . & . & . & . & . & 17 \\
\hline Hieracium bifidum & E1 & 11 & 18 & 26 & 48 & 38 & 50 & . & 10 & 40 & 33 & 57 & 60 & 57 & . & 17 \\
\hline Hieracium porrifolium & E1 & 5 & 2 & . & 5 & 6 & 25 & . & . & . & . & 7 & . & . & . & . \\
\hline Gymnocarpium robertianum & E1 & . & 9 & 4 & 12 & 19 & . & . & . & 7 & 33 & 14 & 40 & . & . & . \\
\hline Aquilegia iulia & E1 & . & . & 4 & 1 & 6 & 13 & . & . & . & . & $\cdot$ & . & . & . & 50 \\
\hline Dryopteris villarii & E1 & . & . & 7 & 1 & . & $\cdot$ & . & . & . & . & . & . & . & . & $\cdot$ \\
\hline Saxifraga caesia & E1 & . & . & . & . & . & 13 & . & . & . & . & . & . & . & . & $\cdot$ \\
\hline Hladnikia pastinacifolia & E1 & . & . & . & . & . & $\cdot$ & . & . & . & . & . & . & . & . & 50 \\
\hline \multicolumn{17}{|l|}{ Caricetalia davallianae } \\
\hline Carex lepidocarpa & E1 & 21 & . & . & . & . & . & . & . & . & . & . & . & . & . & . \\
\hline Parnassia palustris & E1 & $\cdot$ & 5 & 4 & . & . & . & . & . & . & . & . & . & . & . & . \\
\hline Schoenus nigricans & E1 & . & 5 & . & . & . & . & . & . & . & . & . & . & . & . & . \\
\hline \multicolumn{17}{|l|}{ Montio-Cardaminetea } \\
\hline Conocephalum conicum & E0 & 11 & 32 & 48 & 25 & . & 13 & . & . & 80 & 67 & 14 & . & . & . & . \\
\hline Bryum pseudotriquetrum & E0 & 11 & 4 & . & . & 6 & . & . & . & . & . & $\cdot$ & . & . & . & . \\
\hline Cratoneuron filicinum & E0 & 5 & 4 & . & . & 6 & . & . & . & . & . & . & . & . & . & . \\
\hline Campylium stellatum & E0 & . & 7 & 4 & 1 & 13 & . & . & . & 13 & . & . & . & . & . & . \\
\hline Distichium capillaceum & E0 & . & 2 & 4 & 7 & . & . & . & . & . & . & . & . & . & . & 17 \\
\hline Ditrichum flexicaule & E0 & . & 2 & . & 1 & . & . & . & . & . & . & . & . & . & . & . \\
\hline Campylium calcareum & E0 & . & 2 & . & . & . & . & . & . & . & . & . & . & . & . & . \\
\hline Campylium elodes & E0 & . & 2 & . & . & . & . & . & . & . & . & . & . & . & . & . \\
\hline Cololejeunea calcarea & E0 & . & . & . & 1 & 6 & . & . & . & . & . & . & . & . & . & . \\
\hline Oxyrrhynchium hians & E0 & . & . & . & . & 6 & . & . & . & 20 & . & . & . & . & . & . \\
\hline Oxyrrhynchium schleicheri & E0 & . & . & . & . & . & . & . & . & 13 & . & . & . & . & . & . \\
\hline \multicolumn{17}{|l|}{ Elyno-Seslerietea } \\
\hline Sesleria caerulea & E1 & 68 & 61 & 59 & 53 & 94 & 100 & 75 & 100 & 20 & 33 & 29 & 40 & 43 & 89 & 100 \\
\hline Carex mucronata & E1 & 11 & 38 & 11 & 14 & 56 & 75 & 11 & 100 & . & . & . & 20 & . & 100 & 50 \\
\hline Phyteuma orbiculare & E1 & . & 9 & 4 & & 19 & . & 4 & 10 & . & 17 & . & . & 14 & . & . \\
\hline Carex ferruginea & E1 & . & 7 & 11 & 3 & . & 13 & . & . & . & . & . & . & . & . & . \\
\hline Carex firma & E1 & . & 4 & 7 & & 6 & 38 & . & . & . & . & . & . & . & . & 100 \\
\hline Betonica alopecuros & E1 & . & 2 & . & 5 & 25 & . & 7 & 10 & . & . & . & . & 14 & . & $\cdot$ \\
\hline Laserpitium peucedanoides & E1 & . & 2 & 15 & 1 & . & 38 & 11 & 10 & . & . & . & . & 29 & . & 50 \\
\hline Campanula witasekiana & E1 & . & . & 4 & . & . & . & . & $\cdot$ & . & . & . & . & . & . & . \\
\hline
\end{tabular}




\begin{tabular}{|c|c|c|c|c|c|c|c|c|c|c|c|c|c|c|c|c|}
\hline Successive number (Zaporedna številka) & & 1 & 2 & 3 & 4 & 5 & 6 & 7 & 8 & 9 & 10 & 11 & 12 & 13 & 14 & 15 \\
\hline Carduus crassifolius & E1 & . & . & . & 3 & . & . & . & . & . & . & . & . & . & . & . \\
\hline Gentiana clusii & E1 & . & . & . & 1 & 19 & . & . & . & . & $\cdot$ & . & . & . & . & 17 \\
\hline Hieracium villosum & E1 & . & . & . & & 13 & . & . & . & . & 17 & . & . & . & . & 17 \\
\hline Leontopodium alpinum & E1 & . & . & . & . & . & 13 & . & . & . & . & . & . & . & . & 17 \\
\hline Dryas octopetala & E1 & . & . & . & . & . & 13 & . & . & . & . & . & . & . & . & 17 \\
\hline Ranunculus carinthiacus? & E1 & . & . & . & . & . & 13 & . & . & . & . & . & . & . & . & . \\
\hline Hieracium pillosum & E1 & . & . & . & . & . & . & . & . & . & . & . & . & . & . & 50 \\
\hline \multicolumn{17}{|l|}{ Festuco-Brometea } \\
\hline Buphthalmum salicifolium & E1 & . & 4 & . & 3 & 6 & . & . & 10 & 13 & . & 7 & 20 & 29 & 11 & . \\
\hline Galium lucidum & E1 & . & 2 & . & 1 & . & . & . & 10 & . & . & 14 & . & . & . & . \\
\hline Carex humilis & E1 & . & . & . & 4 & 13 & . & 4 & 10 & . & . & $\cdot$ & . & . & . & . \\
\hline Brachypodium rupestre & E1 & . & . & . & 1 & . & . & . & $\cdot$ & . & . & . & . & . & . & . \\
\hline Linum catharticum & E1 & . & . & . & . & . & 13 & . & . & . & . & . & . & . & . & . \\
\hline Globularia punctata & E1 & . & . & . & . & . & . & 4 & . & . & . & . & . & . & . & . \\
\hline Campanula rotundifolia & E1 & . & . & . & . & . & . & . & 10 & . & . & . & . & . & . & . \\
\hline Sanguisorba minor & E1 & . & . & . & . & . & . & . & . & . & . & . & 20 & . & . & . \\
\hline \multicolumn{17}{|l|}{ Molinio-Arrbenatheretea, Calthion } \\
\hline Angelica sylvestris & E1 & 5 & . & . & 4 & . & . & . & . & . & . & . & . & . & . & . \\
\hline Pimpinella major & E1 & 5 & . & . & . & . & . & . & . & . & . & . & . & . & . & . \\
\hline Caltha palustris & E1 & . & 2 & . & 1 & . & . & . & . & . & . & . & . & . & . & . \\
\hline Cirsium oleraceum & E1 & . & 2 & . & . & . & . & . & . & . & . & . & . & 14 & . & . \\
\hline Taraxacum $\times$ Ruderalia & E1 & . & . & . & 1 & . & . & . & . & . & . & . & . & . & . & . \\
\hline Euphorbia villosa & E1 & . & . & . & . & . & . & . & . & . & . & . & . & 14 & . & . \\
\hline \multicolumn{17}{|l|}{ Trifolio-Geranietea } \\
\hline Laserpitium siler & E1 & . & 4 & . & 3 & 25 & . & . & . & . & . & 7 & . & 14 & 11 & . \\
\hline Laserpitium latifolium & E1 & . & 2 & . & 1 & . & . & . & . & . & . & 7 & 20 & 14 & . & . \\
\hline Digitalis grandiflora & E1 & . & . & 4 & . & . & . & . & . & . & . & . & . & 14 & . & . \\
\hline Viola hirta & E1 & . & . & . & 3 & 6 & . & . & 10 & . & 17 & . & . & . & . & . \\
\hline Anthericum ramosum & E1 & . & . & . & 1 & . & . & . & $\cdot$ & . & . & 7 & . & . & . & . \\
\hline Thalictrum minus & E1 & . & . & . & 1 & . & . & . & . & . & . & . & . & . & . & . \\
\hline Thesium bavarum & E1 & . & . & . & & 6 & . & . & . & . & . & . & . & . & . & . \\
\hline Vincetoxicum hirundinaria & E1 & . & . & . & . & . & . & . & . & . & . & 14 & . & . & 11 & . \\
\hline \multicolumn{17}{|l|}{ Betulo-Alnetea } \\
\hline Salix appendiculata & E1 & 21 & 13 & 7 & 7 & 44 & 25 & 4 & 10 & 13 & 17 & 21 & 40 & 43 & . & 33 \\
\hline Salix glabra & E1 & . & 11 & . & 3 & 13 & 63 & . & $\cdot$ & $\cdot$ & . & . & . & . & . & 33 \\
\hline \multicolumn{17}{|l|}{ Mulgedio-Aconitetea } \\
\hline Chaerophyllum hirsutum & E1 & 5 & 4 & 7 & & . & . & . & . & . & . & . & . & . & . & . \\
\hline Crepis paludosa & E1 & 5 & 2 & 4 & . & . & . & . & . & . & . & 14 & . & . & . & . \\
\hline Petasites hybridus & E1 & 5 & . & . & . & . & . & . & . & 7 & . & . & . & 14 & . & . \\
\hline Veratrum album & E1 & . & . & 4 & . & . & . & . & . & . & . & . & 20 & . & . & . \\
\hline Senecio ovatus & E1 & . & . & . & 5 & . & . & . & . & . & . & 7 & 40 & . & . & . \\
\hline Aconitum degenii subsp. paniculatum & E1 & . & . & . & 1 & . & . & . & . & . & 17 & 7 & . & . & . & . \\
\hline Ranunculus platanifolius & E1 & . & . & . & 1 & . & . & . & . & . & . & . & . & . & . & . \\
\hline Saxifraga rotundifolia & E1 & . & . & . & 1 & . & . & . & . & . & . & . & . & . & . & . \\
\hline Athyrium filix-femina & E1 & . & . & . & . & . & . & . & . & . & 17 & . & 20 & . & . & . \\
\hline Centaurea montana & E1 & . & . & . & . & . & . & . & . & . & . & . & 20 & . & . & . \\
\hline \multicolumn{17}{|l|}{ Epilobietea angustifolii } \\
\hline Eupatorium cannabinum & E1 & 5 & 5 & . & . & . & . & . & . & . & . & . & . & 14 & . & . \\
\hline Urtica dioica & E1 & . & . & . & . & . & . & . & . & . & 17 & . & . & . & . & . \\
\hline
\end{tabular}




\begin{tabular}{|c|c|c|c|c|c|c|c|c|c|c|c|c|c|c|c|c|}
\hline \multirow{2}{*}{\multicolumn{2}{|c|}{$\begin{array}{l}\text { Successive number (Zaporedna številka) } \\
\text { Sambuco-Salicion capreae, Rhamno-Prunetea }\end{array}$}} & 1 & 2 & 3 & 4 & 5 & 6 & 7 & 8 & 9 & 10 & 11 & 12 & 13 & 14 & 15 \\
\hline & & & & & & & & & & & & & & & & \\
\hline Rubus idaeus & E1 & . & . & 4 & 1 & . & . & . & . & . & . & . & . & . & . & . \\
\hline Juniperus communis (inc. J. intermedia) & $\mathrm{E} 2 \mathrm{a}$ & . & . & . & & 6 & . & . & 20 & . & . & 14 & . & . & . & . \\
\hline Salix caprea & E1 & . & . & . & . & . & . & . & . & . & . & . & 20 & . & . & . \\
\hline Sorbus aucuparia & E2a & . & . & . & . & . & . & . & . & . & . & . & 20 & . & . & . \\
\hline Viburnum lantana & E2a & . & . & . & . & . & . & . & . & . & . & . & . & 14 & . & . \\
\hline \multicolumn{17}{|l|}{ Erico-Pinetea } \\
\hline Molinia arundinacea & E1 & 58 & 43 & 4 & 1 & 6 & . & 4 & . & 7 & . & . & . & 29 & . & . \\
\hline Calamagrostis varia & E1 & 37 & 32 & 33 & 32 & 50 & 13 & . & . & 67 & 50 & 50 & . & 29 & . & . \\
\hline Erica carnea & E1 & 16 & 21 & 4 & 14 & 69 & 25 & 36 & 70 & 7 & 17 & 36 & 60 & . & 11 & . \\
\hline Carex ornithopoda & E1 & 11 & 5 & . & 5 & . & . & 11 & 20 & . & . & 29 & . & . & 11 & . \\
\hline Leontodon incanus & E1 & 11 & . & . & 1 & 19 & . & . & . & . & . & 7 & . & 14 & . & . \\
\hline Gymnadenia odoratissima & E1 & 5 & . & . & . & . & 13 & . & . & . & . & . & . & . & . & . \\
\hline Rhododendron hirsutum & E1 & . & 11 & 33 & 26 & 56 & 38 & 21 & 40 & 13 & . & 14 & 20 & 43 & . & 67 \\
\hline Rhodothamnus chamaecistus & E1 & . & 7 & 19 & 1 & 13 & 75 & . & . & . & . & . & . & . & . & 100 \\
\hline Aquilegia nigricans & E1 & . & 4 & 4 & 1 & 6 & . & . & . & . & . & 7 & 20 & . & . & . \\
\hline Cirsium erisithales & E1 & . & 2 & 4 & 3 & . & . & . & . & . & . & . & . & 14 & . & . \\
\hline Polygala chamaebuxus & E1 & . & 2 & 4 & 5 & 50 & 13 & 7 & 30 & . & . & . & . & . & 22 & . \\
\hline Pinus nigra & E1 & . & . & 4 & & 6 & . & . & 10 & . & . & . & . & . & . & 17 \\
\hline Rubus saxatilis & E1 & . & . & . & 5 & . & . & . & . & . & . & . & . & 29 & . & . \\
\hline Carex alba & E1 & . & . & . & 4 & . & . & 7 & . & . & 17 & 29 & . & . & . & . \\
\hline Amelanchier ovalis & E1 & . & . & . & 1 & 6 & 25 & . & 40 & . & . & . & 20 & $\cdot$ & 11 & . \\
\hline Pinus sylvestris & E2a & . & . & . & 1 & . & . & . & . & . & . & . & . & 14 & . & . \\
\hline Aster amellus & E1 & . & . & . & 1 & . & $\cdot$ & . & . & . & . & . & . & . & . & . \\
\hline Asperula aristata & E1 & . & . & . & & 19 & 13 & . & $\cdot$ & . & $\cdot$ & . & . & . & . & . \\
\hline Globularia cordifolia & E1 & . & . & . & & 6 & 13 & 4 & 10 & . & 17 & . & . & . & 11 & . \\
\hline Genista januensis & E1 & . & . & . & & 6 & . & . & $\cdot$ & . & $\cdot$ & . & . & . & . & . \\
\hline Allium ericetorum & E1 & . & . & . & . & . & . & . & 10 & . & . & . & . & . & . & . \\
\hline Cotoneaster tomentosus & E1 & . & . & . & . & . & . & . & 10 & . & . & . & . & . & . & . \\
\hline Chamaecytisus hirsutus & E1 & . & . & . & . & . & . & . & $\cdot$ & . & . & 7 & . & . & . & . \\
\hline Euphrasia cuspidata? & E1 & . & . & . & . & . & . & . & . & . & . & . & . & . & 22 & . \\
\hline \multicolumn{17}{|l|}{ Vaccinio-Piceetea } \\
\hline Picea abies & E1 & 5 & 2 & . & 3 & . & 13 & 18 & . & . & 17 & 21 & 40 & 71 & . & . \\
\hline Veronica urticifolia & E1 & 5 & 7 & 4 & 18 & 6 & . & . & . & 73 & 50 & . & . & . & . & . \\
\hline Solidago virgaurea & E1 & . & 4 & . & 4 & . & . & . & . & . & . & . & . & . & . & . \\
\hline Aposeris foetida & E1 & . & 2 & . & 1 & . & . & . & . & 7 & . & . & 20 & . & . & . \\
\hline Gentiana asclepiadea & E1 & . & 2 & . & 3 & . & . & 4 & $\cdot$ & . & . & . & . & . & . & . \\
\hline Homogyne sylvestris & E1 & . & 2 & 15 & 15 & . & . & 21 & 40 & 13 & 17 & 14 & 60 & . & . & . \\
\hline Oxalis acetosella & E1 & . & 2 & . & 3 & . & . & . & . & 13 & 17 & . & . & . & . & . \\
\hline Clematis alpina & E1 & . & . & 15 & 4 & 13 & . & . & $\cdot$ & 7 & 17 & . & . & . & . & . \\
\hline Hieracium murorum & E1 & . & . & . & 4 & 13 & . & . & 10 & . & 50 & . & 20 & . & . & . \\
\hline Polystichum lonchitis & E1 & . & . & . & 1 & . & . & . & . & . & . & . & . & . & . & . \\
\hline Rosa pendulina & E1 & . & . & . & 1 & . & . & 4 & 10 & . & . & 7 & . & . & . & . \\
\hline Abies alba & E1 & . & . & . & . & . & . & 4 & 30 & . & . & 14 & 20 & . & . & . \\
\hline Rhytidiadelphus triquetrus & E0 & . & . & . & . & . & . & . & . & . & . & . & 40 & . & . & . \\
\hline \multicolumn{17}{|l|}{ Aremonio-Fagion } \\
\hline Cyclamen purpurascens & E1 & . & 13 & 7 & 23 & 19 & . & 14 & 60 & 13 & 33 & 7 & 20 & . & . & . \\
\hline Euphorbia carniolica & E1 & . & 9 & . & 3 & . & . & . & $\cdot$ & 7 & . & . & 20 & . & . & . \\
\hline Primula vulgaris & E1 & . & 5 & . & 4 & 6 & . & 7 & . & . & . & . & $\cdot$ & . & . & . \\
\hline
\end{tabular}




\begin{tabular}{|c|c|c|c|c|c|c|c|c|c|c|c|c|c|c|c|c|}
\hline Successive number (Zaporedna številka) & & 1 & 2 & 3 & 4 & 5 & 6 & 7 & 8 & 9 & 10 & 11 & 12 & 13 & 14 & 15 \\
\hline Cardamine trifolia & E1 & . & 4 & 4 & 4 & . & . & . & . & 7 & . & . & 20 & . & . & . \\
\hline Helleborus niger & E1 & . & 4 & . & 7 & 6 & 13 & 14 & 40 & 7 & 33 & 21 & . & 14 & . & . \\
\hline Knautia drymeia & E1 & . & 4 & . & 1 & . & . & . & . & . & . & . & . & 14 & . & . \\
\hline Potentilla carniolica & E1 & . & 4 & . & 4 & . & . & . & . & . & . & . & . & . & . & . \\
\hline Anemone trifolia & E1 & . & 2 & 7 & 4 & . & 13 & . & . & . & . & . & . & . & . & . \\
\hline Hemerocallis lilioasphodelus & E1 & . & 2 & . & 4 & . & 25 & . & . & . & . & 7 & . & . & . & . \\
\hline Lamium orvala & E1 & . & 2 & . & 1 & . & . & . & . & . & . & . & . & . & . & . \\
\hline Dentaria enneaphyllos & E1 & . & . & 4 & & . & . & . & . & 7 & 33 & . & . & . & . & . \\
\hline Laserpitium krapfii & E1 & . & . & . & 1 & . & . & . & 10 & . & . & . & . & . & . & . \\
\hline Rhamnus fallax & E1 & . & . & . & 1 & . & . & . & 10 & . & . & . & . & . & . & . \\
\hline Omphalodes verna & E1 & . & . & . & 1 & . & . & . & . & . & 17 & . & . & 14 & . & . \\
\hline Scopolia carniolica & E1 & . & . & . & . & . & . & . & . & 13 & 33 & 7 & 20 & . & . & . \\
\hline \multicolumn{17}{|l|}{ Tilio-Acerion } \\
\hline Aruncus dioicus & E1 & . & 4 & 4 & 11 & . & . & . & . & 33 & 33 & 14 & 20 & 14 & . & . \\
\hline Phyllitis scolopendrium & E1 & . & 4 & . & 8 & . & . & . & . & . & 33 & . & . & . & . & . \\
\hline Acer pseudoplatanus & E1 & . & 2 & 7 & 7 & . & . & 11 & . & 7 & . & 14 & 20 & . & . & . \\
\hline Polystichum aculeatum & E1 & . & 2 & . & 8 & . & . & . & . & 13 & 33 & 7 & 40 & . & . & . \\
\hline Thalictrum aquilegiifolium & E1 & . & . & 4 & 4 & . & . & 7 & 20 & 27 & . & 21 & 40 & . & . & . \\
\hline Geranium robertianum & E1 & . & . & . & 5 & . & . & . & $\cdot$ & . & . & 21 & 20 & 29 & . & . \\
\hline Tephroseris pseudocrispa & E1 & . & . & . & 4 & . & . & . & . & . & . & . & . & . & . & . \\
\hline Acer platanoides & E2a & . & . & . & 1 & . & . & . & . & . & . & . & . & . & . & . \\
\hline Ulmus glabra & $\mathrm{E} 2 \mathrm{a}$ & . & . & . & 1 & . & . & . & . & 13 & . & . & . & . & . & . \\
\hline \multicolumn{17}{|l|}{ Fagetalia sylvaticae } \\
\hline Galium laevigatum & E1 & 11 & 4 & 7 & 7 & . & . & 7 & . & 13 & . & 14 & 20 & . & . & . \\
\hline Fagus sylvatica & E1 & 5 & . & 7 & 7 & 13 & 13 & 4 & 20 & 7 & 17 & 14 & 60 & 29 & . & . \\
\hline Laburnum alpinum & E2a & 5 & . & . & . & . & 25 & . & . & . & . & . & . & . & . & . \\
\hline Salvia glutinosa & E1 & 5 & 9 & 4 & 14 & . & . & 7 & . & . & . & . & 20 & . & . & . \\
\hline Mycelis muralis & E1 & . & 20 & 7 & 8 & 6 & . & 4 & . & 7 & 50 & 14 & . & . & . & . \\
\hline Galeobdolon flavidum & E1 & . & 5 & 11 & 11 & . & 13 & . & . & 47 & . & . & 20 & . & . & . \\
\hline Brachypodium sylvaticum & E1 & . & 4 & . & . & . & . & . & . & . & . & $\cdot$ & $\cdot$ & . & . & . \\
\hline Melica nutans & E1 & . & 4 & . & 3 & . & . & . & . & . & . & 29 & 20 & . & . & . \\
\hline Asarum europaeum subsp. caucasicum & E1 & . & 2 & . & 1 & . & . & . & . & . & . & . & . & . & . & . \\
\hline Dentaria pentaphyllos & E1 & . & 2 & . & 1 & . & . & . & . & 27 & 50 & . & . & . & . & . \\
\hline Prenanthes purpurea & E1 & . & 2 & . & . & . & . & . & . & 7 & . & 7 & 40 & . & . & . \\
\hline Mercurialis perennis & E1 & . & . & . & 10 & . & . & 7 & 30 & 7 & . & 21 & 20 & 14 & . & . \\
\hline Symphytum tuberosum & E1 & . & . & . & 3 & . & . & . & . & . & . & . & . & . & . & . \\
\hline Campanula trachelium & E1 & . & . & . & 1 & . & . & . & . & 20 & 67 & 7 & . & . & . & . \\
\hline Lathyrus vernus & E1 & . & . & . & 1 & . & . & . & . & 13 & . & . & . & . & . & . \\
\hline Petasites albus & E1 & . & . & . & 1 & . & . & . & . & . & . & . & 20 & . & . & . \\
\hline Sambucus nigra & $\mathrm{E} 2 \mathrm{a}$ & . & . & . & 1 & . & . & . & . & . & . & . & . & . & . & . \\
\hline Viola reichenbachiana & E1 & . & . & . & . & . & . & 4 & . & . & . & . & . & . & . & . \\
\hline Daphne mezereum & E2a & . & . & . & . & . & . & . & 10 & 7 & . & . & 20 & . & . & . \\
\hline Lonicera alpigena & $\mathrm{E} 2 \mathrm{a}$ & . & . & . & . & . & . & . & $\cdot$ & . & 17 & . & $\cdot$ & . & . & . \\
\hline Myosotis sylvatica agg. & E1 & . & . & . & . & . & . & . & . & . & 33 & . & . & . & . & . \\
\hline Heracleum sphondylium & E1 & . & . & . & . & . & . & . & . & . & . & 7 & . & . & . & . \\
\hline Poa nemoralis & E1 & . & . & . & . & . & . & . & . & . & . & . & 20 & . & . & . \\
\hline Polygonatum multiflorum & E1 & . & . & . & . & . & . & . & . & . & . & . & 20 & . & . & . \\
\hline \multicolumn{17}{|l|}{ Quercetalia pubescenti-petraeae } \\
\hline Carex flacca & E1 & 21 & 5 & . & & . & . & . & . & . & . & . & . & 14 & . & . \\
\hline Ostrya carpinifolia & E1 & 11 & 2 & 4 & 10 & 50 & 13 & 21 & 60 & . & . & 14 & . & 29 & 33 & . \\
\hline
\end{tabular}




\begin{tabular}{|c|c|c|c|c|c|c|c|c|c|c|c|c|c|c|c|c|}
\hline Successive number (Zaporedna številka) & & 1 & 2 & 3 & 4 & 5 & 6 & 7 & 8 & 9 & 10 & 11 & 12 & 13 & 14 & 15 \\
\hline Fraxinus ornus & E1 & . & 5 & 7 & 15 & 38 & 38 & 14 & 20 & . & . & 21 & 20 & . & 18 & . \\
\hline Mercurialis ovata & E1 & . & . & . & 7 & 25 & . & . & . & . & . & . & . & . & . & . \\
\hline Clematis recta & E1 & . & . & . & 1 & . & . & . & . & . & . & . & . & . & . & . \\
\hline Euonymus verrucosa & $\mathrm{E} 2 \mathrm{a}$ & . & . & . & 1 & . & . & . & . & . & . & . & . & . & . & . \\
\hline Melittis melissophyllum & E1 & . & . & . & & 6 & . & . & . & . & . & . & . & $\cdot$ & . & . \\
\hline Sorbus aria & $\mathrm{E} 2 \mathrm{a}$ & . & . & . & . & . & . & . & 30 & . & . & 7 & . & 29 & . & . \\
\hline \multicolumn{17}{|l|}{ Querco-Fagetea } \\
\hline Potentilla erecta & E1 & 5 & . & . & . & . & . & . & . & . & . & . & . & 14 & . & . \\
\hline Carex digitata & E1 & . & 32 & 33 & 38 & 19 & 13 & . & . & 60 & 33 & 21 & 40 & . & . & . \\
\hline Hedera helix & E1 & . & 9 & . & 8 & 6 & . & . & . & . & . & 7 & . & . & . & . \\
\hline Clematis vitalba & E1 & . & 7 & . & 1 & . & . & . & . & . & . & . & . & . & . & . \\
\hline Carex umbrosa & E1 & . & 5 & 4 & . & . & . & . & . & . & . & . & . & . & . & . \\
\hline Hepatica nobilis & E1 & . & 5 & 7 & 22 & 6 & . & 11 & 10 & 33 & 33 & 7 & 20 & . & . & . \\
\hline Frangula alnus & E2a & . & 2 & . & $\cdot$ & . & . & $\cdot$ & $\cdot$ & $\cdot$ & $\cdot$ & . & $\cdot$ & . & . & . \\
\hline Taxus baccata & E1 & . & 2 & . & . & . & . & . & . & 7 & . & 7 & . & . & . & . \\
\hline Veratrum nigrum & E1 & . & . & . & 5 & . & . & . & . & . & $\cdot$ & . & . & . & . & $\cdot$ \\
\hline Acer campestre & E1 & . & . & . & 1 & . & . & . & . & . & . & . & . & . & . & . \\
\hline Cardamine impatiens & E1 & . & . & . & 1 & . & . & . & . & . & . & . & . & . & . & . \\
\hline Lonicera xylosteum & E1 & . & . & . & 1 & . & . & . & . & . & . & . & . & . & . & . \\
\hline Quercus petraea & E1 & . & . & . & 1 & . & . & . & . & . & . & . & $\cdot$ & . & . & . \\
\hline Anemone nemorosa & E1 & . & . & . & . & . & . & . & . & . & 17 & 7 & 20 & . & . & . \\
\hline Corylus avellana & E1 & . & . & . & . & . & . & . & . & . & . & . & 20 & . & . & . \\
\hline \multicolumn{17}{|l|}{ Salicetea purpureae } \\
\hline Salix eleagnos & E2a & 21 & . & . & . & . & . & . & . & . & . & . & . & . & . & . \\
\hline \multicolumn{17}{|l|}{ Mosses and lichens (Mahovi in lišaji) } \\
\hline Brachythecium rutabulum & E0 & 5 & 2 & 11 & 1 & . & . & $\cdot$ & $\cdot$ & . & . & $\cdot$ & $\cdot$ & . & . & $\cdot$ \\
\hline Ctenidium molluscum & E0 & 5 & 14 & 52 & 58 & 50 & 25 & 21 & 60 & 33 & 83 & 64 & 40 & . & . & 33 \\
\hline Neckera crispa & E0 & 5 & 27 & 41 & 73 & 25 & 38 & 54 & 70 & 40 & 100 & 71 & 100 & . & 22 & 50 \\
\hline Tortella tortuosa & E0 & 5 & 11 & 33 & 40 & 38 & 25 & 57 & 50 & 20 & 33 & 7 & $\cdot$ & . & $\cdot$ & 83 \\
\hline Leiocolea collaris & E0 & 5 & . & $\cdot$ & 3 & . & . & . & . & . & $\cdot$ & . & . & . & . & . \\
\hline Reboulia hemisphaerica & E0 & 5 & . & . & . & . & . & . & . & . & . & . & $\cdot$ & . & . & . \\
\hline Marchantia polymorpha & E0 & . & 4 & 4 & 3 & . & . & 4 & . & 7 & . & . & 40 & . & . & . \\
\hline Heterocladium heteropterum & E0 & . & 4 & . & . & . & . & . & . & . & . & . & $\cdot$ & . & . & . \\
\hline Mnium thomsonii & E0 & . & 4 & . & 8 & . & . & . & . & 27 & . & . & . & . & . & . \\
\hline Bryum sp. & E0 & . & 2 & 4 & . & . & . & . & . & . & . & . & . & . & . & . \\
\hline Mnium marginatum & E0 & . & 2 & . & 1 & . & . & . & . & 20 & . & . & . & . & . & . \\
\hline Dichodontium pellucidum & E0 & . & 2 & . & . & . & . & . & . & . & . & . & . & . & . & . \\
\hline Encalypta vulgaris & E0 & . & 2 & . & . & . & . & . & . & . & . & . & . & . & . & . \\
\hline Lophozia sp. & E0 & . & 2 & . & 1 & . & . & . & . & . & . & . & . & . & . & . \\
\hline Rhynchostegium murale & E0 & . & 2 & . & . & . & . & . & . & . & . & . & . & . & . & . \\
\hline Schistidium apocarpum & E0 & . & . & 4 & 5 & 6 & . & . & 30 & . & . & . & . & . & 33 & . \\
\hline Pedinophyllum interruptum & E0 & . & . & 4 & 3 & 6 & . & . & $\cdot$ & 20 & . & . & . & . & $\cdot$ & . \\
\hline Plagiochila porelloides & E0 & . & . & 4 & 4 & . & . & . & . & $\cdot$ & . & . & . & . & $\cdot$ & . \\
\hline Plagiopus oederi & E0 & . & . & 7 & . & . & . & . & . & . & . & . & . & . & . & . \\
\hline Lescuraea plicata & E0 & . & . & 4 & . & . & . & . & . & . & . & . & . & . & . & . \\
\hline Dicranum scoparium & E0 & . & . & 4 & . & . & . & . & . & . & . & . & . & . & . & . \\
\hline Encalypta streptocarpa & E0 & . & . & . & 4 & 6 & . & 7 & . & . & . & . & . & . & . & . \\
\hline Isothecium alopecuroides & E0 & . & . & . & 3 & . & . & . & . & . & . & . & . & . & . & . \\
\hline Mnium stellare & E0 & . & . & . & 3 & . & . & . & . & . & . & 7 & . & . & . & . \\
\hline Bryum capillare & E0 & . & . & . & 3 & . & . & . & . & . & . & . & . & . & . & . \\
\hline
\end{tabular}




\begin{tabular}{|c|c|c|c|c|c|c|c|c|c|c|c|c|c|c|c|c|}
\hline Successive number (Zaporedna številka) & & 1 & 2 & 3 & 4 & 5 & 6 & 7 & 8 & 9 & 10 & 11 & 12 & 13 & 14 & 15 \\
\hline Plagiomnium rostratum & E0 & . & . & . & 1 & 6 & . & . & . & . & . & . & . & . & . & . \\
\hline Dichodontium pellucidum subsp. flavescens & E0 & . & . & . & 1 & . & . & . & . & . & . & . & . & . & . & . \\
\hline Didymodon ferrugineus & E0 & . & . & . & 1 & . & . & . & . & . & . & . & . & . & . & . \\
\hline Plagiobryum zierii & E0 & . & . & . & 1 & . & . & . & . & . & . & . & . & . & . & . \\
\hline Lejeunea cavifolia & E0 & . & . & . & 1 & . & . & . & . & . & . & . & . & . & . & . \\
\hline Myurella sibirica & E0 & . & . & . & 1 & . & . & . & . & . & . & . & . & . & . & . \\
\hline Neckera complanata & E0 & . & . & . & 1 & 6 & . & . & . & . & . & . & . & . & . & . \\
\hline Trichostomum brachydontium & E0 & . & . & . & 1 & . & . & . & . & . & . & . & . & . & . & . \\
\hline Myurella julacea & E0 & . & . & . & 1 & . & . & . & . & . & . & . & . & . & . & . \\
\hline Lichenes div. & E0 & . & . & . & 1 & 6 & 13 & . & . & . & . & . & . & . & . & . \\
\hline Homalothecium sericeum & E0 & . & . & . & . & 6 & . & . & . & . & . & 7 & . & . & 33 & . \\
\hline Dicranodontium denudatum & E0 & . & . & . & . & 6 & . & . & . & . & . & . & . & . & . & . \\
\hline Trichostomum crispulum & E0 & . & . & . & . & 6 & . & . & . & . & . & . & . & . & . & . \\
\hline Anomodon attenuatus & E0 & . & . & . & . & 6 & . & . & . & . & . & . & . & . & . & . \\
\hline Anomodon viticulosus & E0 & . & . & . & . & 6 & . & . & . & . & . & . & . & . & . & . \\
\hline Musci sp. & E0 & . & . & . & . & . & 13 & . & $\cdot$ & . & . & . & . & . & $\cdot$ & . \\
\hline Lepraria crassissima & E0 & . & . & . & . & . & . & 32 & 70 & . & . & . & . & . & 22 & . \\
\hline Collema polycarpon & E0 & . & . & . & . & . & . & 18 & . & . & . & . & . & . & . & . \\
\hline Trentepoblia aurea & E0 & . & . & . & . & . & . & 4 & 10 & . & . & . & . & . & . & . \\
\hline Brachythecium sp. & E0 & . & . & . & . & . & . & . & 10 & . & . & . & . & . & . & . \\
\hline Brachythecium starkei & E0 & . & . & . & . & . & . & . & . & 7 & . & . & . & . & . & . \\
\hline Cirriphyllum cirrhosum & E0 & . & . & . & . & . & . & . & . & 7 & . & . & . & . & . & . \\
\hline Plagiomnium undulatum & E0 & . & . & . & . & . & . & . & . & . & 17 & . & 60 & . & $\cdot$ & . \\
\hline Tortella sp. & E0 & . & . & . & . & . & & . & . & . & . & . & . & . & 33 & . \\
\hline Caloplaca sp. & E0 & . & . & . & . & . & . & . & . & . & . & . & . & . & 22 & . \\
\hline
\end{tabular}

\section{Legend (Legenda)}

1 AcPa Astrantio carniolicae-Pinguiculetum alpinae, this article, Table 2.

2 AcPc Astrantio carniolicae-Primuletum carniolicae, this article, Table 1.

3 PhcPc-ac Phyteumato columnae-Primuletum carniolicae astrantietosum carniolicae, this article, Table 6.

4 PhcPc-ty Phyteumato columnae-Primuletum carniolicae typicum, this article, Table 5.

5 PcPcl-CT Phyteumato columnae-Primuletum carniolicae potentilletosum caulescentis, this article, Table 7.

6 PcPcl-TG Phyteumato columnae-Primuletum carniolicae potentilletosum carniolicae (Primulo carniolicae-Potentilletum caulescentis), Dakskobler (1998, Table 1, pp. 286-287).

7 Pcty Primuletum carniolicae, Accetto (2008, Table 1, pp. 42-44).

8 Pccb Primuletum carniolicae caricetosum brachystachyos, Accetto (2015, Table 27, pp. 98-99).

9 Pcvb Primuletum carniolicae violetosum biflorae, this article, Table 4.

10 Pc-No Primuletum carniolicae s. lat., Dolinar et al. (2017, Table 1, pp. 30-32).

11 Pc-Do Primuletum carniolicae s. lat., Dolinar et al. (2013, Table 1, pp. 13-16).

12 Pc-Npr Primuletum carniolicae s. lat., Dolinar et al. (2015, Table 1, pp. 40-43).

13 Pc-Iv Primuletum carniolicae s. lat., Dolinar et al. (2013: Table 2, pp. 17-18).

14 PcPcl-As Paederoto luteae-Potentilletum caulescentis (Primulo carniolicae-Potentilletum caulescentis var. Asplenium seelosii), Accetto (2008, Table 5, pp. 49-50).

15 PcPcl Primulo carniolicae-Potentilletum clusianae, this article, Table 8.

ID Igor Dakskobler

AM Andrej Martinčič

BD Branko Dolinar
BV Branko Vreš

MA Marko Accetto 


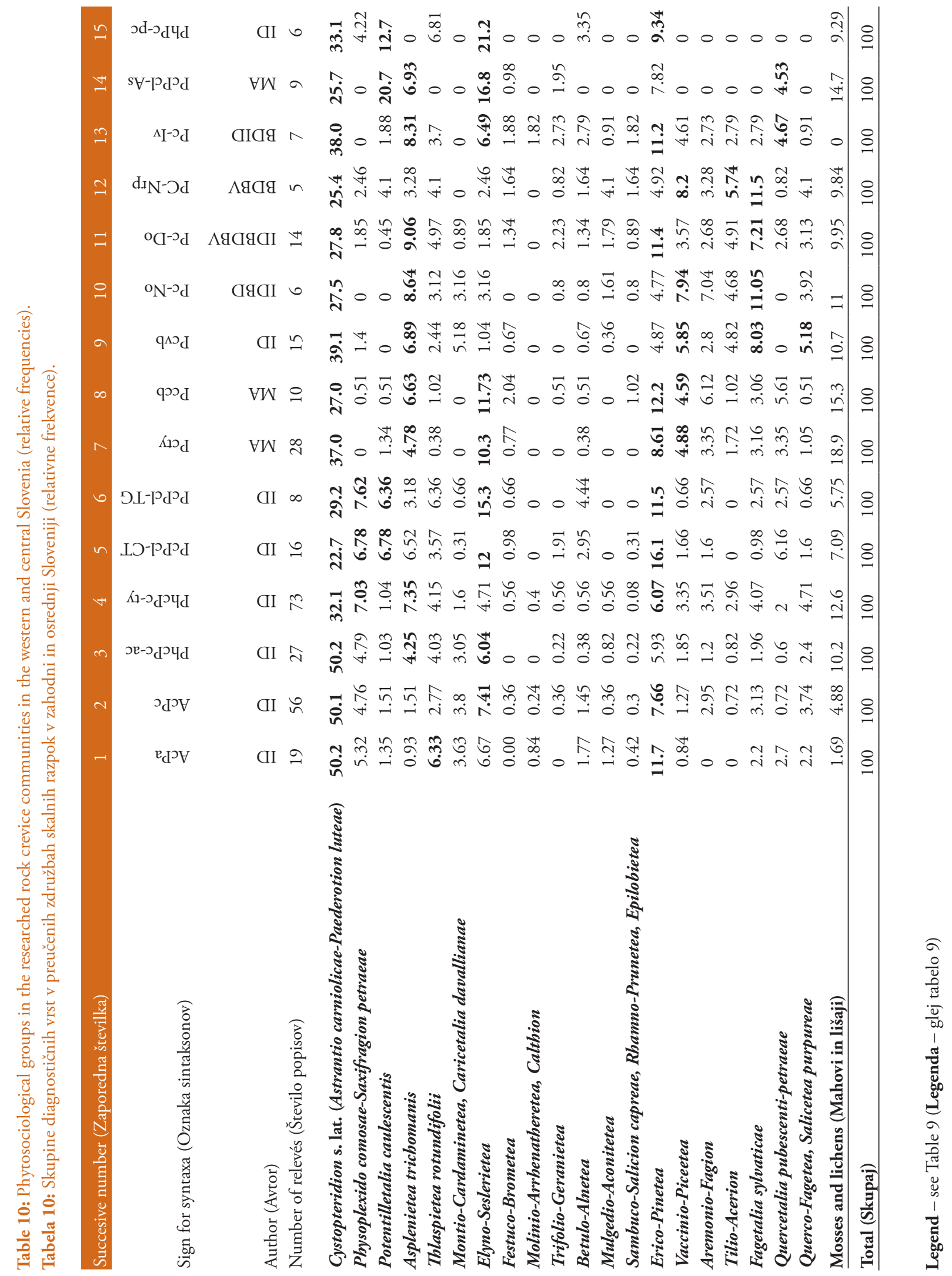


Table 11: List of species in rock crevice communities with Primula carniolica, stored in the FLoVegSi database, with frequency more than $10 \%$.

Tabela 11: Seznam vrst v združbah skalnih razpok z vrsto Primula carniolica s frekvenco več kot $10 \%$.

\begin{tabular}{|c|c|c|}
\hline & $\begin{array}{l}\text { Number } \\
\text { of relevés }\end{array}$ & Frequency \\
\hline \multicolumn{3}{|l|}{ Vascular plants (Cevnice) } \\
\hline Primula carniolica & 348 & 100 \\
\hline Paederota lutea & 209 & 60 \\
\hline Phyteuma scheuchzeri subsp. columnae & 196 & 56 \\
\hline Sesleria caerulea & 187 & 54 \\
\hline Aster bellidiastrum & 178 & 51 \\
\hline Astrantia carniolica & 147 & 42 \\
\hline Asplenium ruta-muraria & 147 & 42 \\
\hline Valeriana saxatilis & 142 & 41 \\
\hline Asplenium trichomanes & 141 & 41 \\
\hline Calamagrostis varia & 139 & 40 \\
\hline Carex brachystachys & 132 & 38 \\
\hline Carex digitata & 126 & 36 \\
\hline Pinguicula alpina & 120 & 34 \\
\hline Valeriana tripteris & 118 & 34 \\
\hline Asplenium viride & 114 & 33 \\
\hline Tofieldia calyculata & 109 & 31 \\
\hline Hieracium bifidum & 105 & 30 \\
\hline Carex mucronata & 77 & 22 \\
\hline Rhododendron hirsutum & 76 & 22 \\
\hline Erica carnea & 75 & 22 \\
\hline Cyclamen purpurascens & 63 & 18 \\
\hline Viola biflora & 60 & 17 \\
\hline Campanula cespitosa & 55 & 16 \\
\hline Veronica urticifolia & 55 & 16 \\
\hline Potentilla caulescens & 52 & 15 \\
\hline Salix appendiculata & 47 & 14 \\
\hline Molinia arundinacea & 45 & 13 \\
\hline Galeobdolon flavidum & 44 & 13 \\
\hline Hepatica nobilis & 44 & 13 \\
\hline Ostrya carpinifolia & 43 & 12 \\
\hline Cystopteris fragilis & 41 & 12 \\
\hline Fraxinus ornus & 39 & 11 \\
\hline Aruncus dioicus & 37 & 11 \\
\hline Salvia glutinosa & 35 & 10 \\
\hline \multicolumn{3}{|l|}{ Mosses (Mahovi) } \\
\hline Orthothecium rufescens & 221 & 64 \\
\hline Neckera crispa & 166 & 48 \\
\hline Ctenidium molluscum & 143 & 41 \\
\hline Conocephalum conicum & 110 & 32 \\
\hline Tortella tortuosa & 101 & 29 \\
\hline Palustriella commutata & 62 & 18 \\
\hline Fissidens dubius & 56 & 16 \\
\hline Hymenostylium recurvirostre & 48 & 14 \\
\hline
\end{tabular}

